TEXTURE CORRELATION FEATURE FOR

SUPPORT VECTOR MACHINE-BASED FACE DETECTION

\author{
A Thesis \\ Presented to \\ The Faculty of Graduate Studies \\ of \\ The University of Guelph
}

\begin{abstract}
by
NGUYEN LE
In partial fulfilment of requirements
for the degree of
Master of Science

October, 2009

(C)Nguyen Le, 2009 


$\begin{array}{ll}\begin{array}{l}\text { Library and Archives } \\ \text { Canada }\end{array} & \begin{array}{l}\text { Bibliothèque et } \\ \text { Archives Canada }\end{array} \\ \begin{array}{l}\text { Published Heritage } \\ \text { Branch }\end{array} & \begin{array}{l}\text { Direction du } \\ \text { Patrimoine de l'édition }\end{array} \\ \begin{array}{l}\text { 395 Wellington Street } \\ \text { Ottawa ON K1A ON4 } \\ \text { Canada }\end{array} & \begin{array}{l}395, \text { rue Wellington } \\ \text { Ottawa ON K1A ON4 } \\ \text { Canada }\end{array}\end{array}$

Your file Votre néférence

ISBN: 978-0-494-58422-4

Our file Notre référence

ISBN: $978-0-494-58422-4$

NOTICE:

The author has granted a nonexclusive license allowing Library and Archives Canada to reproduce, publish, archive, preserve, conserve, communicate to the public by telecommunication or on the Internet, loan, distribute and sell theses worldwide, for commercial or noncommercial purposes, in microform, paper, electronic and/or any other formats.

The author retains copyright ownership and moral rights in this thesis. Neither the thesis nor substantial extracts from it may be printed or otherwise reproduced without the author's permission.
AVIS:

L'auteur a accordé une licence non exclusive permettant à la Bibliothèque et Archives Canada de reproduire, publier, archiver, sauvegarder, conserver, transmettre au public par télécommunication ou par l'Internet, prêter, distribuer et vendre des thèses partout dans le monde, à des fins commerciales ou autres, sur support microforme, papier, électronique et/ou autres formats.

L'auteur conserve la propriété du droit d'auteur et des droits moraux qui protège cette thèse. $\mathrm{Ni}$ la thèse ni des extraits substantiels de celle-ci ne doivent être imprimés ou autrement reproduits sans son autorisation.
In compliance with the Canadian Privacy Act some supporting forms may have been removed from this thesis.

While these forms may be included in the document page count, their removal does not represent any loss of content from the thesis.
Conformément à la loi canadienne sur la protection de la vie privée, quelques formulaires secondaires ont été enlevés de cette thèse.

Bien que ces formulaires aient inclus dans la pagination, il r'y aura aucun contenu manquant.

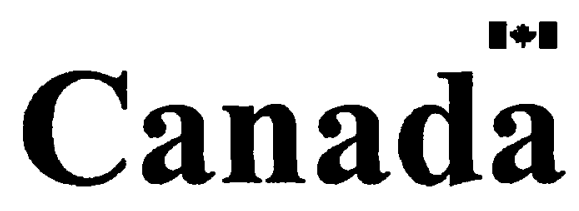




\section{ABSTRACT \\ TEXTURE CORRELATION FEATURE FOR SUPPORT VECTOR MACHINE-BASED FACE DETECTION}

Nguyen Le

University of Guelph, 2009
Advisor:

Dr. Deb Stacey and Alex Ferworn

Human face detection is an important step in any human face processing applications such as human face recognition systems, surveillance systems, face tracking, and expression recognition, et cetera. The problems associated with face detection is the fact that human face is a non-rigid object which is characterized by large variations. Therefore, it is difficult to model: Despite all the past efforts, many face detection methods are still too inflexible to be applicable for real world applications. In this thesis, our goal is to improve previous appearance-based face detection work. We examine the use of the texture correlation feature for appearance-based face detection. It turns out that our system with the texture correlation feature can help to address some shortcomings of systems using the grey scale feature. Our system consists of two parts. The feature extraction part is responsible for extracting texture correlation features. The second part is a Support Vector Machine which performs the classification task. 


\section{Acknowledgements}

Writing this thesis has been a wonderful journey for me. I would like to take this opportunity to thank all the people has helped me to make this possible.

I would like to thank my supervisors, Dr. Deborah Stacey and Dr. Alex Ferforn for their invaluable help and supports. I would like to thank Dr. Deborah Stacey for her endless times and guidance through out the work of this thesis. Honestly, i don't know what would i have done without you.

I would like to thank my family and parents for supporting me through out this journey. I also would like to thank my girl friend Sandy Zhou for her love, support and understanding during those tough times.

Thank you and thank you all for making this journey possible. 


\section{Contents}

1 Introduction $\quad 1$

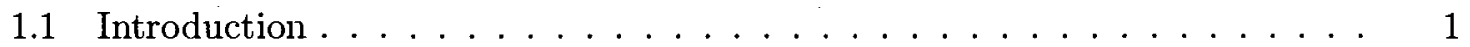

1.2 Thesis Organization $\ldots \ldots \ldots \ldots \ldots$

1.3 Thesis Contributions . . . . . . . . . . . . . . . . 4

2 Literature Review $\quad 5$

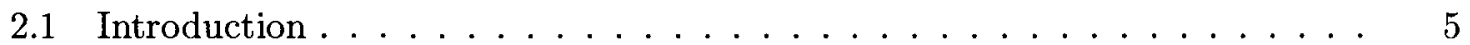

2.2 The Current State Of Research In Face Detection Methods . . . . . . . 5

2.2.1 Knowledge-based Methods . . . . . . . . . . . . 6

2.2.2 Appearance-based Face Detection Methods . . . . . . . . . . . 9

2.3 Texture Features $\ldots \ldots \ldots \ldots \ldots$

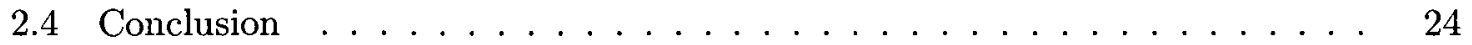

3 Methodology 26

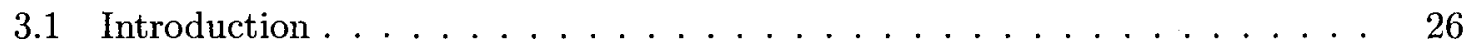

3.1 .1 Performance Measures . . . . . . . . . . . . . . . 26

3.1 .2 Evaluation Protocol . . . . . . . . . . . . . . . . . 30 
3.1.3 Analysis of the face data sets . . . . . . . . . . 31

3.1 .4 System design . . . . . . . . . . . . . . . 34

3.1.5 Experimental design . . . . . . . . . . . . . . 35

3.1.6 Complexity of Our Method . . . . . . . . . . . . . 43

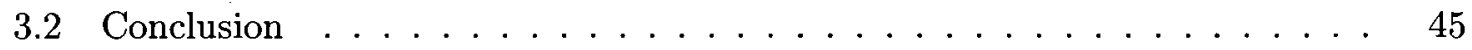

4 Empirical Results and Analysis $\quad 47$

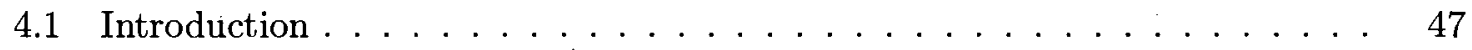

4.2 Experimental results . . . . . . . . . . . . . . . . . 47

4.2.1 The advantages and disadvantages of grey scale features . . . . . 48

4.2.2 The advantages and disadvantages of texture correlation features . : $\quad 55$

4.2 .3 Our inages ..................... 60

4.2.4 Comparison between grey scale and texture features . . . . . . . 64

4.3 Quantization level and its extraction time . . . . . . . . . . . 69

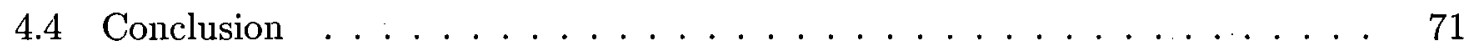

5 Conclusions and Future Work $\quad 72$

5.1 Conclusions . . . . . . . . . . . . . . . . . . . 72

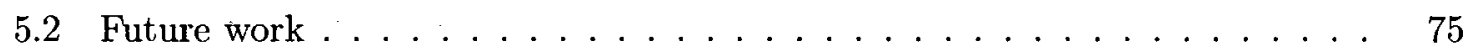

$\begin{array}{ll}\text { A Examples of Face and Non-face images } & \mathbf{8 0}\end{array}$ 


\section{List of Tables}

2.1 Some common inner product kernels for Nonlinear SVMs [16] . . . . . . 17

3.1 Confusion Matrix of an ROC curve . . . . . . . . . . . . . 28

3.2 Confusion Matrix of the ROC curve (figure 3.1) . . . . . . . . . . . . 29

3.3 Confusion Matrix and Performance Metrics of MIT training set [20] . . . . 33

3.4 Confusion Matrix and Performance Metrics of MIT test set [20] . . . . . . 33

4.1 Confusion Matrix of ROC curve (figure 4.2) and performance metrics for our data set 1 at scramble level $22 \ldots \ldots \ldots \ldots$. . . . . . . . . . 50

4.2 Confusion Matrix of ROC curves (figure 4.4) and Performance Metrics for our data set 2 at scramble level $10 \ldots \ldots \ldots \ldots$

4.3 Confusion Matrix of ROC curves (figure 4.5) and Performance Metrics for our data set $3 \ldots \ldots \ldots \ldots \ldots \ldots$

4.4 Confusion Matrix of ROC curve (figure 4.8) and Performance Metrics for our data set 2 at scramble level $10 \ldots \ldots \ldots \ldots \ldots$

4.5 Confusion Matrix of ROC curves (figure 4.9) and Performance Metrics for

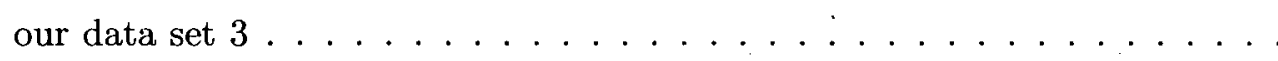




\section{List of Figures}

1.1 Image Samples $\ldots \ldots \ldots \ldots \ldots \ldots \ldots \ldots \ldots \ldots \ldots$

2.1 Quarlet Mosaic Image. Rules are based on the intensity of the facial regions [36] 8

2.2 (a) Horizontal Profile, (b) Vertical Profile $[5] \ldots \ldots \ldots$

2.3 A schematic diagram of a hierachial OCON structure. The global level consists of multiple subnets, and the local level consists of multiple cluster in the subnet $[17] \ldots \ldots \ldots \ldots \ldots \ldots$

2.4 Rowley's System Layout $[13] \ldots \ldots \ldots$. . . . . . . . . . . . 12

2.5 Sung and Poggio distribution-based face model $[30] \ldots \ldots \ldots$

2.6 Overview of Sung and Poggio face detection system.(a) a "canonical face pattern, a 19 x 19 mask for eliminating near boundary pixels of canonical face patterns, and the resulting canonical face pattern after applying the mask., (b) At each scale, the image is divided into many possibly overlapping windows. Each window pattern gets classified as either face or not a face based on a set of local image measurements. (c) The key components of the face pattern recognize" $[30] . \ldots \ldots \ldots \ldots \ldots$

2.7 Optimal hyperplane for linearly separable patterns $\ldots \ldots \ldots$

2.8 In this picture, the circles represent face, the squares represent non-face [10]. 20 
2.9 (a) $4 \times 4$ image with 4 grey tone values 0-3 (b) general form of glcm (c)-(f) calculation of all four $1 \mathrm{glcm}[23] . \ldots \ldots 22$

3.1 An example of an ROC curve . . . . . . . . . . . . . . 29

3.2 Outliners in the face test set of data set $[20] \ldots \ldots \ldots \ldots$

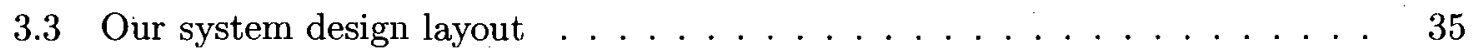

3.4 In subsystem 2, an input image is divided into regions $\mathrm{A}, \mathrm{B}, \mathrm{C}, \mathrm{D}$, and $\mathrm{E}$ for the texture correlation calculation $\ldots \ldots \ldots . \ldots . \ldots 36$

3.5 A face image is transformed into the texture correlation feature space . . . 37

3.6 Two-class classification problems. (a)two-class classification problem of face images and undefined non-face images, (b) two-class classification problem of face images and well-defined non-face images. . . . . . . . . . . . . 38

3.7 Non-face images of MIT CBCL test set $[20] \ldots \ldots$. . . . . . . . . . . 39

3.8 Non-face images of MIT CBCL test set $[20] \ldots \ldots \ldots$. . . . . . . . . 40

3.9 Our first data set . . . . . . . . . . . . . . . . 42

3.10 Our second data set $\ldots \ldots \ldots \ldots \ldots$

4.1 Our experimental results of our system with grey scale features for data set 149

4.2 ROC curves for grey scale features for our data set 1 at scramble level $22 \quad$. 50

4.3 Our experimental results of our system with grey scale features for data set 251

4.4 ROC curve of grey scale features for our data set 2 at scramble level $10 \quad \ldots \quad 52$

4.5 ROC curve for our system with grey scale features for our data set $3 \ldots \ldots 4$

4.6 Our experimental results of our system with texture correlation features for data set $1 \ldots \ldots \ldots \ldots \ldots \ldots \ldots$

4.7 Our experimental results of our system with texture correlation features for

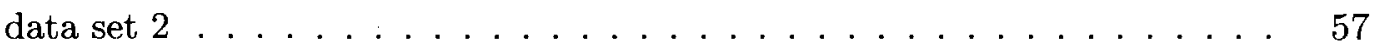


4.8 ROC curve of texture feature correlation features for our data sets 2 at scramble level $10 \ldots \ldots \ldots \ldots \ldots$

4.9 ROC curve for our system with texture correlation features for our data set 359

4.10 Face images are from training set from $[20] \ldots \ldots 1$

4.11 Non-face images of data set 1 are generated from face images that are scram-bled at level $22 \ldots \ldots \ldots \ldots \ldots \ldots$. . . . . . . . . . . . . . . . . .

4.12 Each row of non-face images are generated from face images where pixels are randomly scrambled at levels $25,30,35,40$, and 45 respectively . . . . .

4.13 Non-face images of data set 2 are generated from face images where columns and rows are randomly scrambled at level $10 \ldots \ldots \ldots$

4.14 Each row of non-face images are generated from face images where columns and rows are randomly scrambled at levels $11,12,13,14$, and 15 respectively

4.15 Data set 3 face images generated from face images at scramble level $19 \ldots .64$

4.16 Non-face images from the MIT data set $[20] \ldots \ldots$. . . . . . . . 64

4.17 Non-face images are from MIT data set $[20] \ldots \ldots$. . . . . . . . 65

4.18 Our experimental results of our system with texture correlation and grey scale features for data set $1 \ldots \ldots \ldots 66$

4.19 Our experimental results of our system with texture correlation and grey scale features for data set $2 \ldots \ldots \ldots$. . . . . . . . . . . . 67

4.20 Our experimental results of our system with texture correlation and grey scale features for data set $3 \ldots \ldots \ldots 6$

4.21 ROC curves of various quantization levels $\ldots \ldots \ldots$. . . . . . 70

4.22 Extraction times of correlation texture feature with various quantization levels and grey scale feature for 288 images f . . . . . . . . . . . . . 70

A.1 face images of MIT CBCL training set $[20] \ldots \ldots \ldots \ldots$ 
A.2 face images of MIT CBCL training set $[20] \ldots \ldots \ldots$

A.3 face images of MIT CBCL training set $[20] \ldots \ldots$. . . . . . . . . . . 83

A.4 face images of MIT CBCL training set $[20] \ldots \ldots \ldots$. . . . . . . 84

A.5 face images of MIT CBCL training set $[20] \ldots \ldots \ldots$

A.6 non-face images of our data set1 at scramble level $22 \ldots \ldots$. . . . . . 86

A.7 non-face images of our data set1 at scramble level $28 \ldots \ldots$. . . . . . 87

A.8 non-face images of our data set1 at scramble level $34 \ldots \ldots$. . . . 88

A.9 non-face images of our data set1 at scramble level $40 \ldots \ldots$. . . . . 89

A.10 non-face images of our data set1 at scramble level $46 \ldots$. . . . . . . 90

A.11 non-face images of our data set2 at scramble level $8 \ldots \ldots$. . . . . 91

A.12 non-face images of our data set2 at scramble level $10 \ldots \ldots 2$

A.13 non-face images of our data set2 at scramble level $13 \ldots . \ldots 9$

A.14 non-face images of our data set2 at scramble level $17 \ldots \ldots . \ldots 94$

A.15 non-face images of our data set2 at scramble level $20 \ldots . \ldots 5$ 


\section{Chapter 1}

\section{Introduction}

\subsection{Introduction}

Vision is the most powerful sense that we often take for granted. It allows us to perform our daily tasks such as recognizing people walking on sidewalks without colliding into other people or other objects. Building a system that is capable of mimicking our vision system will allow us to have a wide range of applications. For example, a face recognition system or face detection system can be used in surveillance systems to reduce the operating cost by having the system take over the screening and other automated tasks. A face recognition system can be used to assist blind people in their daily routine tasks. The techniques and understanding gained in this field can be used to improve other pattern classification techniques. The first step in building any face processing system is to build a system that can detect human faces in still images. However, it turns out that it is not a trivial task. Therefore, improving face detection techniques is vital to all face processing applications. It is our motivation and goal of this thesis.

The task of face detection is to find whether or not there are any faces in an image. There are many problems that are associated with face detection. Perhaps, one of the key problems is the fact that the human face is a non-rigid object which has a very high degree 
of variability with respect to different poses, presence or absence of structural components of a face, facial expression, occlusion, image orientation, and other image conditions, etcetera. These unpredictable image conditions can lead to difficulty in encoding and interpreting face patterns; moreover, features extracted from an image are often very complex and have very high dimensionality. Thus, it makes face detection a very challenging task. Considering an image with the size of twenty by twenty pixels, if grey scale features are used, there are $256^{400}$ possible combinations of the grey values in an image. The possible combinations of grey values in an image are approximately ninety eight times more than the world population. This shows a very high dimensionality in representing a small image using the grey scale feature space.

Although much work has been put into the field of face detection, the existing systems still fall far behind human abilities. Many systems proposed only work under specific conditions such as simple backgrounds, adequate lighting conditions, etcetera. The current methods are far too inflexible for any application beyond specific tasks that they are engineered to do. Therefore, improvement is needed to make face detection methods more applicable in real world applications. It is our motivation and the goal of this thesis. There are two primary goals in this thesis. Firstly,we look at the current problems of knowledgebased techniques. Secondly, we examine how texture correlation features can help deal with the problems of grey scale features and examine texture quantization levels on classification performance and their effects on the Support Vector Machine (SVM).

\subsection{Thesis Organization}

Our thesis is organized in five chapters. The first chapter introduces the problems of face detection, the layout of the thesis, and our contributions. The second chapter starts with a literature review of some major face detection methods. We end chapter two by discussing texture features in detail since they are used in our method. In chapter three, we discuss our methodology. We discuss the performance measures and evaluation protocol. We provide 

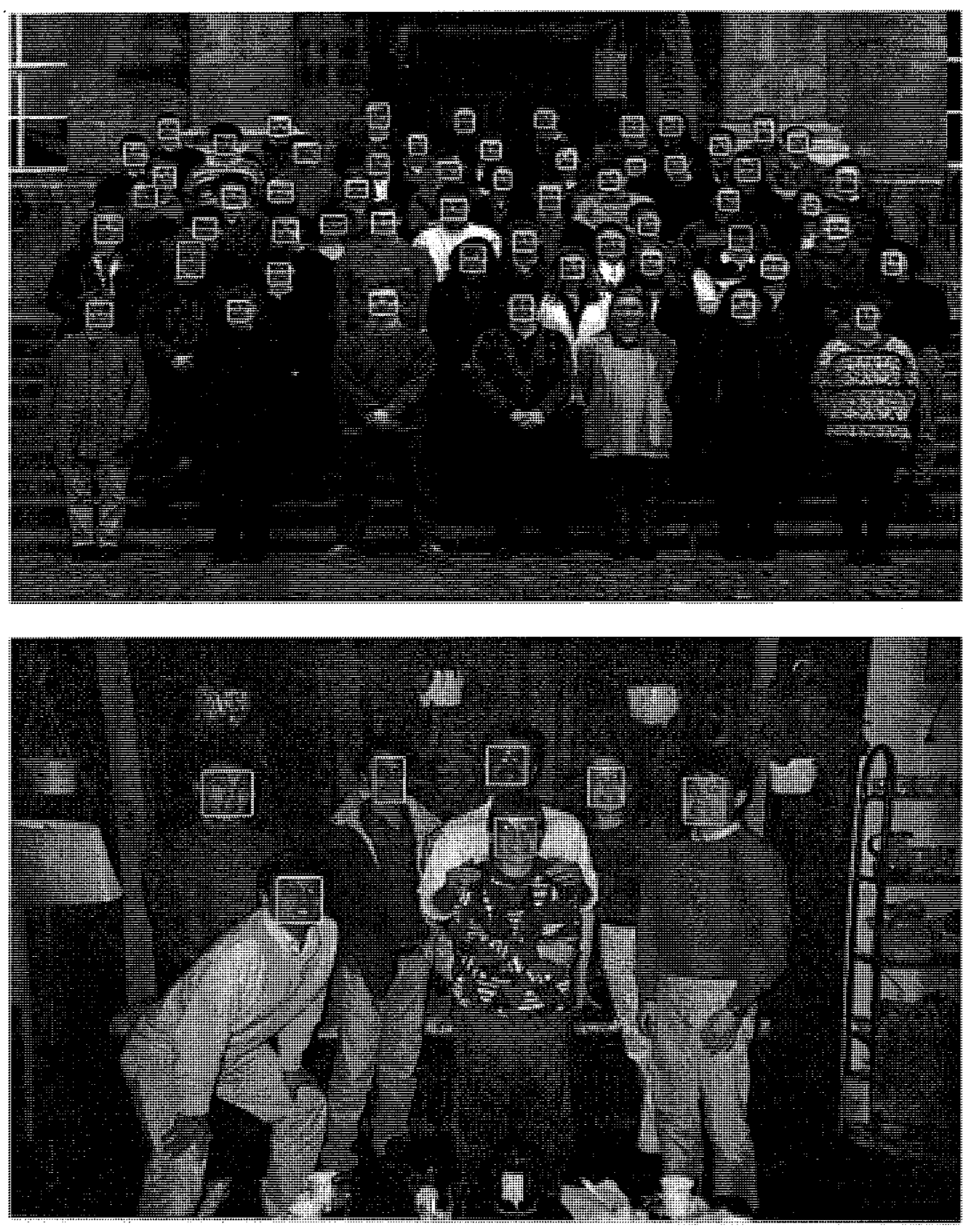

Figure 1.1: Image Samples 
an analysis of the MIT face detection data sets [20]. Finally, we present our method of face detection using the texture correlation feature and Support Vector Machine to solve our problem domain. Chapter four provides our results. Chapter five will give some concluding remarks and discuss possible future work.

\subsection{Thesis Contributions}

In this thesis, our contributions are:

- Firstly, we provide the analysis of the available data sets [20] which has not been done before. We show that there are problems with these data sets.

- Secondly, we construct new data sets which are an improvement on previous data sets [20]. We created non-face images which are closer to face images.

- Thirdly, we suggest the use of evaluation protocols and the receiver operating characteristic (ROC) curve as a means to evaluate the face detection methods. Early methods used different data sets and assumptions and reported only in term of detection rates and false alarm rates which cause a problem in evaluation face detection performance since the system constraints and definitions of detection rates and false alarm rates methods are varied [19].

- Fourthly, we propose a face detection method using texture features [23] and the Support Vector Machines [4]. Our method exploits the use of texture correlation as a function of offsets and the Support Vector Machine[4] as a main classifier. The empirical results show that the texture correlation feature is a lower dimensionality representation compared to the grey scale feature and can have some advantages over grey scale features.

- Lastly, we provide an experiment exploring different quantization levels that can have great effects on classification performance of the texture correlation feature. 


\section{Chapter 2}

\section{Literature Review}

\subsection{Introduction}

The purpose of this chapter is to examine several well known knowledge-based and appearancebased face detection methods. Our motivation is to study current face detection methods. We will discuss some key challenges facing by these methods and lay a groundwork for the work of this thesis. We conclude this chapter by looking at texture features which are used in this thesis.

\subsection{The Current State Of Research In Face Detection Meth- ods}

Face detection is an important step in any face processing application. Researchers in this field have tried to improve and make it possible for commercial applications. A wide range of face processing applications are available in commercial products such as face recognition system which has been employed in digital cameras, surveillance cameras, web camera applications etc. However there are still many problems associated with face detection methods that need to be overcome and make it more robust for real life application. A 
wide range of face detection methods has been discussed in recent survey [19], they can be categorized into four categories as follow:

- Knowledge-based methods. These methods use to construct rules which is used to detect faces. These rules are based on researchers' knowledge of a human face.

- Feature invariant methods. These methods examine face features that exists even when image conditions are changed such as lighting, and pose etc.

- Template matching methods. Several face templates or patterns are created and used as a mean to detecting faces. The similarity between the face patterns and input image are computed for detection.

- Appearance-based methods. These methods are constructed by training images which capture facial variations. The trained models are used for face detection.

These techniques use a wide range of technologies and theories. For these reasons, we would like to focus our research attention on two face detection methods which are knowledge-based and appearance-based methods instead of looking at a wide range of face detection methods. Our goals is to highlight some key challenges faced by these methods and lay a foundation on shaping the work of this thesis. A detailed discussion of these methods is presented in the next subsections.

\subsubsection{Knowledge-based Methods}

Knowledge-based face detection methods are based on researchers' knowledge of a human face. Simple rules are constructed to describe a typical human face. Knowledge-based technique uses these rules to detect a human face. An image which has agreed with these rules is considered to be a human face.

Yang and Huang proposed a hierarchical knowledge-based approach for face detection [36]. Their system consists of three levels of rules. The rules at higher level describe a 
general description of a human face - for example what a face looks like whereas the rules at the lower level describe more details by using facial features. At level one and two, rules are based on mosaic image whereas at level three, rules are based on the use of edge detection methods on the mouth and nose regions. Mosaic image (multiresolution image) is obtained by decreasing the resolution of the original image. A mosaic image consists of cells. The value of each cell is an average grey scale value of pixels in that cell. At level one, the input image is scanned globally to find all possible locations and sizes for human face candidate. Level one rules are based on mosaic image called the quartet. Quartet mosaic image is defined as having characteristic of figure 2.1 below. An example of these rules are based on figure $2.1[36]$ :

- The center part of the face (the quartet) has four cells with a uniform grey level

- The upper round part of a face (the lightly shaded part; there are eight cells altogether) has a uniform grey level

- There is a large difference in average grey levels of the center part and the upper round part.

If the face candidate is passed all the rules at level one, it is fed into level two for screening. Similarly, level two rules are based on octet mosaic image. These rules examine facial regions in more details such as nose, eye, and mouth region. At each region, rules are created based on the intensity characteristic of the regions. The rules based on the quartet and octet are obtained by comparing and observing the intensity of the mosaic cells. As Face candidate successfully passed through the screening stage in level one and two, it is passed into level three. At level three, histogram equalization, multi-binarization, and bidirectional edge tracking [36] are used. The edge features of eyes and mouth region are extracted and used to create rules at this level. The main purpose of these rules are used to reject the false negative face candidate from level two.

Kotropoulous and Pitas proposed another knowledge-based method [5] which aims to improve Yang and Huang method [36]. Yang and Huang method [36] is said to be 


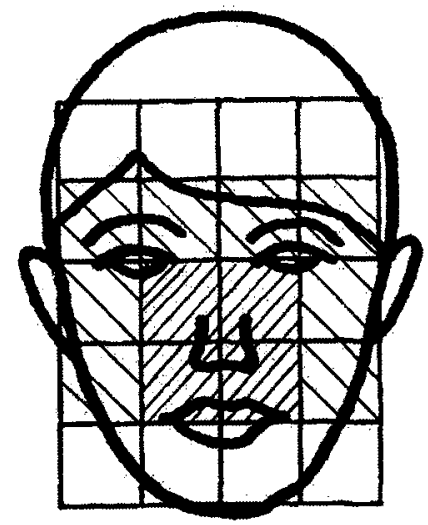

Figure 2.1: Quarlet Mosaic Image. Rules are based on the intensity of the facial regions [36] computational intensive in determining the cell dimensions for quartet image by iteratively looking at the entire possible cells. Instead of using the mosaic qualtet and octet. They use the horizontal and vertical image profile to estimate the cell dimensions in quartet image. The horizontal image profile is obtained by averaging all pixels intensities in each image column. The result of horizontal image profile is a vector with the number of elements corresponding to number of columns or horizontal pixels of the image. Two significant local minima in the vector are observed corresponding to the left and right side of the head (figure 2.2(a)). The distance between them is about $4 \mathrm{~m}$ where $\mathrm{m}$ denotes as horizontal quartet cell dimensions. Similarly, the vertical image profile can be obtained by averaging all pixels intensities in each image row. The result is a vector with number of elements corresponding to the number of rows or vertical pixels of the image. The significant local minima in the vertical profile correspond to hair, eyebrows, eyes, mouth and chin can be located by observation. The quartet cell dimension in vertical direction can be determined by measuring the distance between eyes and upper lip of the vertical profile of the image (figure 2.2(b)). Unlike method [36], detection rules are based on these local minima. The method has been tested with a set of faces in frontal view extracted from image database [21]. The detection rate is 86.5 percent of successfully identifying facials features specifically 
eyebrows, eyes, nostrils, noses and mouths.

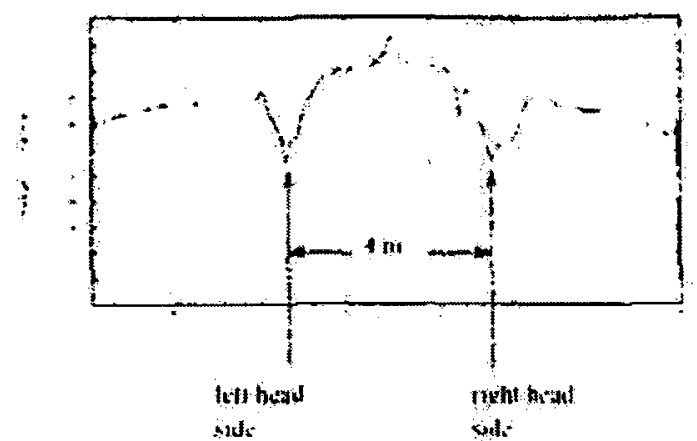

(a)

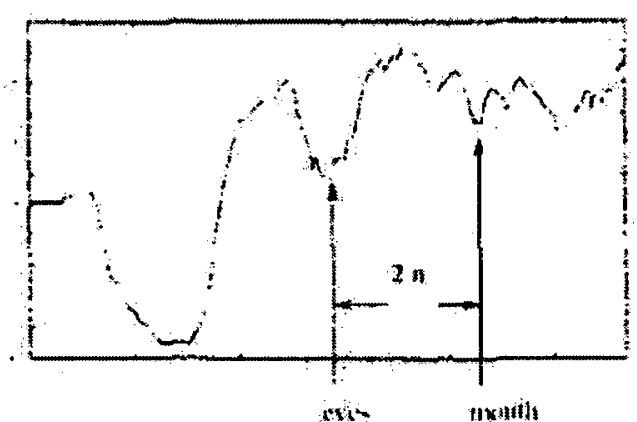

(b)

Figure 2.2: (a) Horizontal Profile, (b) Vertical Profile [5]

\subsubsection{Appearance-based Face Detection Methods}

\subsubsection{Neural Network Methods}

Neural network have been applied successfully in speech recognition, pattern recognition, and other adaptive control techniques. Face detection can be considered a special case of pattern recognition problem where only two classes are considered. While neural network can captured the complex face patterns, it needs to be fine tune and have good training data to get good results.

Lin et al. proposed a face detection method using Probabilistic Decision-based Neural Network (PDBNN) [25] which adopts the structural properties of Decision Based Neural Network (DBNN) [17]. The system is designed to perform face detection and facial recognition. To simplify the scope of this survey, we shall examine the face detection subsystem. The PDBNN face detection system is divided into two stages. The first stage involves in taking a sample from an image. The sampling process involves in scaling down the original 
$320 \times 240$ pixel image by a factor of 0.7 and scanning a window with a size of $12 \times 12$ pixel across the image by 1 pixel at a time. The window sample is processed with histogram equalization to remove the unwanted lighting conditions of the image. The features extracted from the window contains the intensity and edge information of facial region that contains eyebrows, eyes, and nose. The second stage is to feed the feature vectors into PDBNN to determine if it is a face or not and reports the location of the face accordingly. A confident score is used by PDBNN. If the score is above some predetermined threshold, then face is detected. According to [19], "The architecture of PDBNN is very similar to a Radial Basis Function neural network (RBF) with modified learning rules and probabilistic interpretation. It adopts a One-class-in-One-Network (OCON), where one subnet is designated for one class". The empirical results on a set of 23 images provided by Sung and Poggio [30] showed that PDBNN has the inferior performance compared to other leading neural network-based face methods [30]. It is argued that PDBNN used a smaller training set than [30] and [13]

Perhaps, the most well-known work for face detection is done by Rowley et al. [13] [14] [12]. A multilayer neural network is used to learn the face and non-face patterns. Rowley et al's system composes of 3 components: preprocessing module, neural network module and a decision making module. The first component involves with pyramid sampling model and preprocessing image. A small window with the size of $20 \times 20$ pixels is used to sample or scan across the image pixel by pixel. In order to detect the face larger than the window size, the image is scaled down by factor of 1.2. The window is applied to the image at all scales. Before the window is fed into the next component, a linear function will approximate the overall brightness of the window and can be subtracted from the window to compensate for a variety of lighting conditions. The histogram equalization is then performed to expand the range of intensities in the window. It improves the contrasts of the windows and also compensates for differences in camera input. The result of the window is fed into neural network (second component) for face detection. The results are close to -1 for non-face image or close to 1 for face image. The system uses nearly 1,050 face samples from Carnegie 


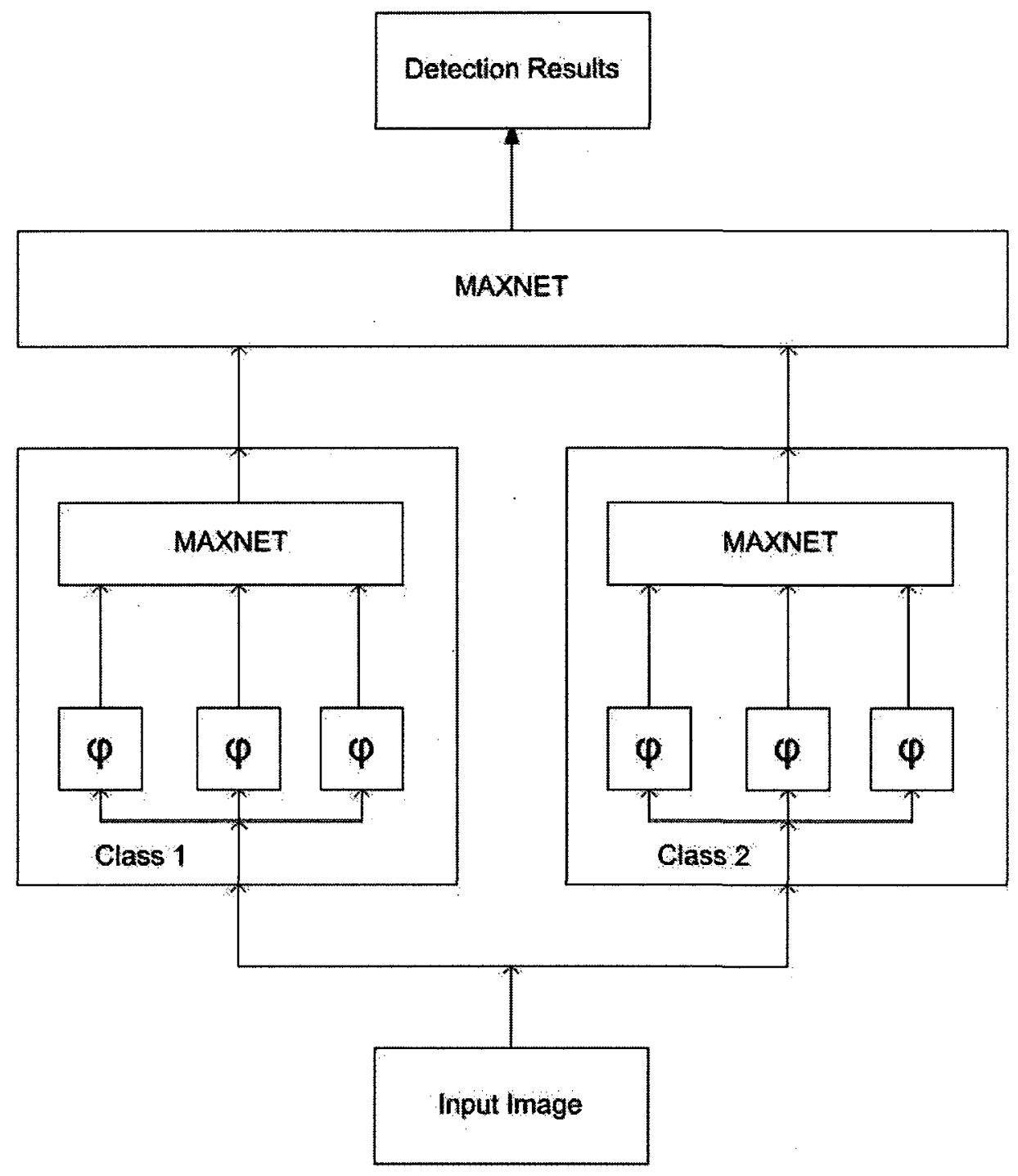

Figure 2.3: A schematic diagram of a hierachial OCON structure. The global level consists of multiple subnets, and the local level consists of multiple cluster in the subnet [17]

Mellon University (CMU) and Harvard image database to train the network. In each training set, 15 face samples are generated from an original image by rotating the image up to 10 
degrees, scaling between 90 percent and 110 percent, translating half a pixel and mirroring. Each sample is then preprocessed with lighting correction and histogram equalization.

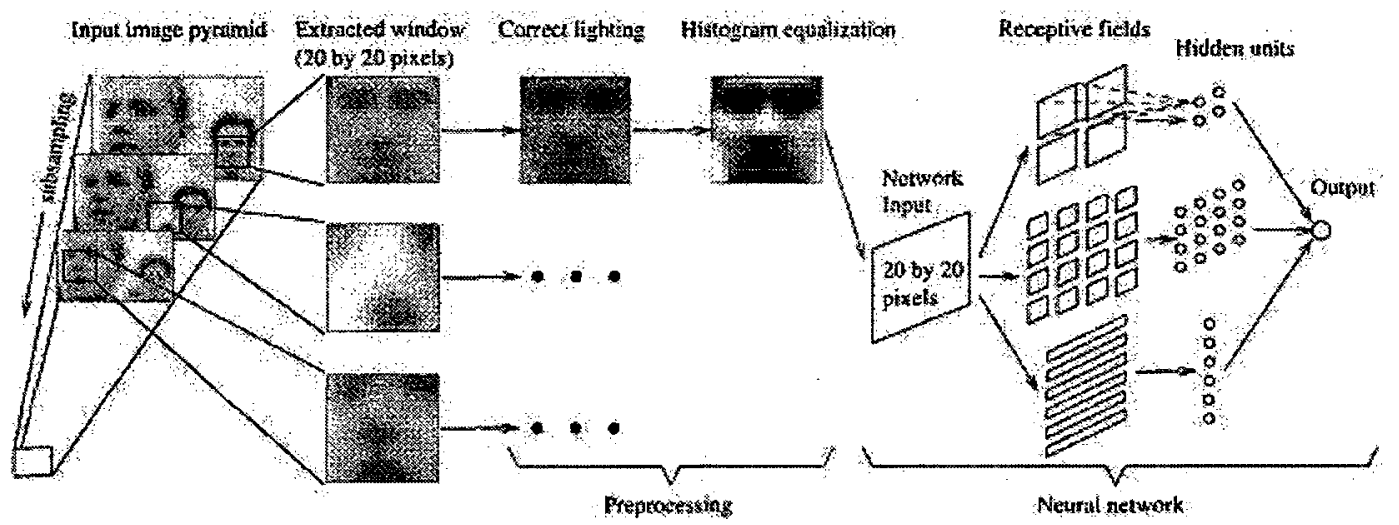

Figure 2.4: Rowley's System Layout [13]

The training method is adopted from Sung and Poggio [30] to solve the imbalances between face and non-face data sets. The output is the combination of the outputs of multiple networks. Rowley et al. [13] systems reported to have less computational cost than Sung and Poggio's system. They have higher detection rates based on a test set of 24 images containing 144 faces. One limitation of Rowley et al' method [13] is only able to detect upright, frontal faces. To improve this limitation, Rowley et al. [12] adds a router network which examines other facial orientations. The new system has a lower detection rate than the previous system for upright face images. The result of 76.9 percent of faces successfully classified over two large test sets are reported with a small number of false positives.

Sung and Poggio developed a distribution-based system for face detection [30], [29]. The system consists of 3 components. These are the preprocessing component, distributionbased model and a multilayer perceptron classifier. The first component involves in normalization steps such as image resizing, masking, illumination gradient correction, and histogram equalization. Image resizing involves in reducing the image size to $19 \mathrm{x} 19$ pixels 
while preserving distinctive visual features. In order to reduce unwanted background artifacts, masking is used. Illumination gradient correction is used to reduce heavy shadows caused by extreme lighting conditions, and histogram equalization is the applied to compensate for changes in illumination brightness and differences in camera. After the face and non-face examples are preprocessed, the distribution-based model is obtained by using a modified k-mean clustering algorithm. The model is partitioned the training images into six face and non-face clusters.

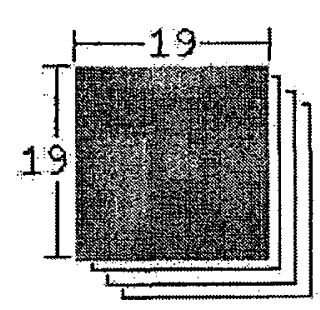

Frontal Face Pattem samples to epproximate vector subspace of conorical tace views

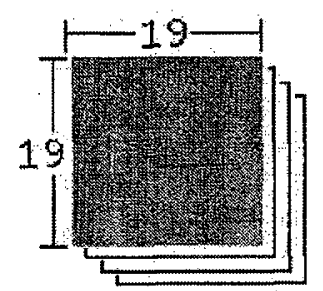

Special Non-Fece Pattem seimples ta refine vector subspace boundaries of canonical face views
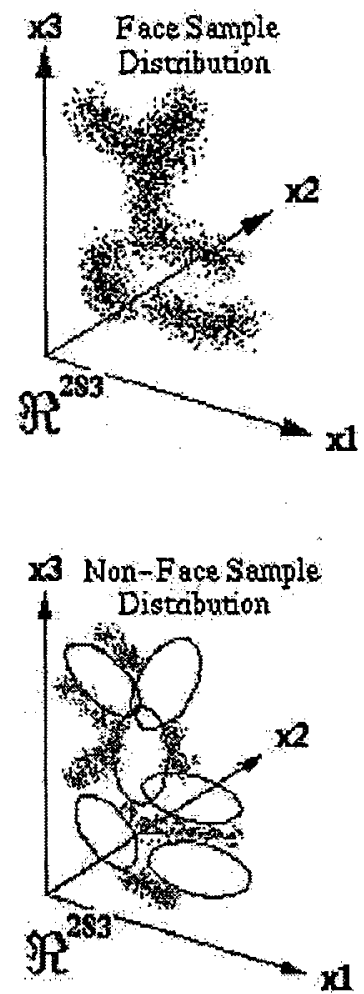
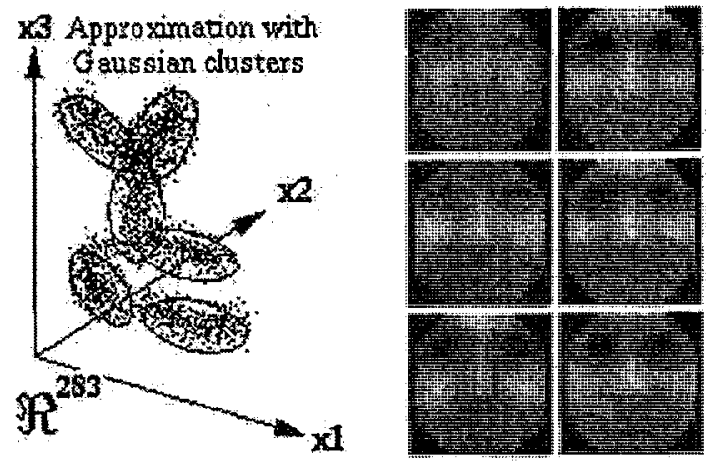

Face Centroids

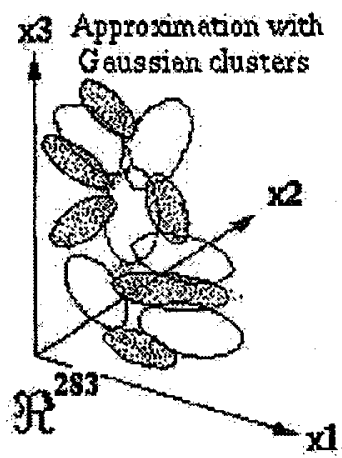

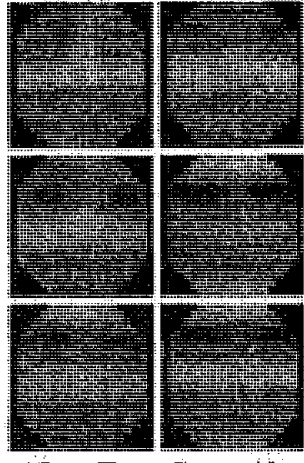

Non-Face Centroids

Figure 2.5: Sung and Poggio distribution-based face model [30]

Next, according to [30], "Each cluster is represented as a multidimensional Gaussian function with a mean or centroid image and a covariance. Two distance metrics are computed between an input image pattern and the prototype clusters. The first distance component is the normalized Mahalanobis distance between the test pattern and the clus- 
ter centroids, measured within a lower-dimensional subspace spanned by the cluster's 75 largest eigenvectors. The second distance component is the Euclidean distance between the test pattern and its projection onto the 75-dimensional subspace. The second distance component is used to accounts for pattern differences not captured by the first distance component" [30]. The final step is to use Multi Layer Perceptron classifier to classify face patterns and non-face patterns based on twelve pairs of distances. The classifier is trained using standard back propagation from a data set with 47,316 window patterns.

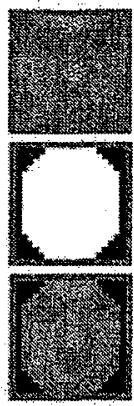

(a)

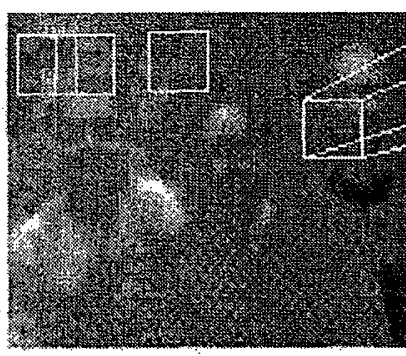

Input Image

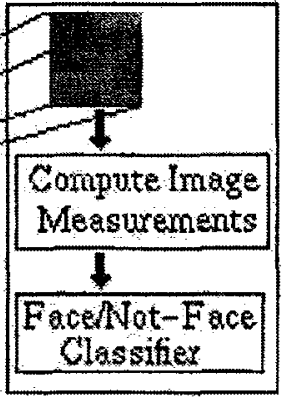

Eace Pattem Recognizer

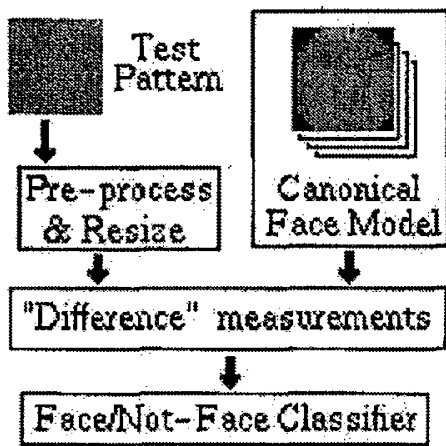

(c)

Figure 2.6: Overview of Sung and Poggio face detection system.(a) a "canonical face pattern, a $19 \times 19$ mask for eliminating near boundary pixels of canonical face patterns, and the resulting canonical face pattern after applying the mask., (b) At each scale, the image is divided into many possibly overlapping windows. Each window pattern gets classified as either face or not a face based on a set of local image measurements. (c) The key components of the face pattern recognize" [30].

There are 4,150 positive examples of face images and 43,166 non-face images. Note that there is an imbalance in face and non-face images. Sung and Poggio solves this problem by using a bootstrap technique that adds images into the training set. First, the training process starts with a small set of non-face examples in the training set. Second, the MLP classifier is trained with the current data set. Third, the classifier is tested on some random 
images. It collects all the non-face patterns that the system wrongly classifies as faces. These non-face patterns are added into the training data set as new non-face images. Fourth, return to second step. The bootstrap technique ends when balance data set is obtained. At the end of each iteration, the set of "non-face" patterns are enlarged with the new false positive ones. Bootstrap technique can also use to enlarge face data set.

\subsubsection{Support Vector Machine Methods}

Support Vector Machines (SVMs) Support vector machines are invented and derived from statistical learning theory by Vapnik [35]. The main idea of the SVMs is to find a hyper plane as a decision surface in such a way that a margin of separation between two classes is maximized. SVMs' principle is based on structural risk minimization [35]. This principle is based on the fact that generalization error rate is bounded by the sum of training error rate and a term that depends on the Vapnik-Chervonenkis dimension [35]. In this section, we look at two cases: linear separable patterns and nonlinear separable patterns.

Linear Separable Patterns The most important aspect of SVMs is to construct a hyperplance to separate linear separable patterns. Perhaps, the best way is to construct a hyperplane in such a way that a margin of separation between two classes is maximized. It is shown in [35]that we can find the optimal hyperplane by maximizing the objective function using method of Larange multiplier which shown as follow: "Given the training example $\left(x_{i}, d_{i}\right)_{i=1}^{N}$ find the Lagrange multiplier $\left\{\alpha_{i}\right\}_{i=1}^{N}$ that maximize the objective function:

$$
Q(\alpha)=\sum_{i=1}^{N} \alpha_{i}-\frac{1}{2} \sum_{i=1}^{N} \sum_{j=1}^{N} \alpha_{i} \alpha_{j} d_{i} d_{j} x_{i}^{T} x_{j}
$$

subject to the constrains
1. $\sum_{i=1}^{N} \alpha_{i} d_{i}=0$
2. $0 \leq \alpha_{i} \leq C$ for $i=1,2, \ldots, N$ 


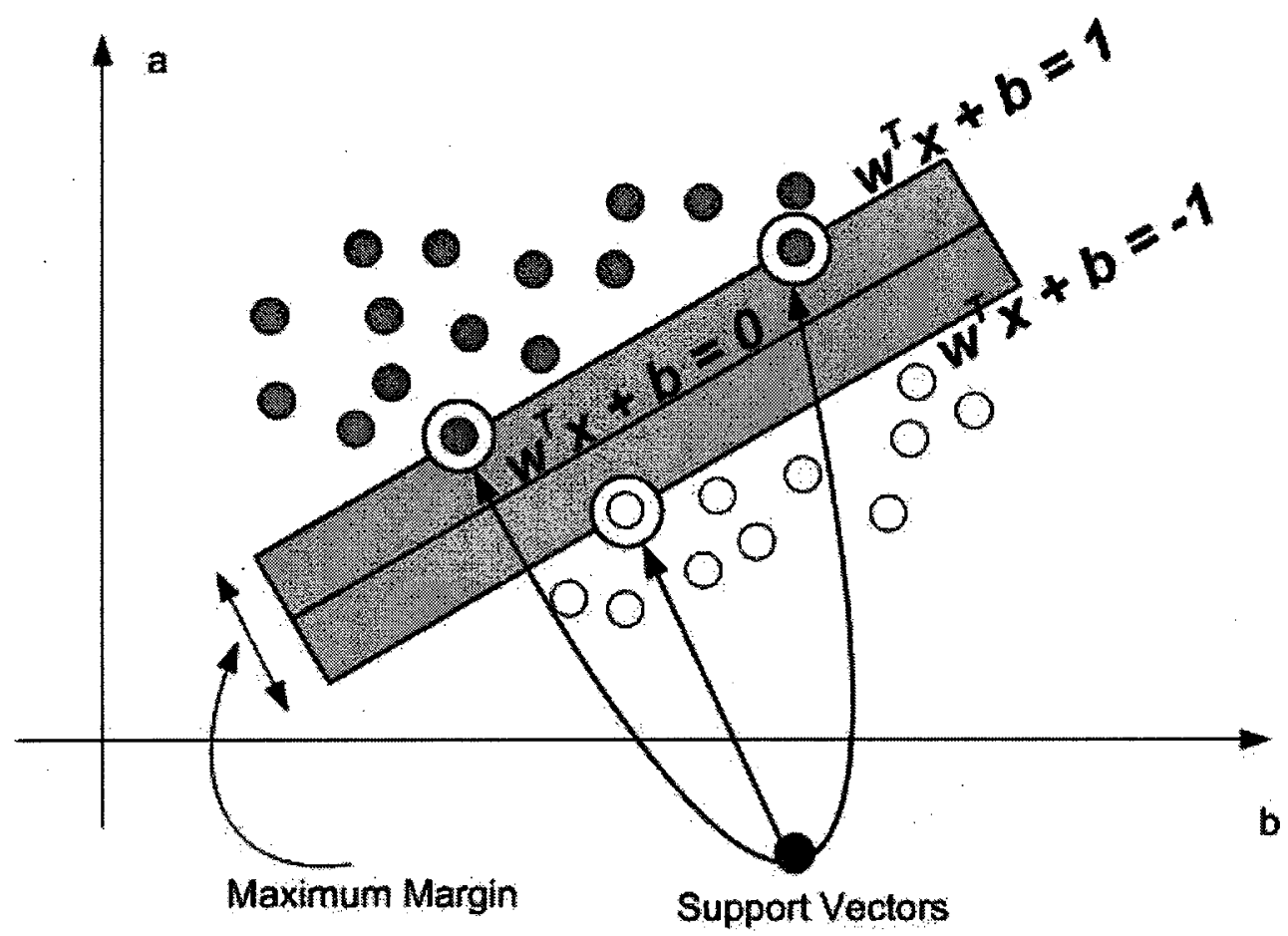

Figure 2.7: Optimal hyperplane for linearly separable patterns

where $C$ is a user-specified positive parameter" [16]

In other words, finding an optimal hyperplane is to solve quadratic objective function which is a quadratic programming problem with linear and bound constrains. The above equation shows that maximizing the objective function depends only on the inner products of the input data.

Non-Linear Separable Patterns To solve the non-linear patterns, we can construct SVMs based on two operations:

1. Transforming the input pattern into higher dimensional hidden space so that they are linearly separable.

2. Finding the optimal hyperplane for separating the features space in step one 
For the first step, the transformation is done by the inner product kernel. The kernel has to satisfy the Mercer's theorem. The second step is similar to the linearly separable case. The objective function can be rewritten with the inner product replaced by the inner kernel product as follow:

"Given the training example $\left(x_{i}, d_{i}\right)_{i=1}^{N}$ find the Lagrange multiplier $\left\{\alpha_{i}\right\}_{i=1}^{N}$ that maximize the objective function:

$$
Q(\alpha)=\sum_{i=1}^{N} \alpha_{i}-\frac{1}{2} \sum_{i=1}^{N} \sum_{j=1}^{N} \alpha_{i} \alpha_{j} d_{i} d_{j} K\left(x_{i}, x_{j}\right)
$$

subject to the constrains
1. $\sum_{i=1}^{N} \alpha_{i} d_{i}=0$
2. $0 \leq \alpha_{i} \leq C$ for $i=1,2, \ldots, N$

where $C$ is a user-specified positive parameter" [16]

The table below shows some common inner product kennels for nonlinear SVMs.

\begin{tabular}{|l|l|l|}
\hline Type of SVMs & $\begin{array}{l}\text { Kernel } K\left(x, x_{i}\right), i= \\
1,2,3, \ldots, N\end{array}$ & $\begin{array}{l}\text { Comments } \\
\text { priori by the user }\end{array}$ \\
\hline Polynomial learning machine & $\left(x^{T} x_{i}+p\right)^{p}$ & $\begin{array}{l}\text { The width } \sigma, \text { common } \\
\text { to all the kernels is spec- } \\
\text { ified a priori by user }\end{array}$ \\
\hline Radial basis function network & $\exp \left(-\frac{1}{2 \sigma^{2}}\left\|x-x_{i}\right\|^{2}\right)$ & $\begin{array}{l}\text { Mercer's theorem is sat- } \\
\text { isfied only for some } \\
\text { value } \beta_{0} \text { and } \beta_{1}\end{array}$ \\
\hline Two-layer perceptron & $\tanh \left(\beta_{0} x^{T} x_{i}+\beta_{1}\right)$ & \\
& &
\end{tabular}

Table 2.1: Some common inner product kernels for Nonlinear SVMs [16] 
SVMs Algorithm A general form of SVMs algorithm is shown below:

1. Choose a kernel.

- If the data is linear separable pattern, go to step 2 .

- Else choose a inner product kennel function for nonlinear case.

2. Choose a parameter C.

3. Solve the objective function (2.1) for linear case and (2.2) for nonlinear case for Lagrange multiplier $\alpha$ ( Can be done by genetic algorithm or other software packages)

4. Construct the optimal hyperplane.

For linear case, hyperplane has a form:

$$
g(x)=w^{T} x+b
$$

where optimal $w=\sum_{i=1}^{N} \alpha_{i} d_{i} x_{i}$ and $b=\frac{1}{d_{i}}-w^{T} x$

For nonlinear case, hyperplane has a form:

$$
g(x)=w^{T} \varphi(x)+b
$$

where optimal $w=\sum_{i=1}^{N} \alpha_{i} d_{i} \varphi\left(x_{i}\right), b=\frac{1}{d_{i}}-w^{T} \varphi(x)$ and $\varphi(x)$ is a mapping of $\mathrm{x}$ in feature space

5. For every input $x, g(x) \geq 1$ for $d=+1, g(x) \leq-1$ for $d=-1$

There are some issues associated with SVMs. SVMs rely on the inner product or inner product kernel to maximize the objective function However, as the training set increases to very large numbers, to solve the objective function for Lagrange multiplier becomes difficult because we need a large memory to calculate the large number of inner products or inner product kernel. For nonlinear pattern cases, choosing right the kernel and kernel's parameters is extremely important. Because the kernel is responsible for transforming the 
input data into the feature space that is linearly separable. If SVMs' kernel is ineffective, a better kernel is needed for proper transformation.

Support Vector Machine Methods Osuna et al method [10] was the first SVM method for face detection. This method addresses a problem of SVM with large training set. The computation for SVMs with large data set is both time and memory consuming. To overcome this situation, Osuna et. al [10] propose a method of decomposing the large problem into a smaller problems. A large scale Quadradic Programming (QP) problem can be solved. The algorithm is proved to be converged at global optimum. Osuna et al system structure is very similar to Sung and Poggio's. They use Sung and Poggio preprocessing stage. To classify the pattern, the SVMs are used instead. The location of a face is based on the output of the SVM.

Osunaet et al. proposed and developed an efficient method to train an SVM for large data set. Their system reported to have lower error rates and run about 30 times faster than Sung and Poggio' system [30] by using two test sets of 10,000,000 test patterns of 19 x 19 pixels.

\subsection{Texture Features}

Many image features have been used in face detection methods. However texture feature has not been paid much attention to. It has only been used in [1] [7]. For this reason, our motivation is to examine texture feature and its application for face detection method. Unlike other features, texture feature has its uniqueness in a sense that even though it's easy for us to recognize texture, it is difficult to define texture pattern. Here are some definitions of texture used in the literature:

- "The notion of texture appears to depend upon three ingredients: (i) some local 'order' is repeated over a region which is large in comparison to the order's size, (ii) the order consists in the non-random arrangement of elementary parts, and (iii) the parts 


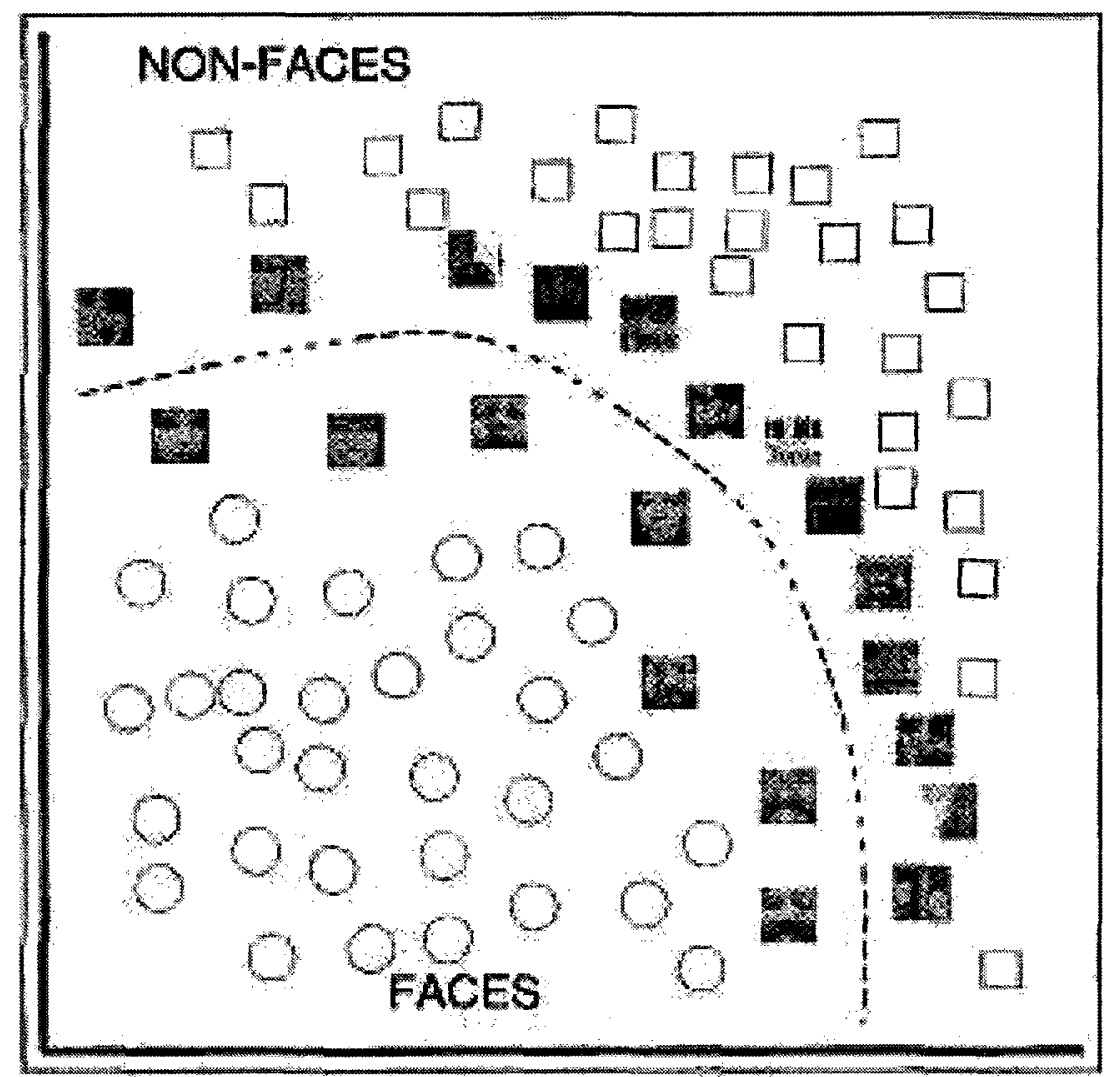

Figure 2.8: In this picture, the circles represent face, the squares represent non-face [10].

are roughly uniform entities having approximately the same directions everywhere within the textured region." [15]

- "A region of an image has a constant texture if a set of local statistics or other local properties of the picture function are constant, slowly varying, or approximately periodic." [26]

- "We may regard texture as what constitutes a macroscopic region. Its structure is simply attributed to the repetitive patterns in which elements or primitives are arranged according to a placement rule." [32] 
- "Texture is an apparently paradoxical notion. On the one hand, it is commonly used in the early processing of visual information, especially for practical classification purposes. On the other hand, no one has succeeded in producing a commonly accepted definition of texture. The resolution of this paradox, we feel, will depend on a richer, more developed model for early visual information process- ing, a central aspect of which will be representational systems at many different levels of abstraction. These levels will most probably include actual intensities at the bottom and will progress through edge and orientation descriptors to surface, and perhaps volumetric descriptors. Given these multi-level structures, it seems clear that they should be included in the definition of, and in the computation of, texture descriptors. " [37]

- "Texture is defined for our purposes as an attribute of a field having no components that appear enumerable. The phase relations between the components are thus not apparent. Nor should the field contain an obvious gradient. The intent of this definition is to direct attention of the observer to the global properties of the display i.e., its overall coarseness, bumpiness, or fineness. Physically, nonenumerable (aperiodic) patterns are generated by stochastic as opposed to de- terministic processes. Perceptually, however, the set of all patterns without obvious enumerable components will include many deterministic (and even periodic) textures." [22]

A lengthy list of texture definitions was presented by Coggins [3]. It shows the difficulty to give a general definition of texture. Many texture features have been studied in the literature for examples statistical texture methods [23], geometrical methods [33], model based methods [9], [6] and signal processing methods [2], [18], [8]. In this thesis, we will only focus on statistical method, specifically, the textural features for image classification proposed by Robert H. Haralick [23]. This is a second-order statistic method. It takes the spatial domain image and statistical nature of texture into account. Haralick proposed the use of gray level co-occurrence matrices (GLCM). Four matrices are calculated to describe different orientation of the textures. These matrices are used to describe the relationship of the pixels that are adjacent to another pixel horizontally, vertically, diagonally in both 
directions. Let consider the following grey image figure 2.9(a) used by Harlick. GLCM texture features normally consider the relation between the two pixels at a time. One is called reference pixel, and another called neighbor pixel. In this example, we look at the horizontal direction (from left to right and right to left). Let consider the figure 2.9, we need to calculate the frequency of occurrence of $(0,0),(0,1)$, and so forth and record the number of occurrences in the general GLCM. (Please note that $(0,0)$ reads as a reference pixel of ' 0 ' value and a neighbor pixel with value ' 0 ') Therefore, the horizontal GLCM has the form of figure 2.9(c) Similarly, the GLCMs for other directions can be calculated in

\begin{tabular}{|l|l|l|l|}
\hline 0 & 0 & 1 & 1 \\
\hline 0 & 0 & 1 & 1 \\
\hline 0 & 2 & 2 & 2 \\
\hline 2 & 2 & 3 & 3 \\
\hline
\end{tabular}

(a)

$$
P_{H}=\left(\begin{array}{llll}
4 & 2 & 1 & 0 \\
2 & 4 & 0 & 0 \\
1 & 0 & 6 & 1 \\
0 & 0 & 1 & 2
\end{array}\right)
$$

(c)

$$
{ }^{135^{\circ}} P_{L O}=\left(\begin{array}{llll}
2 & 1 & 3 & 0 \\
1 & 2 & 1 & 0 \\
3 & 1 & 0 & 2 \\
0 & 0 & 2 & 0
\end{array}\right)
$$

(e)

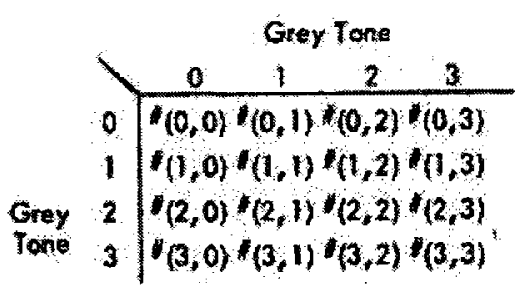

(b)

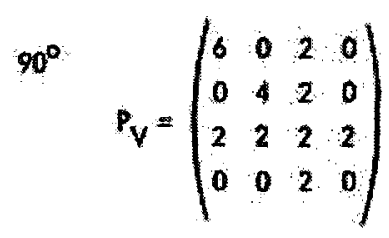

(d)

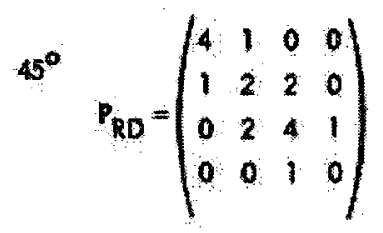

(f)

Figure 2.9: (a) $4 \times 4$ image with 4 grey tone values 0-3 (b) general form of glcm (c)-(f) calculation of all four $1 \mathrm{glcm}$ [23].

a similar manner. Once, the GLCMs are calculated. Fourteen features that based on the 
GLCMs can be calculated as follow:

1. Angular Second Moment:

$$
f_{1}=\sum_{i} \sum_{j}\{p(i, j)\}^{2}
$$

2. Contrast:

$$
f_{2}=\sum_{n=0}^{N g-1} n^{2}\left\{\sum_{i=1}^{N g} \sum_{i=1}^{N g} p(i, j)\right\}
$$

3. Correlation:

$$
f_{3}=\frac{\sum_{i} \sum_{j}(i j) p(i, j)-\mu_{x} \mu_{y}}{\sigma_{x} \sigma_{y}}
$$

4. Sum of Squares: Variance

$$
f_{4}=\sum_{i} \sum_{j}(i-\mu)^{2} p(i, j)
$$

5. Inverse Difference Moment:

$$
f_{5}=\sum_{i} \sum_{j} \frac{1}{1+(i-j)^{2}} p(i, j)
$$

6. Sum Average:

$$
f_{6}=\sum_{i=2}^{2} N g i p_{x+y}(i)
$$

7. Sum Variance:

$$
f_{7}=\sum_{i=2}^{2} N g\left(i-f_{s}\right)^{2} p_{x+y}(i)
$$

8. Sum Entropy:

$$
f_{8}=-\sum_{i=2}^{2 N g} p_{x+y}(i) \log \left\{p_{x+y}(i)\right\}
$$

9. Entropy:

$$
f_{9}=-\sum_{i} \sum_{j} p(i, j) \log \left\{p_{x+y}(i)\right\}
$$

10. Difference Variance:

$$
f_{10}=\text { varianceof } p_{x-y}
$$


11. Difference Entropy:

$$
f_{11}=-\sum_{i=0}^{N g-1} p_{x-y}(i) \log \left\{p_{x-y}(i)\right\}
$$

12. Information measures of correlation 1 :

$$
f_{12}=\frac{H X Y-H X Y 1}{\max \{H X, H Y\}}
$$

13. Information measure of correlation 2 :

$$
f_{13}=(1-\exp [-2.0(H X Y 2-H X Y)])^{\frac{1}{2}}
$$

14. Maximal Correlation Coefficient:

$$
f_{14}=(\text { Second largest eigenvalue of } \mathrm{Q})^{\frac{1}{2}}
$$

where HX and HY are entropies of $p_{x}$ and $p_{y}$ and

$$
\begin{aligned}
& H X Y=-\sum_{i} \sum_{j} p(i, j) \log (p(i, j)) \\
& H X Y 1=-\sum_{i} \sum_{j} p(i, j) \log \left\{p_{x}(i) p_{y}(j)\right\} \\
& H X Y 2=-\sum_{i} \sum_{j} p_{x}(i) p_{y}(j) \log \left\{p_{x}(i) p_{y}(j)\right\} \\
& Q(i, j)=\sum \frac{p(i, k) p(j, k)}{p_{x}(i) p_{y}(k)}
\end{aligned}
$$

\subsection{Conclusion}

This review allows us to examine current knowledge-based face detection methods and appearance-based face detection methods. For hierarchical knowledge-based methods [36], a high detection rate of 83 percent correctly located human face on a test set of 60 pictures is reported. The problems that associated with this method are the fact that the rules are created based on a training set of 40 images. If there is a large variation in the image intensity of the images in the example set, it will affect the rules at each level. Thus, it will affect the overall performance of this technique. Another knowledge based method [5] is to improve hierarchical knowledge-based method [36] by using image profile feature. Though image profile feature is fast to extract, it can be susceptible to low resolution image and 
other image conditions that can cause local minima difficult to identify. The use of image profile might not work for the case where pixels' intensities are much varied in the sample data set thus the variance of pixels' intensities must be taken into account. In general, knowledge-based method is simple to construct. However, it is difficult to construct rules that fit all situations especially where we have large data sets that have large variations of image intensity. In the worst scenarios, one rule can negate other rules which will affect the overall classification performance. For appearance-based methods, we learn that most appearance-based methods have 2 to 3 stages: Sampling and preprocessing stage, Neural network or SVMs stage, and face location stage. Even though appearance-based methods are well understood, there are many problems associated with them such as:

- Evaluation and constraint problems

- Data sets problems.

The problems of evaluation is the fact that appearance-based methods use different data sets and different constraints. They are very difficult to evaluate. Plus, most of data sets are not available. The existing evaluation techniques are different from one method to another one. It needs to be carefully considered and constructed. Perhaps, a standard evaluation techniques should be created to fairly evaluate the system performance. More over, many appearance-based methods assume that they have good data sets that adequately capture the variability of the face images. Therefore the analysis of these data sets have often ignored by the researchers. Whether we have a good or bad data set is very important because data sets play a crucial role in modeling appearance-based method because it defines problem domain thus the need of analyzing and paying attention on these data sets is paramount to the improvement of the appearance-based methods. Not only does it allow us to have a better understanding of our problem domain but also allows us to evaluate or test our system better. 


\section{Chapter 3}

\section{Methodology}

\subsection{Introduction}

In this chapter, we construct our methodology for face detection. Our methodology is based on previous work and provides some answers to the problems that we found in the literature review. There are three primary goals in this chapter. The first goal is to define the performance measure and to construct an evaluation protocol that we will use in our method. The second goal is to address the problems of the current data sets and to construct new data sets. We present our problem domain by providing the definition of face and nonface images. These definitions are important because they pose a specific problem domain that we want to deal with as opposed to a general problem of face detection. The third goal is to examine the properties of texture correlation features and their effectiveness for utilizing the Support Vector Machine for face detection for our data sets.

\subsubsection{Performance Measures}

The performance measures are used to compare the performance of various algorithms against one another. In previous face detection methods, two performance measures were used. These are the detection rate and false detection rate. However, these measures are 
not clearly described in most systems as shown in a previous survey [19]. Therefore, it is difficult to evaluate system performance. Moreover, a false detection rate does not explain very well whether it is false positive or false negative. For this reason, we propose the use of the receiver operating characteristic (ROC) curve and other common metrics as our classification performance measures for our face detection method. The ROC curve and other common metrics will be briefly discussed in the following subsections.

\subsubsection{Receiver Operating Characteristic Curve}

A receiver operating characteristic (ROC) curve is a technique for visualizing, organizing, and selecting classifiers based on their classification performance. The ROC curve has been used in many fields such as signal detection theory [11], behavior diagnostic systems [31], and machine learning [27]. The ROC curve has nice properties that make it very useful and easy to work with. To provide some background about ROC curve and some common metrics, we begin our discussion by considering the two class classification problem. Two class classification is a mapping that maps any instance $I$ of the test data to one element of set $\{\mathbf{p}, \mathbf{n}\}$ of positive and negative classes. In other words, given a classifier and an instance, there are four possible outcomes. These are defined as:

- True positive (TP) is a case when an instance is positive and is classified as positive.

- False positive (FP) is a case when an instance is negative and is classified as positive.

- True negative (TN) is a case when an instance is negative and is classified as negative.

- False negative (FN) is a case when an instance is positive and is classified as negative.

These outcomes can be used to construct a confusion matrix 3.1 for a given classifier and a set of test data. In order to differentiate between the actual class and predicted class, we use the label $\mathbf{Y}, \mathbf{N}$ for class prediction produced by a classifier. 
Table 3.1: Confusion Matrix of an ROC curve

\begin{tabular}{|c|c|c|}
\hline Hypothesis class & True positive class $\mathbf{p}$ & True negative class $\mathbf{n}$ \\
\hline Positive class $\mathbf{Y}$ & Number of $\mathbf{T P}$ & Number of FP \\
\hline Negative class $\mathbf{N}$ & Number of $\mathbf{F N}$ & Number of $\mathbf{T N}$ \\
\hline Column Total & $\mathrm{P}$ & $\mathrm{N}$ \\
\hline
\end{tabular}

Let $\mathrm{N}$ denote total number of negative instances and $\mathrm{P}$ denote the total number of positive instances. The following common metrics can be defined or calculated from the confusion matrix in table 3.1 as follows:

- True positive rate (tp rate) $\approx \frac{T P}{P}$

- False positive rate (fp rate) $\approx \frac{F P}{N}$

- Hit rate $=$ Recall $=$ Sensitivity $=$ True positive rate

A ROC curve is a two-dimenentional graph. It is constructed by plotting the tp rate on the $\mathrm{Y}$ axis and the $\mathbf{f p}$ rate on the $\mathrm{X}$ axis. An $\mathrm{ROC}$ curve is the result of varying the threshold of a classifier to produce a binary classifier. For example, if a classifier has an output above a certain threshold, the classifier produces a $\mathbf{Y}$, otherwise, it produces a $\mathbf{N}$. Each threshold value produces a different confusion matrix and results in a different ROC point. An ROC shows the tradeoffs between the true positive rate and false positive rate at different thresholds values. These measures are the most important measures of system effectiveness. The ROC curve allows us to visualize the whole system's performance at different thresholds instead of a single point for a particular threshold. In the case of a face detection application, a good system should have a high true positive rate but also have a low false positive rate. 


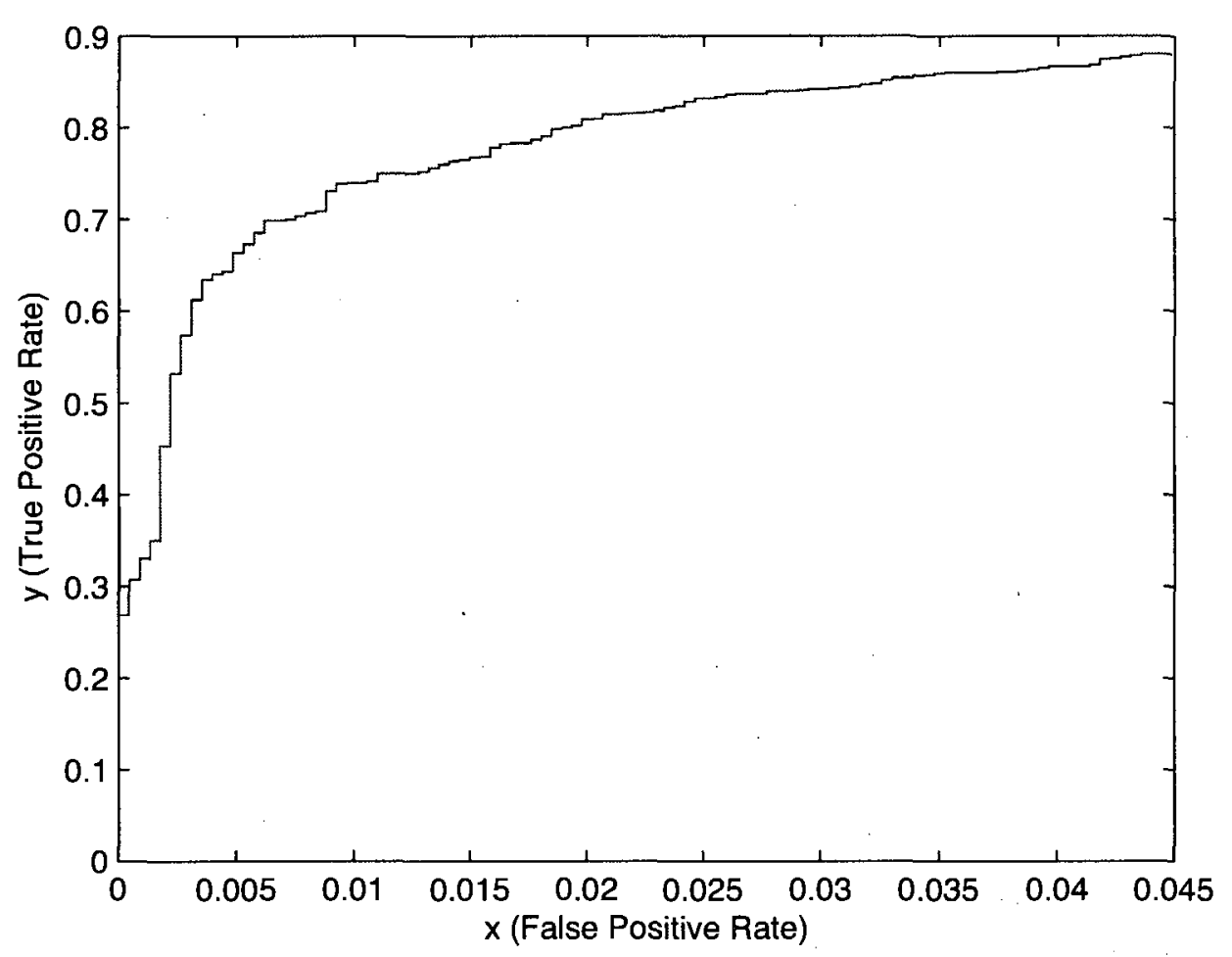

Figure 3.1: An example of an ROC curve

Table 3.2: Confusion Matrix of the ROC curve (figure 3.1)

\begin{tabular}{|c|c|c|}
\hline Hypothesis class & $\mathbf{p}$ & $\mathbf{n}$ \\
\hline $\mathbf{Y}$ & $\mathrm{TP}=1069$ & $\mathrm{FP}=102$ \\
\hline $\mathbf{N}$ & $\mathrm{FN}=145$ & $\mathrm{TN}=2171$ \\
\hline Column Total & $\mathrm{P}=1214$ & $\mathrm{~N}=2273$ \\
\hline \multicolumn{2}{|c|}{ Performance Metrics } \\
\hline TP rate $=88.056013 \%$ & TN rate $=95.512538 \%$ & \\
\hline FP rate $=4.487462 \%$ & FN rate $=11.943987 \%$ & \\
\hline
\end{tabular}




\subsubsection{Evaluation Protocol}

Evaluating previous appearance-based methods for face detection is a very challenging task because many systems use different constraints, different training sets, test sets, and only a few of them tested on the same test sets. Moreover, it is very difficult to obtain the training sets, and test sets. To ensure a fair evaluation of face detection methods, we define our evaluation protocol as follows:

1. Both methods must use the same constraints, assumptions, and data sets.

2. The same performance measures, ROC curve and other metrics must be used.

If both systems have the same performance, the simpler system will be chosen as the superior face detection method.

\subsubsection{Testing and Assumptions}

The purpose of testing is to find the strengths and weaknesses of a method. The appearancebased methods rely heavily on the assumptions and constraints made in the training data sets. In most of the discussed techniques, the constraints and assumptions are often not provided. We find that it is extremely important to have the constraints and assumptions clearly defined. Not only does it allow us to evaluate the systems better but also allows us to see the effectiveness of testing procedures. In order to construct our system constraints and assumptions, we use the following definitions:

- Face orientation: Face images directly vary for different rotations about the camera's optical axis.

- Occlusion: Faces may be partially occluded by other objects.

- Pose: The images of a face vary due to the relative camera-face pose (frontal, 45 degree, profile, upside down), and some facial features such as an eye or a nose may become partially or wholly occluded. 
- Face components: Facial features such as moles, beards, mustaches, and glasses may or may not be present.

- Facial expression: The face is directly affected by a person's moods.

Our system is designed to solve a specific face detection problem with the following assumptions and constraints:

- Our face detection system deals only with frontal face orientation and frontal pose with some degrees of variation of rotation and zoom.

- Our system solves slight occlusion cases.

- Our system solves face images with components such as beards, molds and facial expressions.

- Lighting is evenly distributed in the image.

- Our system uses the MIT data sets [20] and our new data sets.

- The variations of face images and the system ability to detect faces are based on the data sets.

\subsubsection{Analysis of the face data sets}

Data plays an important role in appearance-based face detection methods. A good data set should capture meaningful features of face and non-face structures. It sliould test the strengths and weaknesses of a technique. The recent appearance-based face detection methods [19] reported to have very good detection rates around $90 \%$ to $98 \%$. This poses a question as to whether the face detection problem is an easy problem or have we not looked into the right problems. For examples, an easy test set should result in high detection rates. Therefore, in this section, our motivation is to examine and analyze the current MIT data sets [20] and to look at the problems associated with them. Another motivation is that 
we believe that for appearance-based face detection methods, the data sets must ask an appropriate question and demand a certain level of ability of the detection technique. For example, if we use neural network methods to detect a face in a simple black and white background, it would defeat the purpose of using neural networks. Perhaps, a knowledgebased face detection method is more suitable. In order to analyze the MIT data sets, we will use the common grey scale feature to examine the data sets. Due to the fact that the grey scale feature has high dimensionality, we use the perceptron [24] to examine the data sets [20]. The perceptron will allow us to detect whether a data set is linearly or non-linearly separable. This property is very important in machine learning because it allows us to understand the problem associated with the data sets. For example, if a data set is linearly separable, it is always easier to solve than the case of non-linearly separable data set. Before we proceed with our analysis, we provide a description of the MIT data sets [20]. They are obtained from the MIT facial detection group [20]. There are 2 data sets: training set and test set. The training set consists of 2429 face images and 4548 non-face images. The test set has 472 face images and 23573 non-face images. Each image has the size of $19 \times 19$ pixels. These sets of images are resized to $20 \times 20$ pixel images because our texture features are easier to work with evenly sized blocks. We test the training data set and test set using the perceptron model [24]. Due to the imbalanced test set, we manually added an appropriate number of face images from the original test set to obtain a balanced test set. We trained the perceptron with the MIT data sets. The performance metrics of the perceptron for the training set and test set after 2000 epoches are shown in table 3.3 and table 3.4 respectfully. The results show that the training set and test set are linearly separable.

Upon examining the MIT data sets [20], we see that there are large differences between face images and non-face images. Therefore, high detection rates can be expected. For the test set, this is not a difficult test set. The reason for the perceptron to have a TP rate of $95 \%$ in table 3.4 is that the test set has around $10 \%$ of vague face patterns in the face test set. These face patterns (figure 3.2) act like outliers that make it difficult for the perceptron 
Table 3.3: Confusion Matrix and Performance Metrics of MIT training set [20]

\begin{tabular}{|c|c|c|}
\hline Hypothesis class & $\mathbf{p}$ & $\mathbf{n}$ \\
\hline $\mathbf{Y}$ & $\mathrm{TP}=2379$ & $\mathrm{FP}=57$ \\
\hline $\mathbf{N}$ & $\mathrm{FN}=50$ & $\mathrm{TN}=4491$ \\
\hline Column Total & $\mathrm{P}=2429$ & $\mathrm{~N}=4548$ \\
\hline \multicolumn{2}{|c|}{ Performance Metrics } \\
\hline TP rate $=97.941540 \%$ & TN rate $=98.746702 \%$ & \\
\hline FP rate $=1.253298 \%$ & FN rate $=2.058460 \%$ & \\
\hline
\end{tabular}

Table 3.4: Confusion Matrix and Performance Metrics of MIT test set [20]

\begin{tabular}{|c|c|c|}
\hline Hypothesis class & $\mathbf{p}$ & $\mathbf{n}$ \\
\hline $\mathrm{Y}$ & $\mathrm{TP}=22001$ & $\mathrm{FP}=3661$ \\
\hline $\mathrm{N}$ & $\mathrm{FN}=1127$ & $\mathrm{TN}=19912$ \\
\hline Column Total & $\mathrm{P}=23128$ & $\mathrm{~N}=23573$ \\
\hline \multicolumn{2}{|c|}{ Performance Metrics } \\
\hline TP rate $=95.127119 \%$ & TN rate $=84.469520 \%$ & \\
\hline FP rate $=15.530480 \%$ & FN rate $=4.872881 \%$ & \\
\hline
\end{tabular}

to separate between face and non-face patterns. In fact, many of these outliers are not face images if manually inspected with the naked eye.

Our experimental perceptron result gives us two important implications about the MIT data sets [20].

- Both training set and test set are easy to separate.

- No further improvement can be made by using this data set.

For these reasons, we will create our new data sets. We provide a full descriptions of our data sets in the below experimental design subsection. 


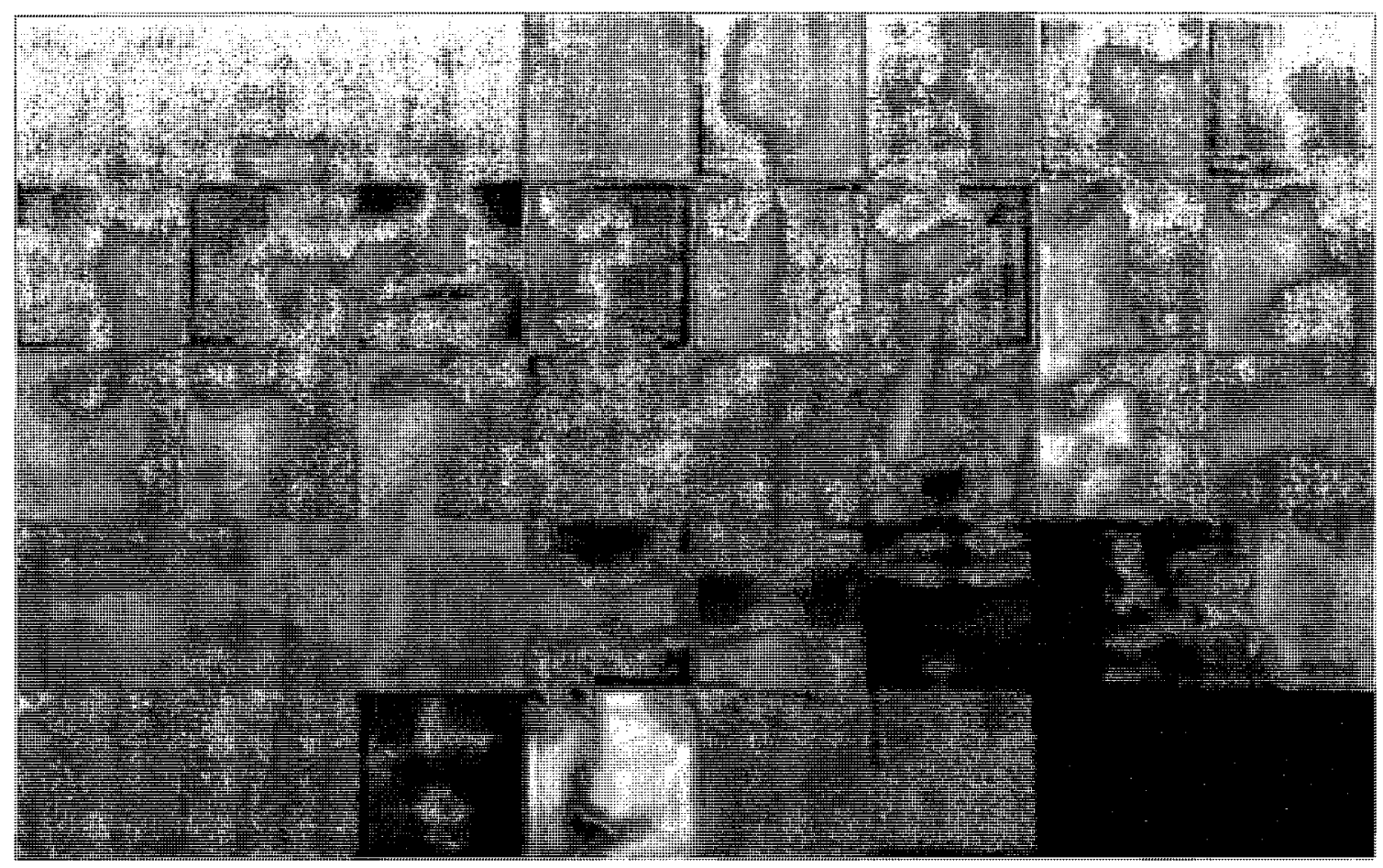

Figure 3.2: Outliners in the face test set of data set [20]

\subsubsection{System design}

Our system design is based on the design of the previous methods such as the method in [13]. Instead of using a multilayer neural network, we use SVM as our main classifier to classify face and non-face patterns. Our system consists of three subsystems: sampling and preprocessing subsystem, texture feature extraction subsystem and SVM subsystem. Like the previous method [13], an input image is sampled and preprocessed with a histogram equalization method. This eliminates the large variations in image contrasts and brightness. We assume that image is sampled by feeding an individual image instead of scanning the windows across a large image. Therefore, the scanning process is not employed in our method. Because the texture correlation feature is easy to extract with images of even block size, we resize all our data sets to $20 \times 20$ pixel images. After first subsystem is done, the image is passed to the second subsystem which is responsible for extracting texture 
correlation features. The newly extracted texture feature vector then is fed into the third SVM subsystem to perform classification. The overall system layout is shown in figure 3.3

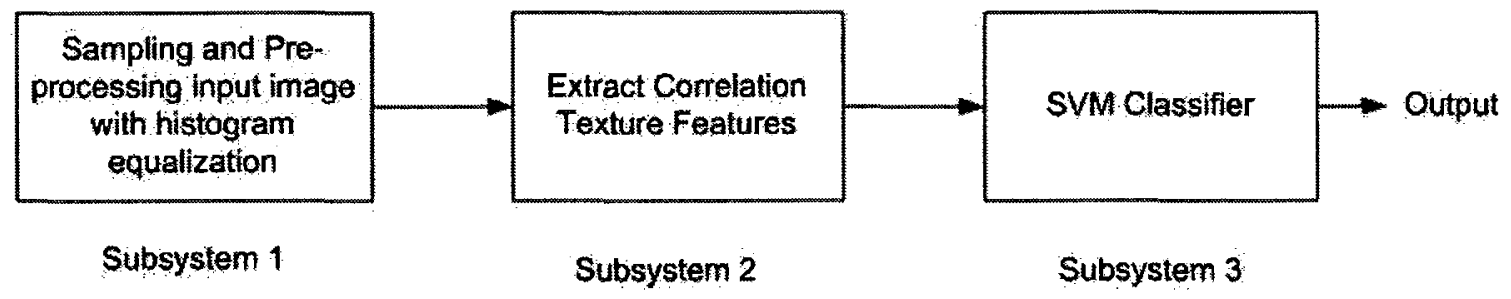

Figure 3.3: Our system design layout

In subsystem 2, the correlation features are calculated for the whole image and each section $\mathrm{A}, \mathrm{B}, \mathrm{C}, \mathrm{D}$, and $\mathrm{E}$ in figure 3.4. The horizontal and vertical offsets are used. To save computing time, region $\mathrm{A}, \mathrm{B}$, and $\mathrm{C}$ use only horizontal offsets whereas region $\mathrm{D}$ and E use vertical offsets. Both vertical and horizontal offsets are used to calculate the whole image correlation features. The result of all region correlation features can be combined into a vector with the size of 133 correlation feature elements.

\subsubsection{Experimental design}

Many appearance-based face detection systems have used extremely large training sets. Having a large training set does not guarantee a solution to the face detection problem because the face image distribution is unknown. Due to this reason, general face detection tasks are often intractable. Therefore, our face detection problem focuses on solving a specific task. Our motivation for this section is to ask a very simple question: what are the disadvantages of grey scale features and texture correlation features? In order to understanding the weaknesses of both features, we start our discussion by looking at the traditional two-class classification problem. Traditional two-class classification problems refer to the assignment of instances into two predefined classes. Such problems often arise in many application fields. In the face detection application, two distinct problems as in figure 3.6 normally arise. 

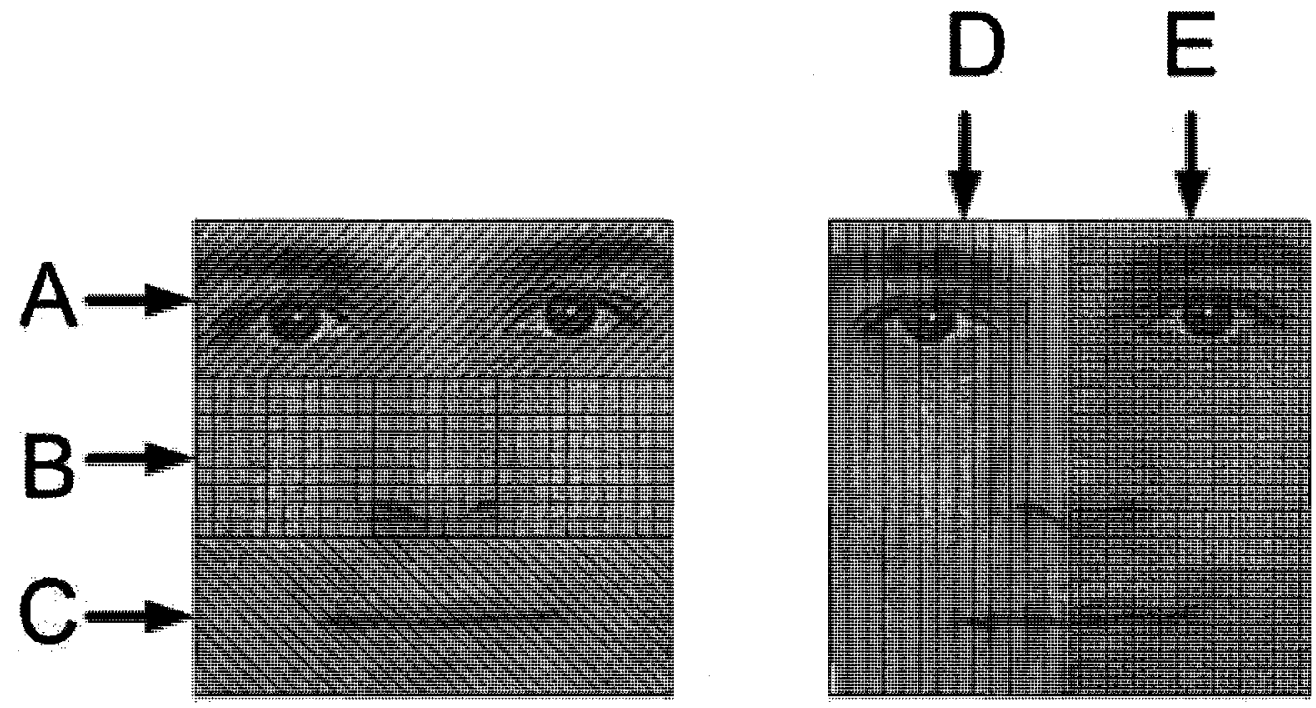

Figure 3.4: In subsystem 2, an input image is divided into regions $\mathrm{A}, \mathrm{B}, \mathrm{C}, \mathrm{D}$, and $\mathrm{E}$ for the texture correlation calculation

- The first problem is a two-class classification problem of face images and undefined non-face images.

- The second problem is a two-class classification problem of well-defined face images and well-defined non-face images.

Most appearance-based face detection methods fall under the first classification problem. The problems that are associated with it are the fact that face images might be relatively easy to define, however, it is difficult to define non-face images. This is because non-face images can be anything that is not a face image. They can be:

- Images with completely black or white backgrounds.

- Images with pixels of random intensity.

- Images of scenery. 


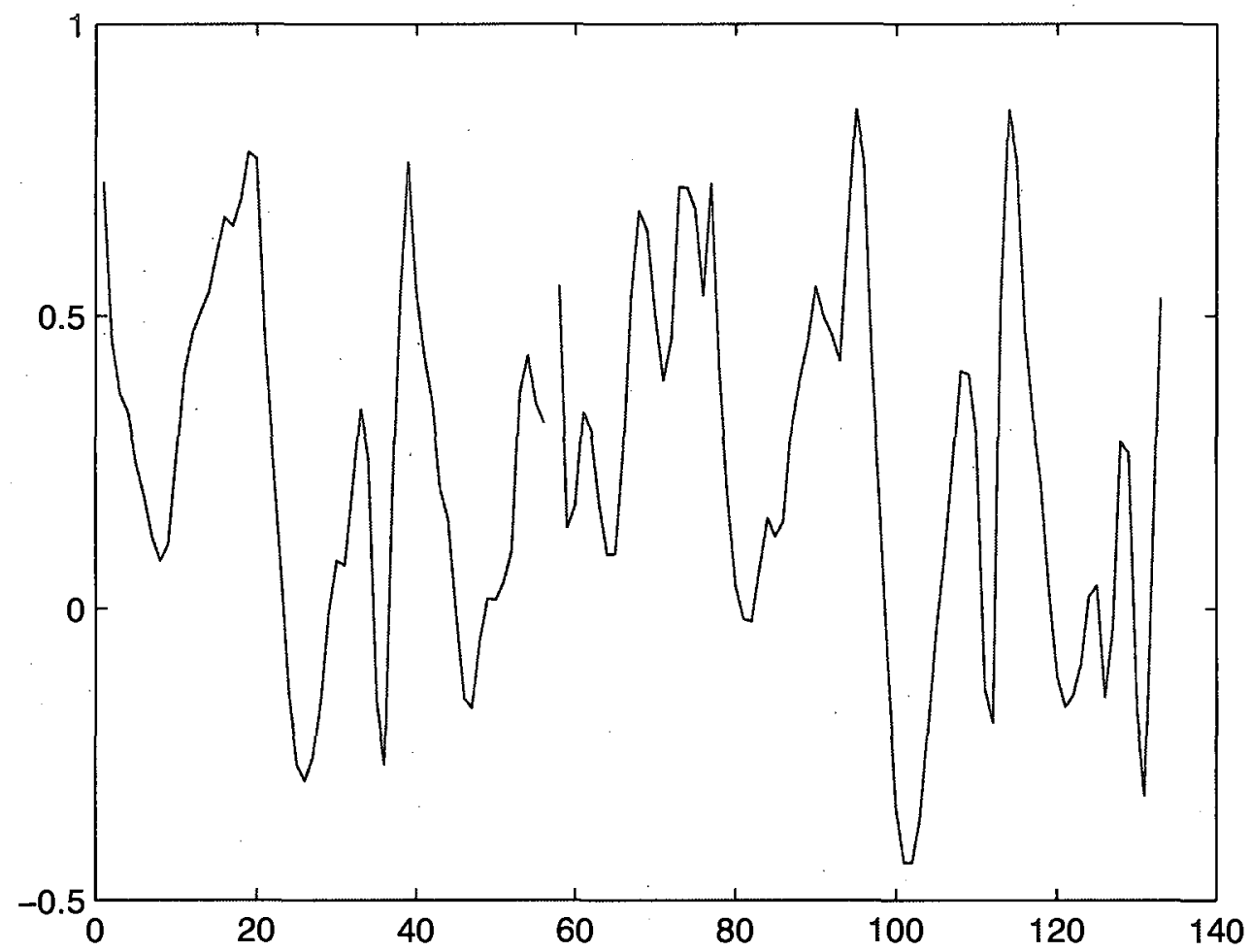

Figure 3.5: A face image is transformed into the texture correlation feature space

- Images of distorted faces, etcetera.

These non-face images might create an easy or challenging problem for a face detection application. Moreover, testing the system with undefined non-face images is a very difficult task. For examples, most appearance-based face detection methods use the common method of generating and selecting training examples from Rowley et al. [13] and Sung and Poggio [30]. This can be described as follows:

1. For face images, 15 face images are generated from an original face image in the training set by rotating them about their central point up to 10 degrees, scaling between 90 to 110 percent, and minoring. This allows the system to deal with more 


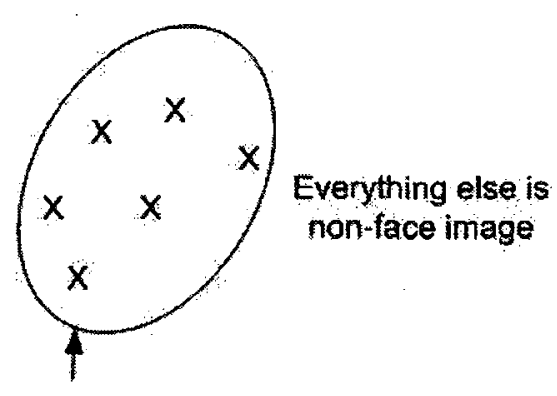

Face images

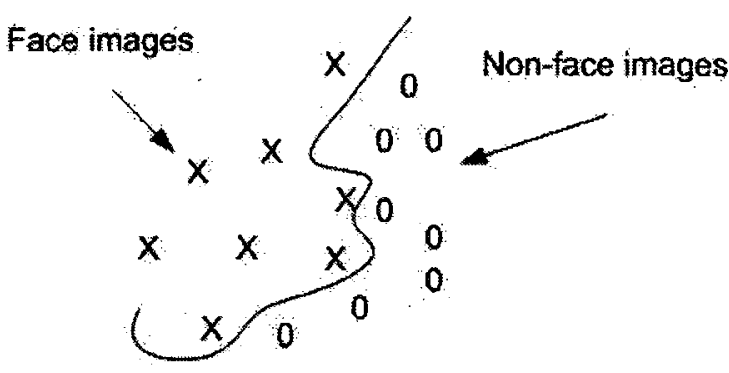

(a) (b)

Figure 3.6: Two-class classification problems. (a)two-class classification problem of face images and undefined non-face images, (b) two-class classification problem of face images and well-defined non-face images.

variations of face images.

2. For non-face images,

(a) Create an initial set of non-face images by generating 1000 images with random pixel intensities.

(b) Train the classifier to produce an output of 1 for the face examples, and - 1 for the non-face examples.

(c) Run the system on an image of scenery which has no faces. Collect sub-images in which the network incorrectly identifies a face. Add them into the training set as negative examples. Go to step (b).

This method of generating and selecting training examples gives a definition of face and non-face images. The definition of the face in this case is the frontal face image of the original data set with some variations of rotation and scaling. However, the definition of non-face is difficult to define and to reconstruct because it relies on images of scenery, the initial random intensities of images and, the large number of non-face images. It poses a 
question of how can we sample non-face images correctly or adequately since the space of non-face images is very large? This sampling process of an ill-defined non-face image space leads to difficulty in evaluating system performance. You can have a very high detection rate or very low detection rate. It depends on the construction of the data sets and how difficult it is to separate the face and non-face patterns. Unfortunately, Rowley's database of images ia no longer available. Therefore we were not able to examine its non-face images.

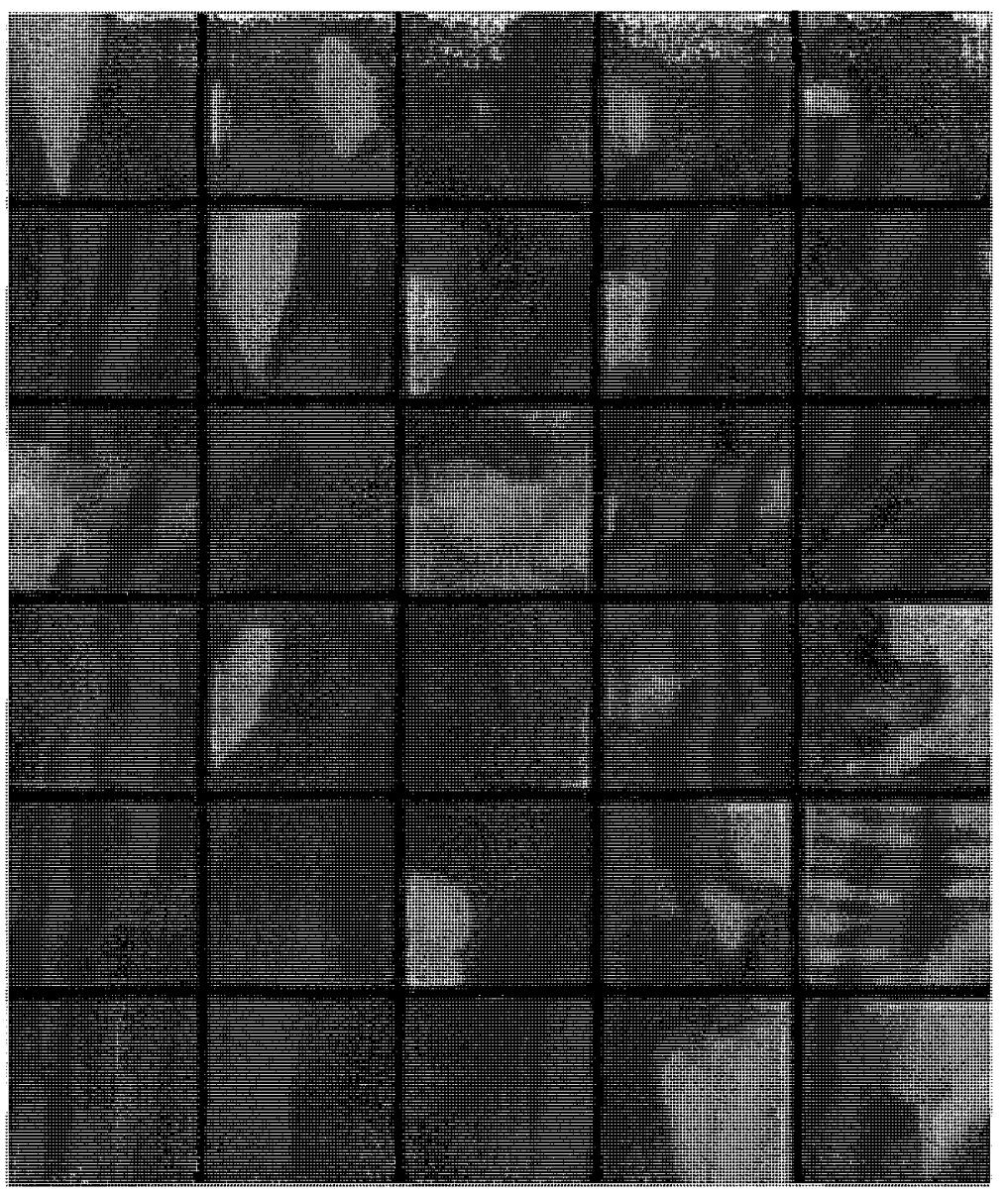

Figure 3.7: Non-face images of MIT CBCL test set [20] 


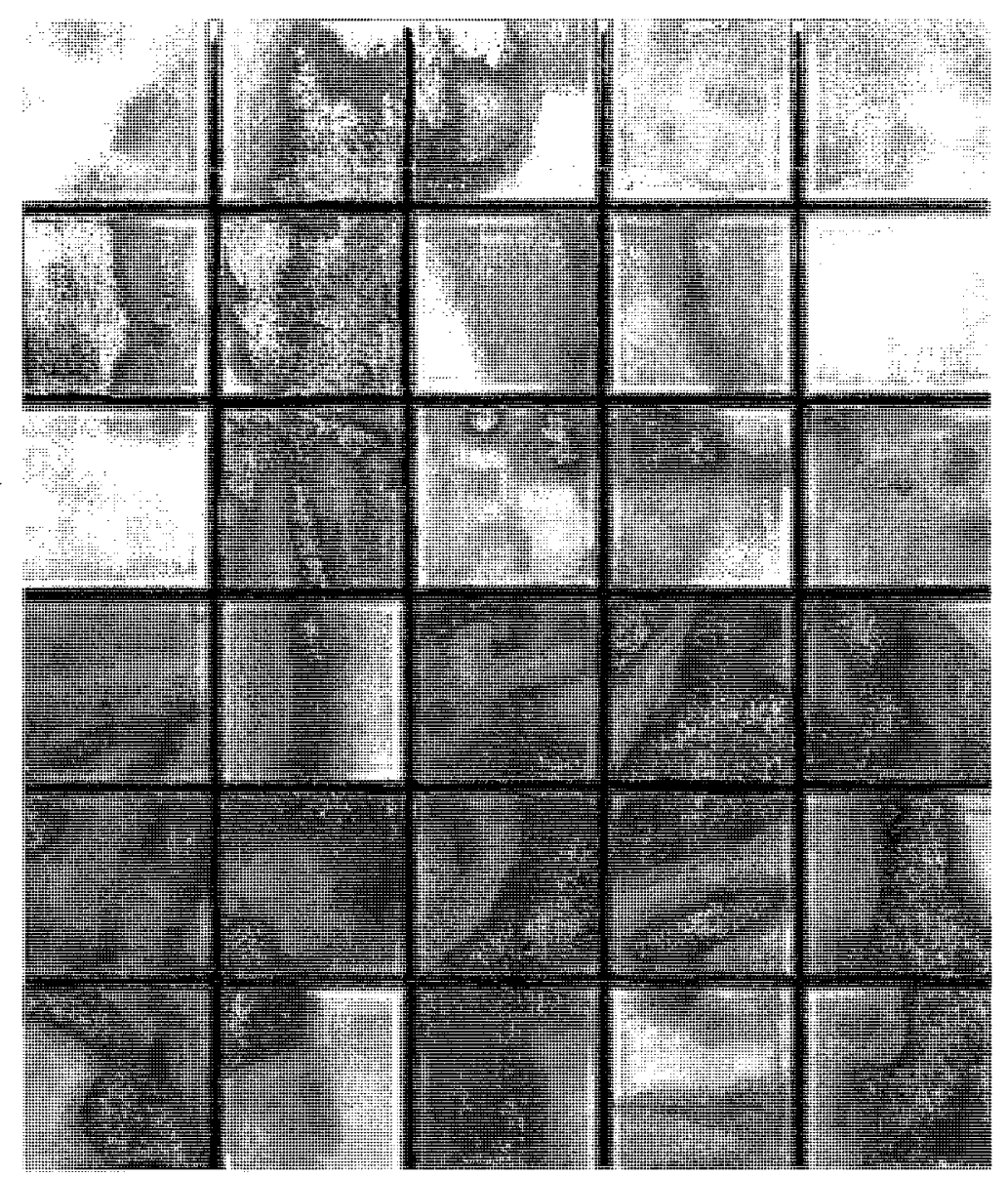

Figure 3.8: Non-face images of MIT CBCL test set [20]

To show the limitation of the grey scale feature and the texture correlation feature, we examine a second classification problem. We evaluate the classification problem of welldefined face images and well-defined non-face images. We define our face images as the frontal face images from the MIT data set [20] (with the exception in our data set 3 that has face images from the MIT data set and our scrambled face images). We define our nonface images by the scrambling of face images until they are no longer face images. We create 3 different data sets. Our first data set is described in figure 3.9. For the first data set, fifty training sets are created by combining set $\mathrm{A}$ with set $\mathrm{C} 1, \mathrm{C} 2, \ldots$, and C50. Fifty test sets are created by combining set B with set D1,D2,... and D50. Similarly, we create our second data 
set by scrambling the rows and columns of face images to create non-face images similar to the process described in figure 3.10. Twenty training sets and twenty test sets are created. In our first two data sets, we test and examine the affects of scrambling pixels and columns and rows of non-face images on our system classification performance. Though these two tests provide the trend of our system classification behavior with scrambling levels. it does not test the cases where face images are slightly distorted included in our face data sets. In order to test this case, we create our data set $\mathbf{3}$. Our data set $\mathbf{3}$ can be constructed by:

1. Create data set 1 as describe in figure 3.9 .

2. Choose a scramble level $\Delta$ where face images are distorted into non-face images.

3. Create data set 3 from data set 1 by:

- For both training set and test set, combine scramble images at scramble level $\Delta-3$ with face images of data set 1 (MIT face data set) to create face data sets and combine scramble images at level $\Delta+3$ and level $\Delta+6$ to create non-face images.

In the our first data set, each training set has 1215 face images and 1215 non-face images. Each test set has 1214 face images and 1214 non-face images. In our second data set, each training set has 1215 face images and 2430 non-face images. Each test set has 1215 face images and 2430 non-face images. In our third data set, the training set has 2430 face inages and 2430 non-face images. The test set has 2428 face images and 2428 non-face images. To avoid the confusion, we denote our first data set as data set 1, our second data set as data set $\mathbf{2}$, and our third data set as data set $\mathbf{3}$.

Our motivation for creating non-face images by scrambling face images are two folds. First, we want to test how well the grey scale feature and the texture correlation feature can detect non-face patterns that are closer to face patterns. Second, it will help our system to deal with non-face patterns that are closer to face images. In our data set 3 , we add slightly distorted face images into our face data sets. It will allow us to test the more challenging case for our system as compared to data set 1 and 2 . 


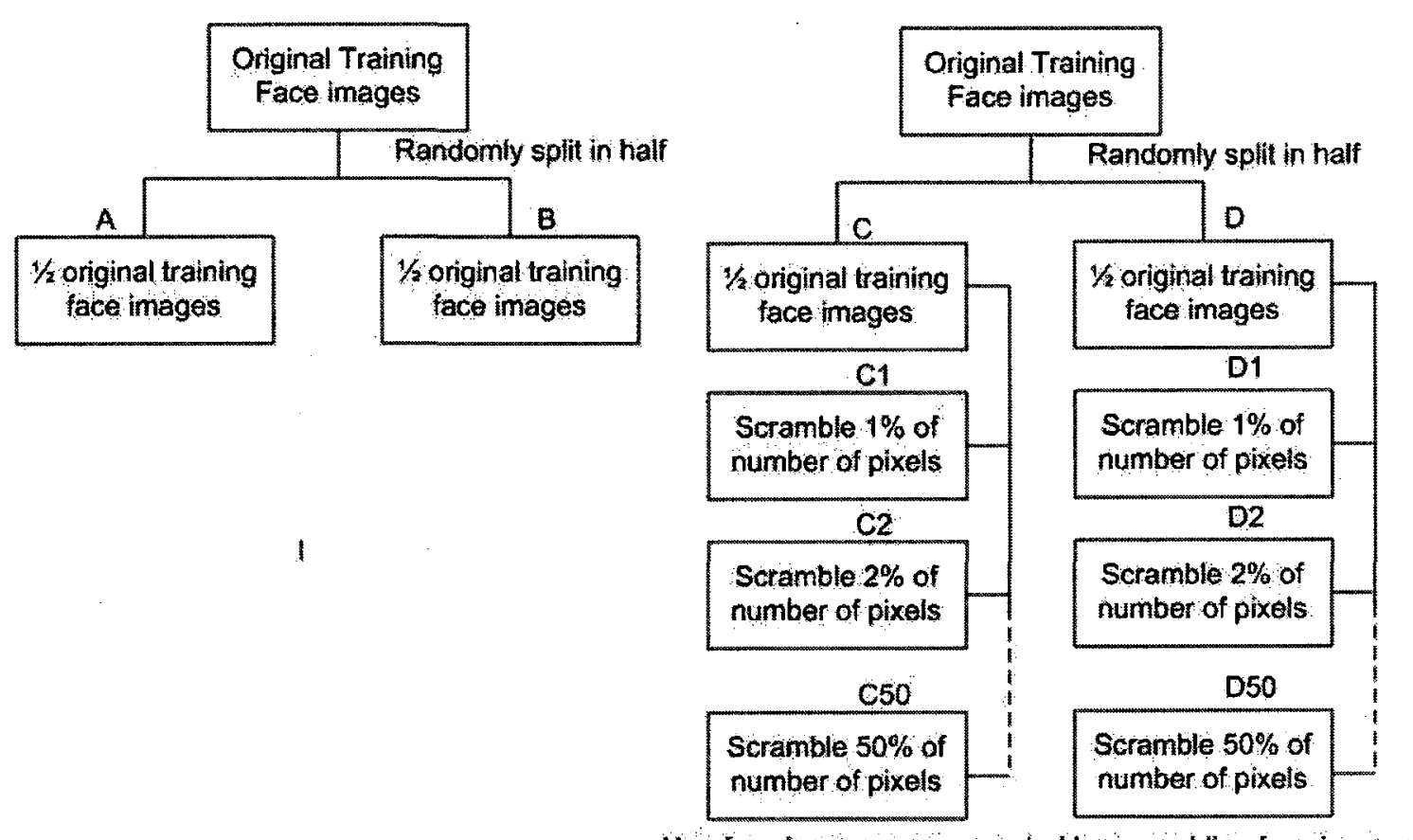

Non-face images are generated by scrambling face images.

Figure 3.9: Our first data set

We implement 72 systems out of 3 data sets for each feature using SVM as the main classifier. Each is trained with the 2nd polynomial kernel of SVMLight package [34]. We will show our experimental results in the next chapter.

\subsubsection{Quantization level and its affect on SVM for texture features}

Quantization level is used to construct the GLCM matrix which is used to calculate the correlation feature or Haralick texture feature. Quantization level is very important to investigate because it will affect the applicability of texture features. Choosing a wrong quantization level can lead to very long extraction times or can have adverse affects on the classification performance. Therefore, the ideal quantization level should have the lowest extraction time while having optimal classification performance. To choose a quantization level for our texture correlation feature, we ran our system using various quantization levels. 


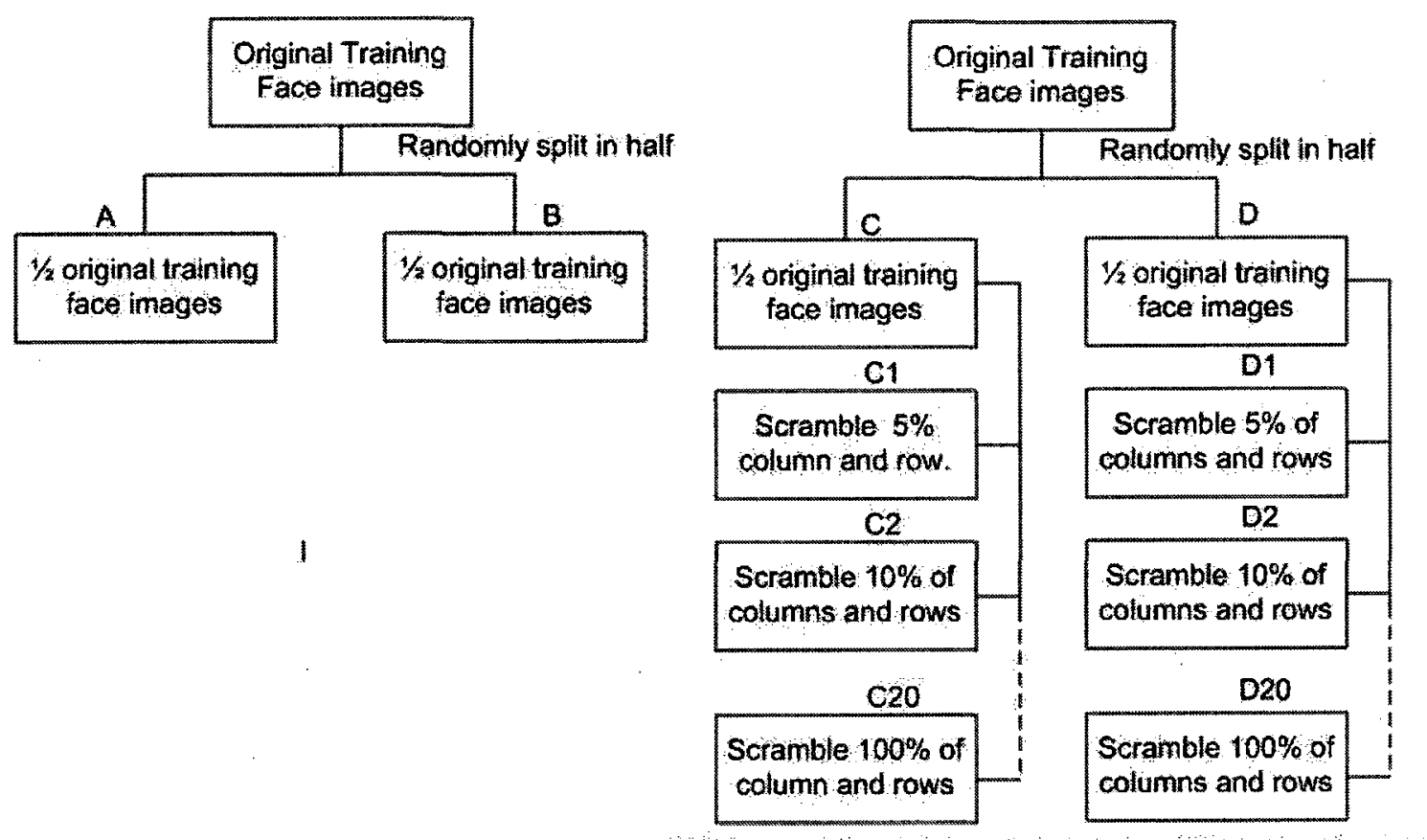

Non face images are generated by scrambling face images.

Figure 3.10: Our second data set

First, we measured the feature extraction time of 288 images for our texture correlation feature with various quantization levesl and grey scale features. Second, we set up a system that uses our data sets 2 at scramble level 10 with various quantization levels. Specifically, we create 8 SVM systems with quantization level $2,4,8,16,32,64,128$, and 255 to test the quantization level effects on the SVM. The result of these experiment will allow us to choose an ideal quantization level for our system.

\subsubsection{Complexity of Our Method}

We analyze the complexity of our system. Our system consists of 3 subsystems. The complexity of our system can be calculated as $O_{\text {system }}(s 1+s 2+s 3)$ where s1, $\mathrm{s} 2$, and s3 are the complexity of subsystem 1, subsystem 2 and subsystem 3 . In subsystem 1 , the complexity can be calculated as $O_{h e q}=n * m(t+k)$ [28] where heq stands for the histogram 
equalization for an image with size of $\mathrm{n} \mathrm{x} \mathrm{m} \mathrm{with} \mathrm{range} \mathrm{of} \mathrm{intensity} \mathrm{levels} \mathrm{k}$ and contextual region size $\mathrm{t} \times \mathrm{t}$. In subsystem 2 , the complexity can be obtained in several steps. The first step is to calculate the cost of building GLCM matrices which is $O_{\text {glcm }}=n * m$ for image with size of $\mathrm{n} \times \mathrm{m}$ pixels. The second step is to compute some statistical properties such as means and standard deviations for texture correlation features. In order to show the complexity of correlation feature, we reproduce the correlation formulae here for convenience

$$
\begin{gathered}
f_{3}=\frac{\sum_{i} \sum_{j}(i j) p(i, j)-\mu_{x} \mu_{y}}{\sigma_{x} \sigma_{y}} \\
R=\sum_{i=1}^{N g} \sum_{j=1}^{N g} P(i, j) \\
p(i, j)=\frac{P(i, j)}{R} \\
p_{x}(i)=\sum_{j=1}^{N g} p(i, j) \\
p_{y}(j)=\sum_{i=1}^{N g} p(i, j)
\end{gathered}
$$

where $N g$ is grey level. To calculate the complexity of correlation features, we need to calculate the complexity of $p(i, j)$ which is $\left.O_{p(i, j}\right)=N g^{2}$, the complexity of $\mu_{x} \mu_{y}$ which is $O_{\text {mean }}=N g$ and $\sigma_{x} \sigma_{y}$ which is $O_{\text {deviation }}=N g$ where means and deviations $\mu_{x}, \mu_{y}, \sigma_{x}, \sigma_{y}$ are defined as means and deviations of $p_{x}$ and $p_{y}[23]$.

From above equations, the complexity calculating texture correlation features is:

$$
\begin{gathered}
O_{c}=O_{g l c m}+O_{p(i, j)}+N g^{2}+2 * O_{\text {mean }}+2 * O_{\text {deviation }} \\
O_{c}=n * m+2 * N g^{2}+2 * O_{\text {mean }}+2 * O_{\text {deviation }} \\
O_{c}=n * m+2 * N g^{2}+4 * N g
\end{gathered}
$$


Since we vary the offsets either horizontally or vertically, the complexity of correlation features with various offset is the number of offsets multiplied with the equation (3.8) which is:

$$
O_{c o}=\text { offsets } * O_{c}
$$

In our subsystem 2, an image is divided into A, B, C, D, and E regions for texture calculation. We calculate texture correlation for whole image and texture correlation for each regions. Therefore the complexity of texture calculation of an image can be calculated using equation (3.9):

$$
O_{c o_{\text {image }}}=O_{c o_{W}}+O_{c o_{A}}+O_{c o_{B}}+O_{c o_{C}}+O_{c o_{D}}+O_{c o_{E}}
$$

The complexity of SVM is $O_{s v m}=N$ which is the cost of constructing the hyperplane by using equation (2.3) or (2.4) where $\mathrm{N}$ is the number of training samples. Normally the hyperplane is constructed in the training phase. Thus SVM complexity should be 1 for 1 image or $\mathrm{N}$ for $\mathrm{N}$ images. We now can formulate the complexity of our system.

$$
\begin{gathered}
O_{\text {system }}=O_{h e q}+O_{c o_{\text {image }}}+O_{\text {svm }} \\
O_{\text {system }}=n * m(t+k)+O_{\text {coimage }}+N
\end{gathered}
$$

\subsection{Conclusion}

In this chapter, we constructed our methodology by looking at problems in current appearancebased face detection systems. We recognized that there is a lack of uniformity in evaluation of face detection methods due to the use of different assumptions and constraints. Thus, it is difficult to evaluate system performance. We provided the solution to evaluation problems by constructing a standardized evaluation protocol using receiver operating characteristic curve and common metrics as evaluation tools. We provided an analysis of the MIT data sets. We showed that they are linearly separable. Instead of using the MIT data sets, 
we looked at a non-linearly separable case in which we defined our problem domain by examining the well-defined face images and well-defined non-face images. We provided an algorithm to create non-face images. Our data sets will allow us to compare the classification performance of the system using gray scale features and the system using texture correlation features and how well they work in separating face and non-face patterns. In the next chapter, we provide detailed experimental results of our system using our data sets for grey scale and texture correlation features. Detailed experimental results for quantization level and the affects of quantization level will be provided. 


\section{Chapter 4}

\section{Empirical Results and Analysis}

\subsection{Introduction}

In this chapter, we discuss our experimental results using the methodology described in chapter 3. We show that our definition of face and non-face patterns are significant to the face detection system because it allows our system to take into consideration non-face patterns that appear closer to face patterns. It also allows us to test the limitations of our system with grey scale and texture features. The empirical results of our system show that using the texture correlation feature and SVM might offer an effective way to alleviate the problems of grey scale features when dealing with non-face patterns similar to those in our data sets. This chapter is divided into 2 sections. The first section provides the empirical results of our system that use grey scale features and texture correlation features. The second section examines the quantization level and its effectiveness.

\subsection{Experimental results}

In the previous chapter, we discussed the two-class classification problem. We constructed our methodology by examining a classification problem of well-defined face patterns and 
well-defined non-face patterns. Our goals are not meant to declare a winner nor to provide a ultimate face detection system. Instead, we examine the strengths and weaknesses of both grey scale features and texture features so that we can apply them properly. This section is organized into 4 subsections. In the first 2 subsections, we examine the advantages and disadvantages of our system with grey scale and texture correlation features. In the last 2 subsections, we provide visual clues about our data sets and provide a comparison of our system performance of grey scale and texture correlation features.

\subsubsection{The advantages and disadvantages of grey scale features}

\subsubsection{Data set 1}

To begin our discussion of our experimental results for grey scale features, we present our experimental results for our data set 1 in figure 4.1. In order to obtain figure 4.1, we plot the TP rate and FP rate against the scramble level for grey scale features. The scramble level indicates how close the non-face images are to the face images. The higher the scramble level, the more the dissimilarity between face and non-face images. Figure 4.1 shows the trend of our system performance with the effects of scramble level. Our interest is to exam the behavior of our system at various scramble levels. It will allow us to understand the advantages and disadvantages of our grey scale system. In this section, we will discuss our system performance at scramble level 30 and above and at scramble level 22 and below. According to our results, our system with grey scale features performs well at scramble level 30 and above. We obtain a high TP rate of $97.19 \%$, a low FP rate of $0.65 \%$, FN rate of $2.80 \%$, and TN rate of $99.34 \%$.

It is important to know that in this data set, our scramble face images are distorted into non-face images at scramble level 22. At this level, facial features such as eyes, nose, and mouth are distorted. In our experiment, our system with grey scale features has a lower TP rate of $92.99 \%$ and FP rate of $2.38 \%$ at scramble level 22 . It is worth to note that a higher FN rate of $7.0 \%$ and a TN rate of $97.61 \%$ is obtained. Our system seems to misclassify 


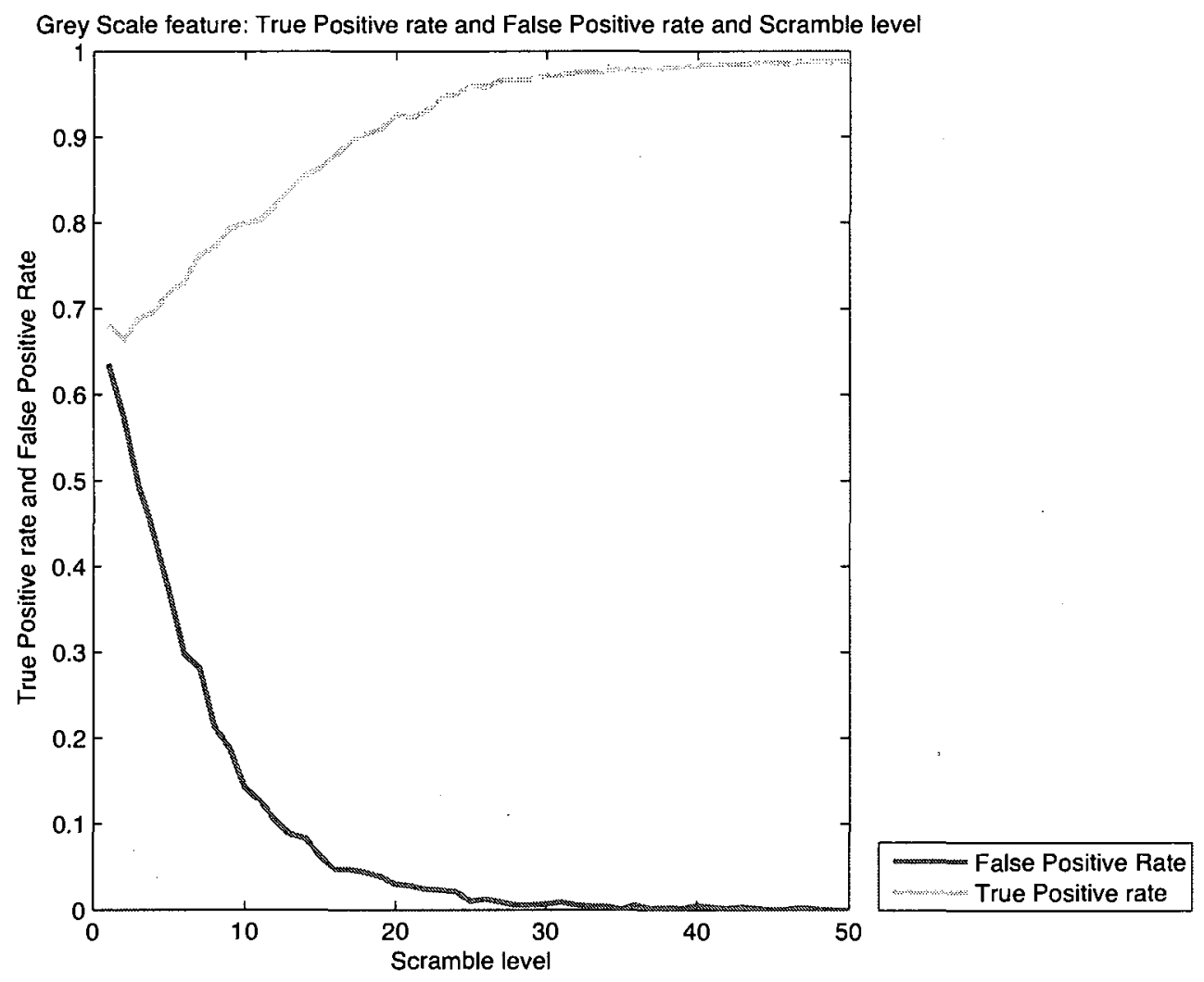

Figure 4.1: Our experimental results of our system with grey scale features for data set 1 non-face images as face images at the rate of $7 \%$. Even though the TP rate at scramble level 22 is $92 \%$, our data set 1 has only around 2450 images. A real world application where the test data set has more non-face images similar to those at scramble level 22 will have a negative impact on the system performance. It will lead to much lower TP rates. Our experimental results show that our system with grey scale features has some disadvantages in dealing with non-face images at scramble level 22. For scramble level below 22, our system behaves as expected. Due to a closeness of non-face patterns to our face patterns, our system was not be able to separate the 2 patterns. Therefore a low TP rate and a high FP rate are obtained. Detailed experimental results at scramble level 22 for grey scale features are shown in figure 4.2 and table 4.1. 
Table 4.1: Confusion Matrix of ROC curve (figure 4.2) and performance metrics for our data set 1 at scramble level 22

\begin{tabular}{|l|l|l|}
\hline \multicolumn{3}{|c|}{ Grey scale features } \\
\hline Hypothesis class & True Positive Class $\mathrm{p}$ & True Negative Class $\mathbf{n}$ \\
\hline Positive Class $\mathbf{Y}$ & $\mathrm{TP}=1129$ & $\mathrm{FP}=29$ \\
\hline Negative Class N & $\mathrm{FN}=85$ & $\mathrm{TN}=1185$ \\
\hline Column Total & $\mathrm{P}=1214$ & $\mathrm{~N}=1214$ \\
\hline \multicolumn{2}{|c|}{ Performance metrics for grey scale feature } \\
\hline TP rate $=92.998353 \%$ & TN rate $=97.611203 \%$ & \\
\hline FP rate $=2.388797 \%$ & FN rate $=7.001647 \%$ & \\
\hline
\end{tabular}

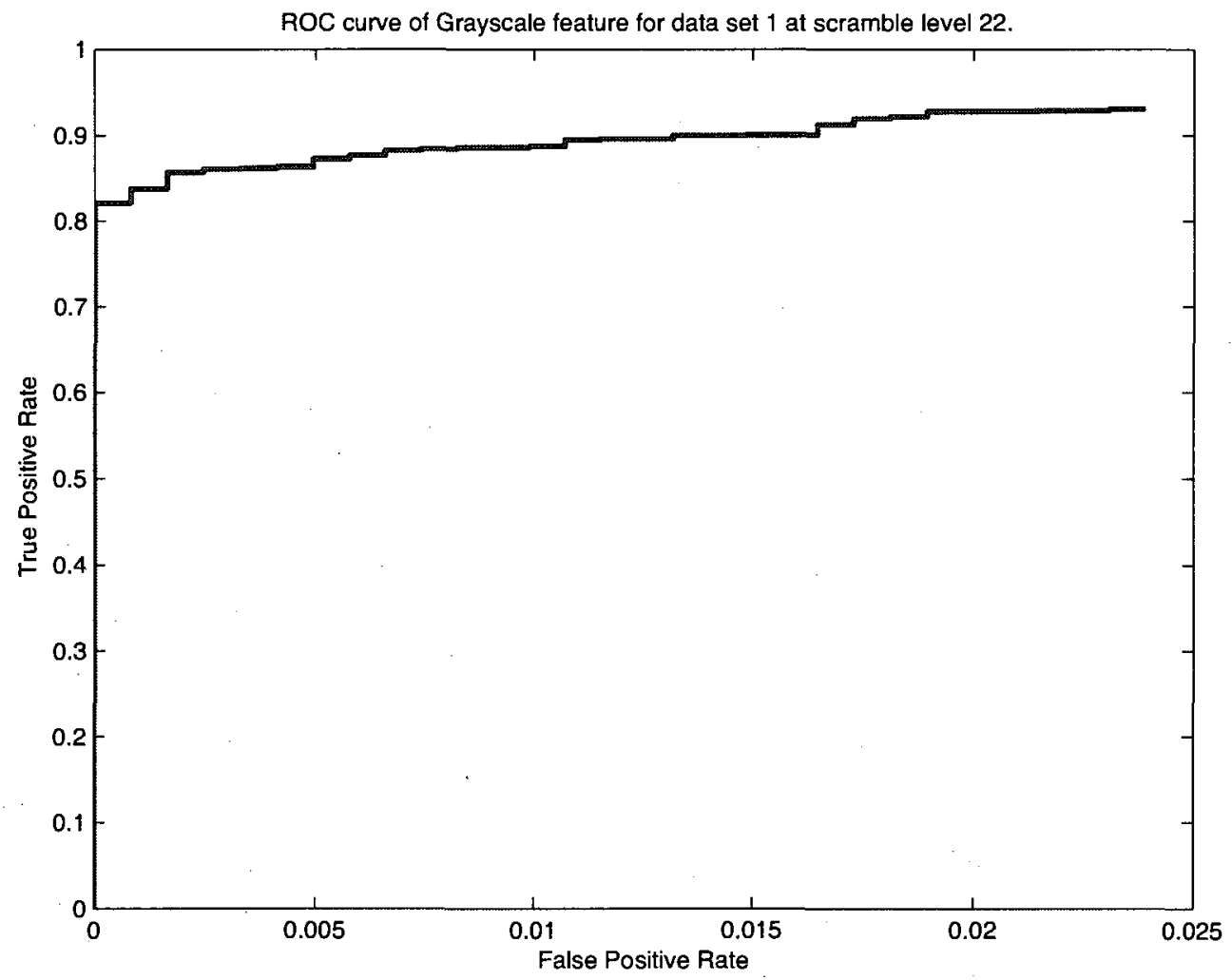

Figure 4.2: ROC curves for grey scale features for our data set 1 at scramble level 22 


\subsubsection{Data set 2}

In our data set 2 , we scramble the face images by columns and rows to obtain non-face images. The scramble levels are varied from 0 to 20 . Unlike non-face images scrambled by pixels, non-face images slightly preserve some structure of face images while facial components are distorted. The experimental results for data set 2 are shown in figure 4.3.

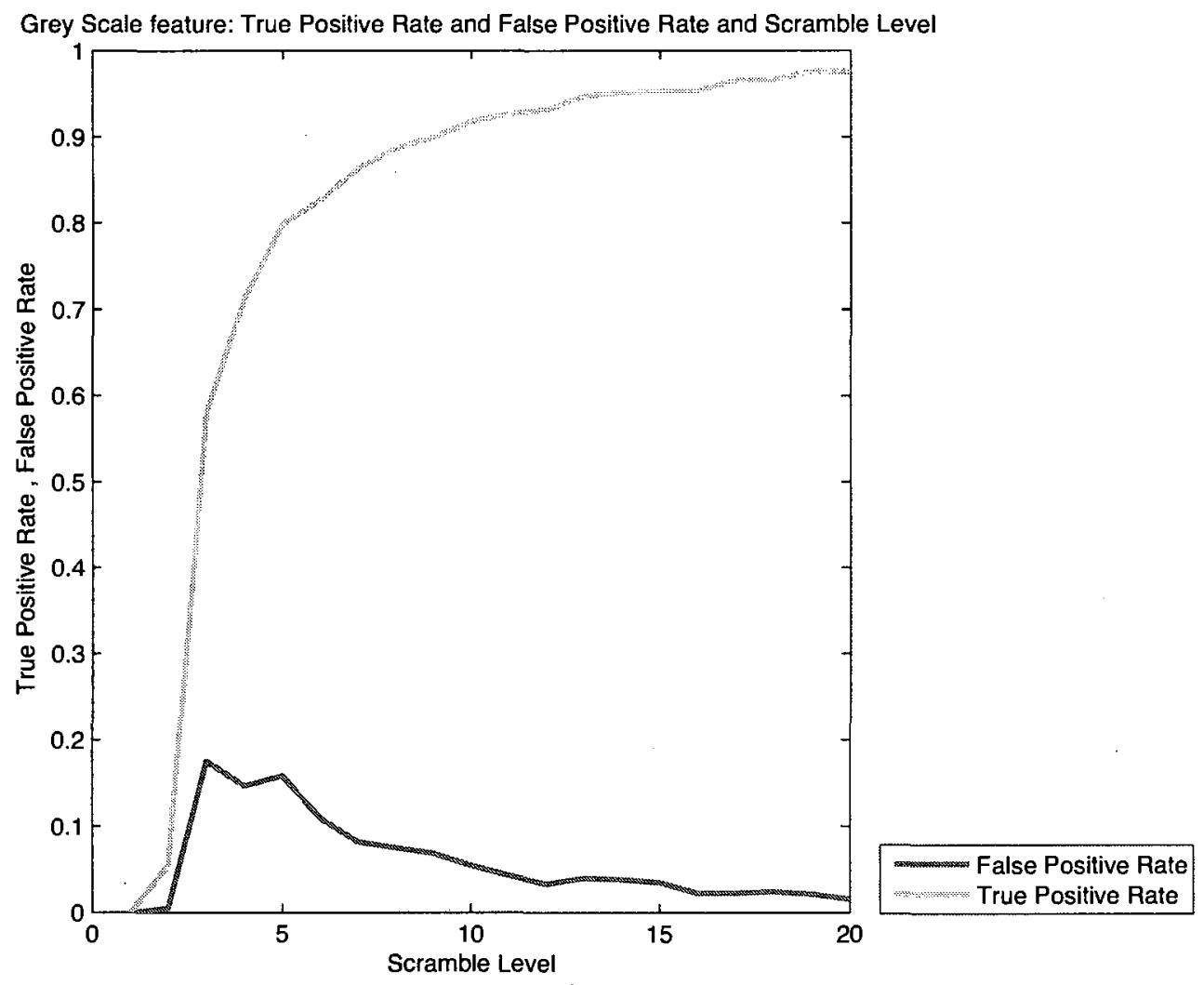

Figure 4.3: Our experimental results of our system with grey scale features for data set 2

In data set 2, our face images are distorted into non-face images at scramble level 10. However, our system performs well only at scramble level 19. We obtain a high TP rate of $97.61 \%$, a low FP rate of $2.14 \%$, a low FN rate of $2.38 \%$, and a high TN rate of $97.85 \%$. At scramble level 10 , we obtain TP rate of $91.68 \%$, FP rate of $5.43 \%$, FN rate of $8.3 \%$, TN rate of $97.28 \%$. Our system experiences a drop in performance as compared to scramble 
level 19. TP rate is lowered by nearly $6 \%$ while FP rate and FN increase to $5.43 \%$ and $8.3 \%$ respectively. At scramble levels below 10, our system TP rate decreases while FP increases. Figure 4.3 and figure 4.1 show that our system does not perform as well as with data set 1. Non-face images scrambled by columns and rows create a more challenging task for our system as compared to data set 1 due to the fact that some facial structures still remain when scrambling images by columns or rows. Detailed experimental results for our data set 2 at scramble level 10 is shown in the figure 4.4 and table 4.4 .

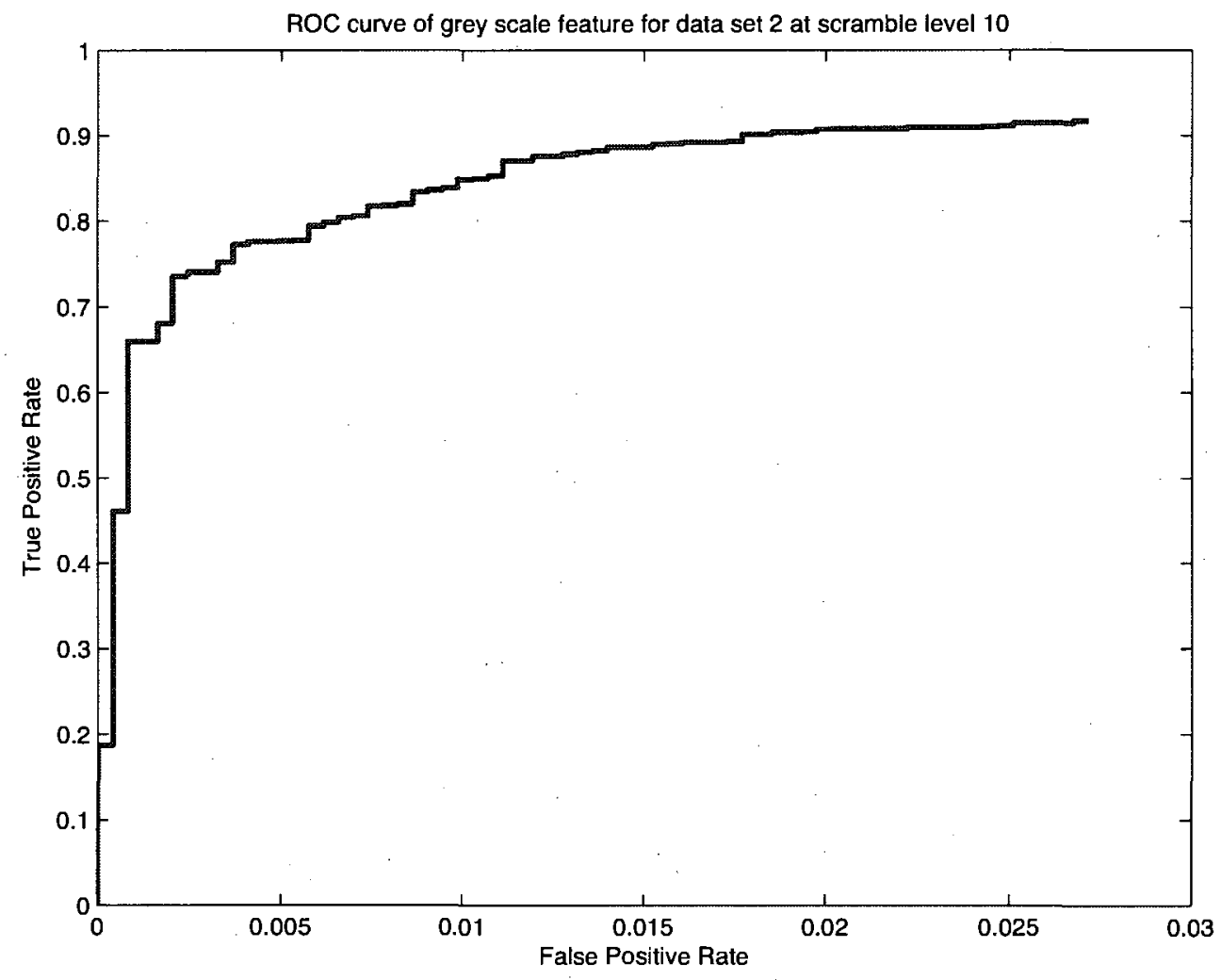

Figure 4.4: ROC curve of grey scale features for our data set 2 at scramble level 10 
Table 4.2: Confusion Matrix of ROC curves (figure 4.4) and Performance Metrics for our data set 2 at scramble level 10

\begin{tabular}{|l|l|l|}
\hline \multicolumn{3}{|c|}{ Grey scale features } \\
\hline Hypothesis class & True Positive Class $\mathbf{p}$ & True Negative Class $\mathbf{~}$ \\
\hline Positive Class $\mathbf{Y}$ & $\mathrm{TP}=1114$ & $\mathrm{FP}=66$ \\
\hline Negative Class $\mathbf{N}$ & $\mathrm{FN}=101$ & $\mathrm{TN}=2364$ \\
\hline Column Total & $\mathrm{P}=1215$ & $\mathrm{~N}=2430$ \\
\hline \multicolumn{3}{|c|}{ Performance metrics for grey scale features } \\
\hline TP rate $=91.687243 \%$ & TN rate $=97.283951 \%$ & \\
\hline FP rate $=2.716049 \%$ & FN rate $=8.312757 \%$ & \\
\hline
\end{tabular}

\subsubsection{Data set 3}

In the previous data set, we tested the face images against distorted non-face images. In this data set, we examine the effect of adding distorted face images into our data set 1 at scramble level 19. It will create more challenges to our system as compared to our previous data sets. To do so, we constructed our data set as described in chapter 3 . In our data set 1 , our face images are distorted into non-face images at scramble level 22 . To create our data set 3, we constructed our data set by adding images at scramble level 19 into our face sets for both training set and test sets and adding images at scramble level 25 and 28 to our non-face images. The experimental results are reported in figure 4.5 and in table 4.3 . 


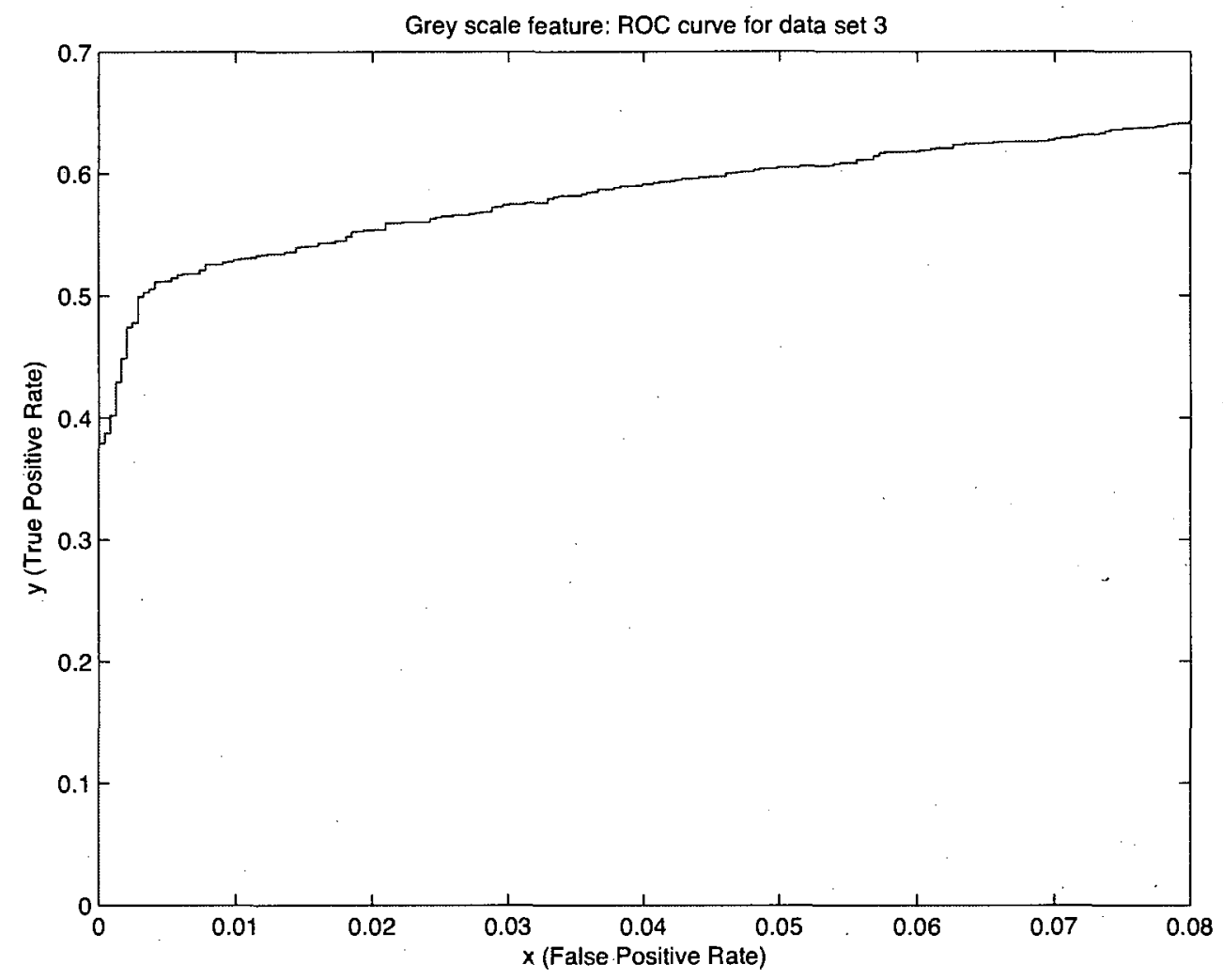

Figure 4.5: ROC curve for our system with grey scale features for our data set 3

Table 4.3: Confusion Matrix of ROC curves (figure 4.5) and Performance Metrics for our data set 3

\begin{tabular}{|l|l|l|}
\hline \multicolumn{3}{|c|}{ Grey scale features } \\
\hline Hypothesis class & True Positive Class $\mathbf{p}$ & True Negative Class $\mathbf{~}$ \\
\hline Positive Class $\mathbf{Y}$ & $\mathrm{TP}=1559$ & $\mathrm{FP}=194$ \\
\hline Negative Class N & $\mathrm{FN}=869$ & $\mathrm{TN}=2234$ \\
\hline Column Total & $\mathrm{P}=2428$ & $\mathrm{~N}=2428$ \\
\hline \multicolumn{2}{|c|}{ Performance metrics for grey scale features } \\
\hline TP rate $=64.209226 \%$ & TN rate $=92.009885 \%$ & \\
\hline FP rate $=7.990115 \%$ & FN rate $=35.790774 \%$ & \\
\hline
\end{tabular}


With the distorted face images added into our data set, it shows a degrading of classification performance. Due to a similarity between distorted face images and distorted non-face images, it makes it difficult for our system to separate the two patterns. It leads to a drop in TP rates to $64 \%$, and TN rates of $92 \%$ and an increase in FP rates to $7.99 \%$ and FN rate to $35.79 \%$. In this data set, it shows a limitation of our system with grey scale features in separating face and non-face images.

\subsubsection{The advantages and disadvantages of texture correlation features}

\subsubsection{Data set 1}

In data set 1, our system with texture correlation features performs "too" well (see figure 4.6). In fact, our system does not have any problem at scramble level 22. The reason why texture correlation features perform so well is the fact that scrambling pixels of an image leads to a major texture change. As texture changes, the amplitude of texture correlation will reduce dramatically. This provides a means for separating the two classes (Texture versus non-texture). However, it does not mean that the texture correlation feature is an excellent feature. If we examine figure 4.6, we see that even at scramble level 7 , texture correlation is able to separate the 2 classes. The problem is that at level 7 , nonface images are not distorted yet. Our system with texture correlation features will have difficulty separating slightly distorted face images (images scrambled at level 7 to 20) from completely distorted face images (images at scramble level 22 and above). This might lead to a negative impact on system performance in real world application. We must take this into consideration with regards to texture correlation limitations.

\subsubsection{Data set 2}

In figure 4.7, our system performs very well at scramble level 10 and above. A ROC curve for data set 2 at scramble level 10 is shown in figure 4.8. However, similar to data set 1 , our system can separate the two classes even at scramble level 5 with a TP rate of $95.79 \%$ 


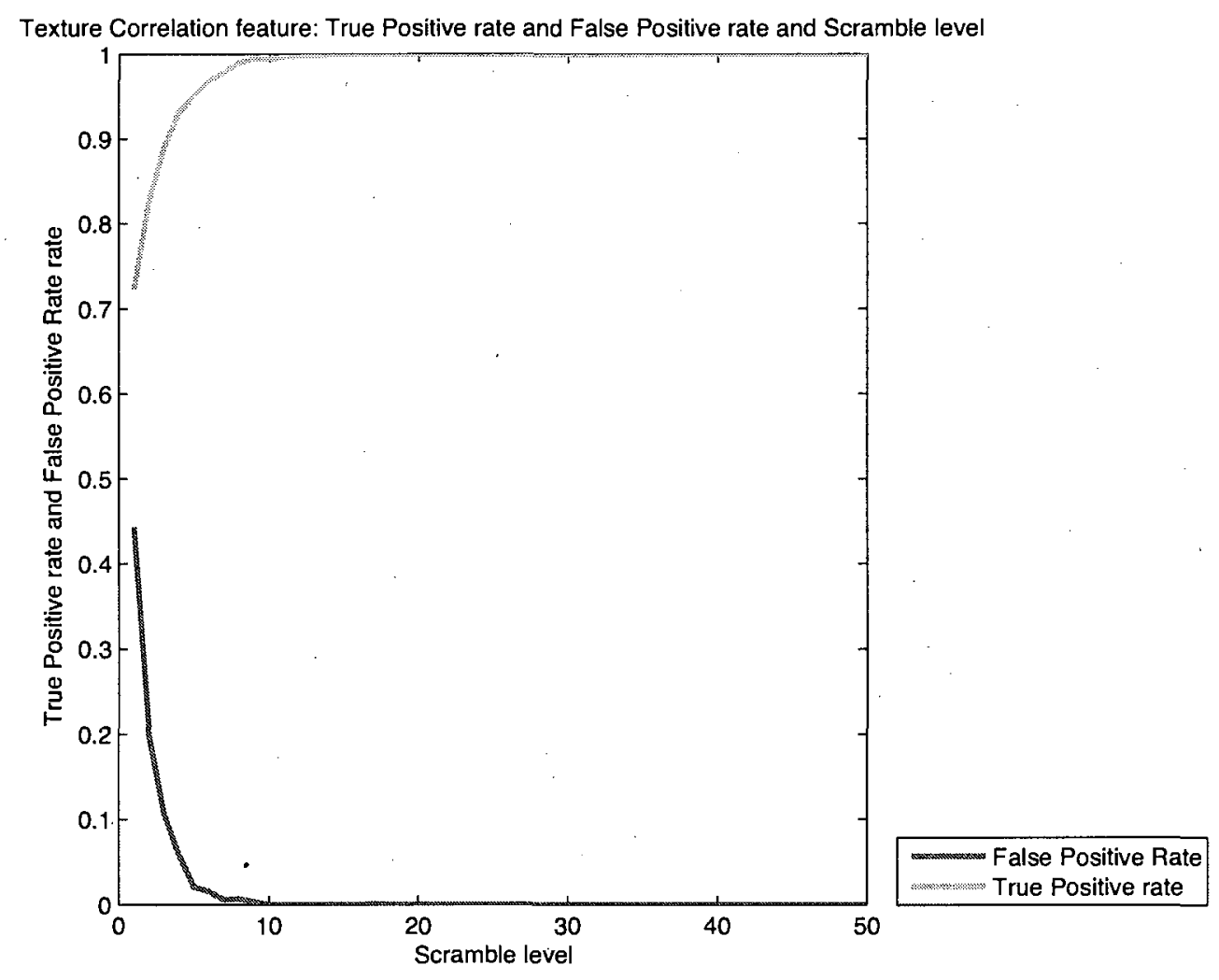

Figure 4.6: Our experimental results of our system with texture correlation features for data set 1

and FP rate of $6.01 \%$. At scramble level 5, non-face images are not completely distorted yet. We will show the problems with texture correlation features in our data set 3 . 


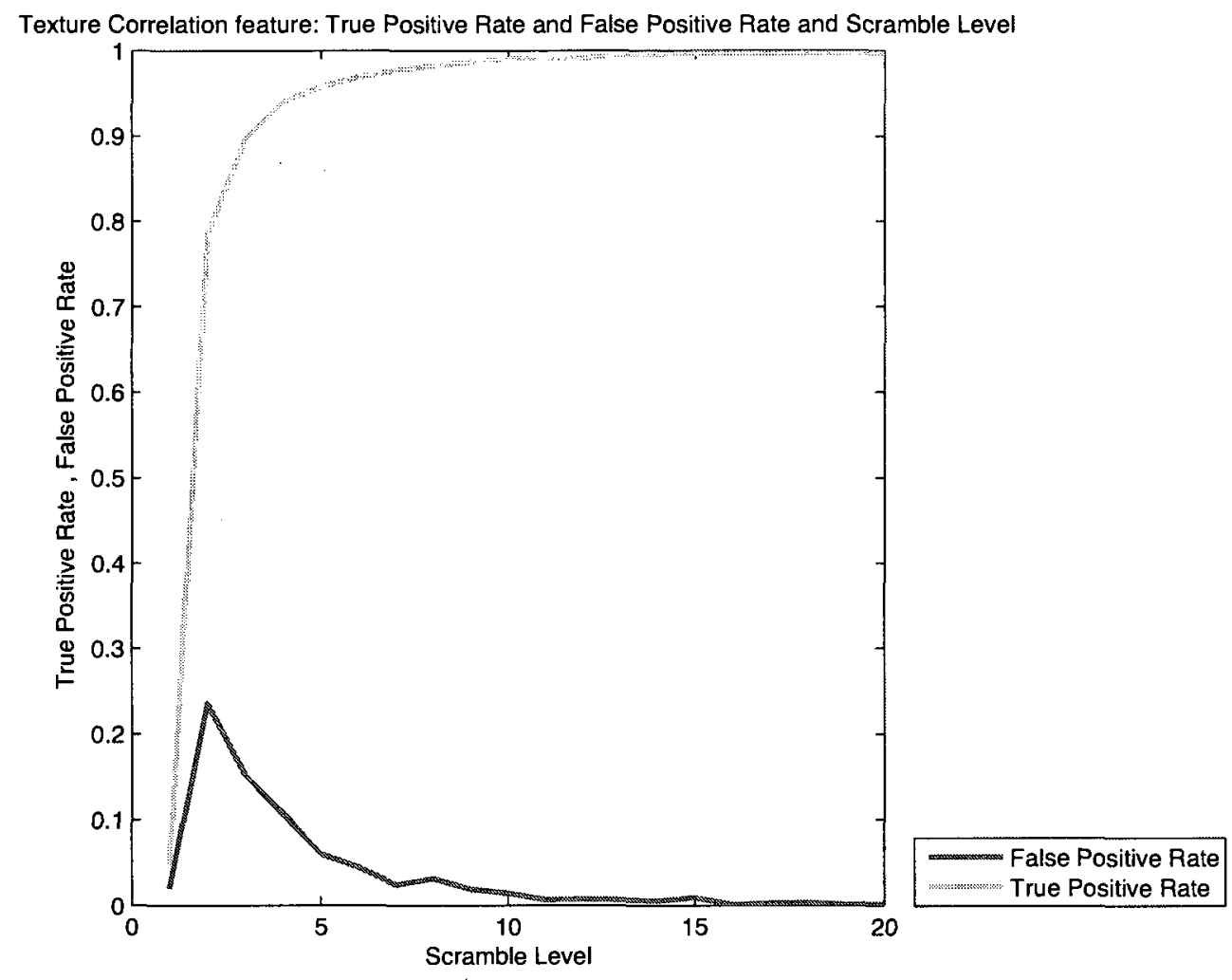

Figure 4.7: Our experimental results of our system with texture correlation features for data set 2

Table 4.4: Confusion Matrix of ROC curve (figure 4.8) and Performance Metrics for our data set 2 at scramble level 10

\begin{tabular}{|l|l|l|}
\hline \multicolumn{3}{|c|}{ Texture correlation features } \\
\hline Hypothesis class & True Positive Class $\mathbf{p}$ & True Negative Class n \\
\hline Positive Class $\mathbf{Y}$ & $\mathrm{TP}=1203$ & $\mathrm{FP}=17$ \\
\hline Negative Class $\mathbf{N}$ & $\mathrm{FN}=12$ & $\mathrm{TN}=2413$ \\
\hline Column Total & $\mathrm{P}=1215$ & $\mathrm{~N}=2430$ \\
\hline \multicolumn{2}{|c|}{ Performance metrics for texture correlation features } \\
\hline TP rate $=99.012346 \%$ & TN rate $=99.300412 \%$ & \\
\hline FP rate $=0.699588 \%$ & FN rate $=0.987654 \%$ & \\
\hline
\end{tabular}




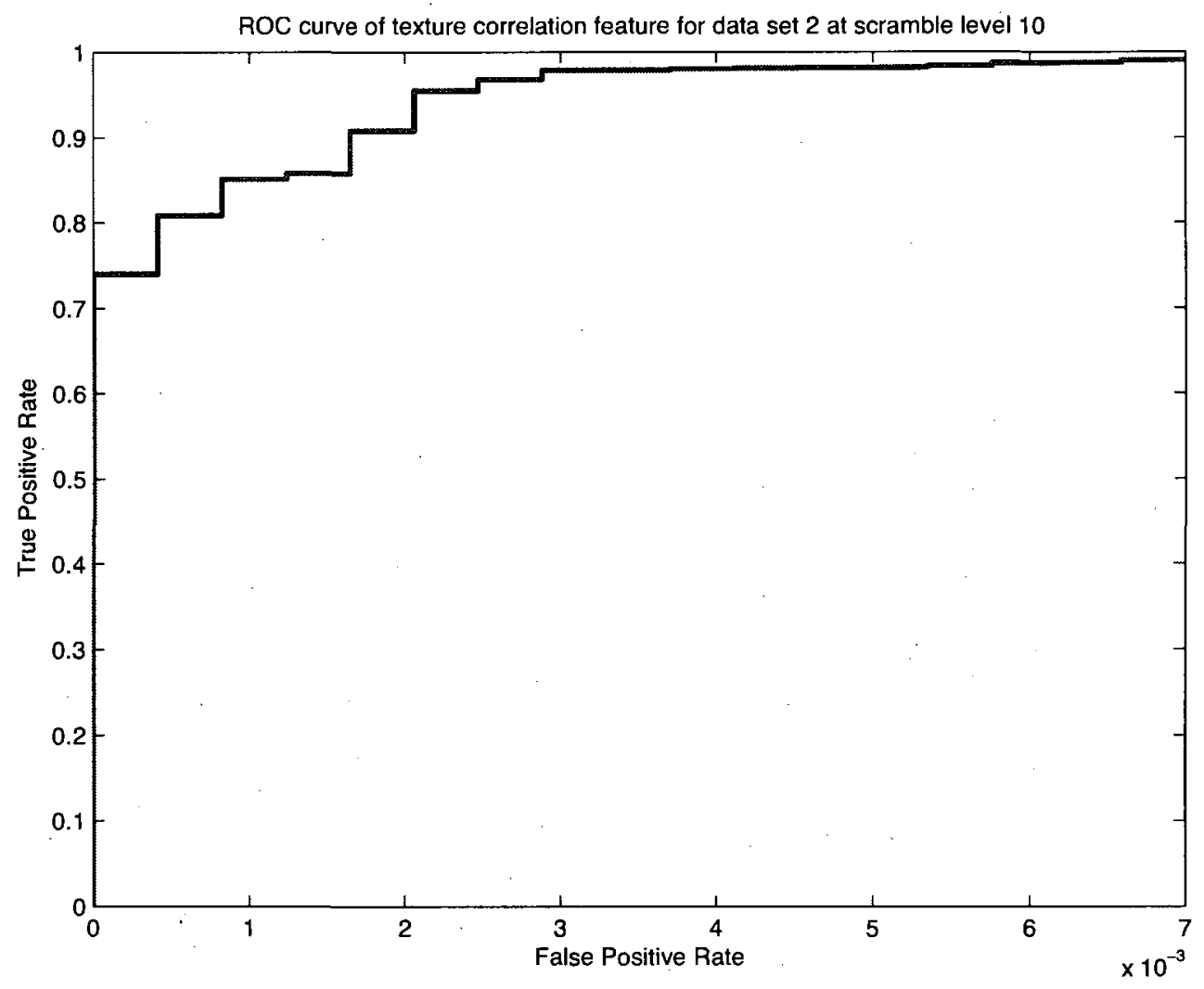

Figure 4.8: ROC curve of texture feature correlation features for our data sets 2 at scramble level 10

\subsubsection{Data set 3}

Similar to our system with grey scale features, we tested our system with texture correlation features using data set 3. As distorted face images are added into our data set, the classification performance of our system with texture correlation features is decreased to a $\mathrm{TP}$ rate of $79 \%$, a $\mathrm{FP}$ rate of $6.5 \%$, a $\mathrm{FN}$ rate of $20.85 \%$ and a $\mathrm{TN}$ rate of $93 \%$ it shows that as face images are slightly distorted, it can create a challenging conditions for our system. It also shows limitations of our texture correlation features. If we compare our system performance on data sets 1 and 3, a drop in classification performance of nearly $20 \%$ is observed. In fact, a real application that contains more distorted face images might have 
a negative impact on our system with texture correlation features. Detailed experimental results for data set 3 are shown in figure 4.9 and table 4.5 .

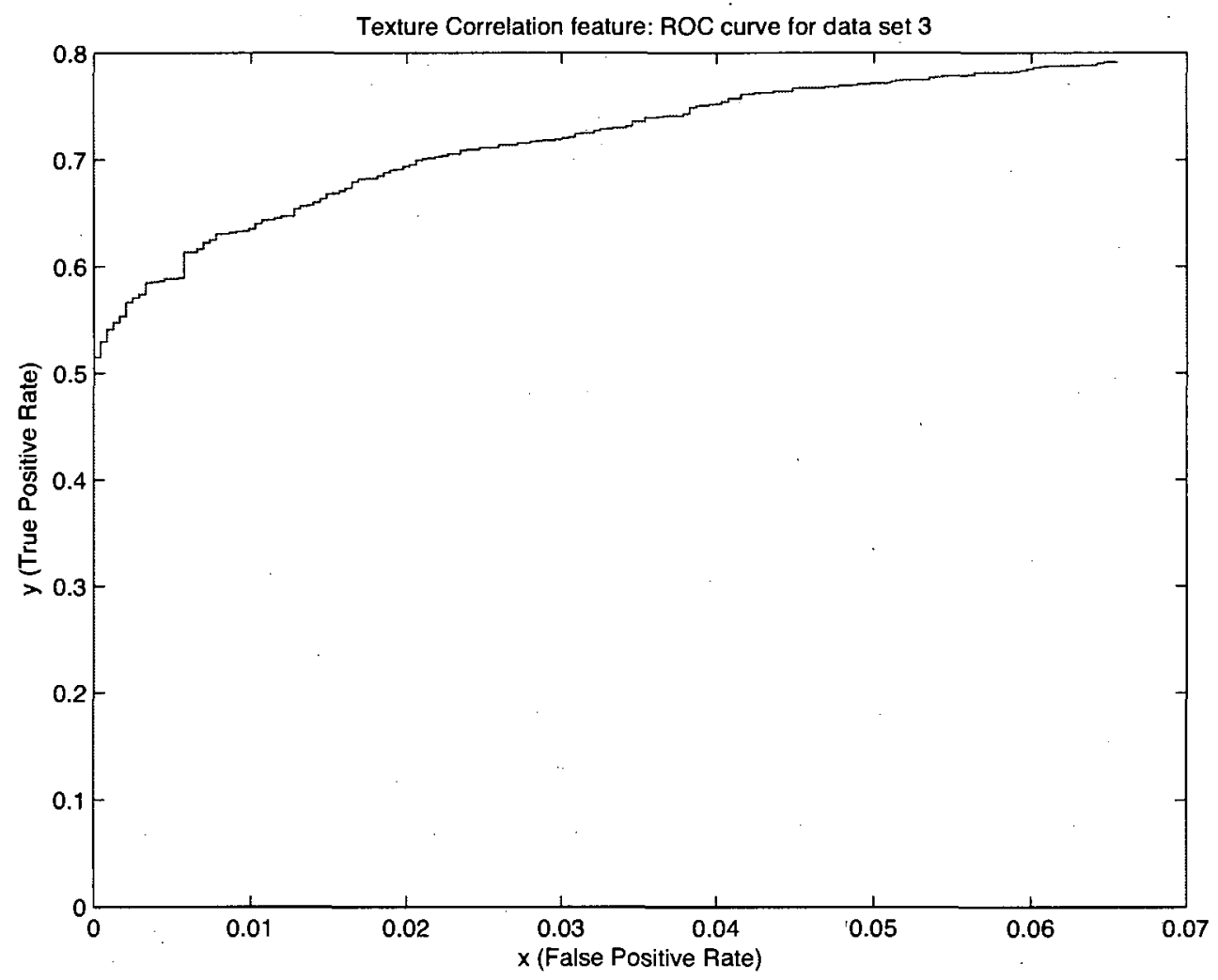

Figure 4.9: ROC curve for our system with texture correlation features for our data set 3 
Table 4.5: Confusion Matrix of ROC curves (figure 4.9) and Performance Metrics for our data set 3

\begin{tabular}{|l|l|l|}
\hline \multicolumn{3}{|c|}{ Texture correlation features } \\
\hline Hypothesis class & True Positive Class $\mathbf{p}$ & True Negative Class $\mathbf{n}$ \\
\hline Positive Class $\mathbf{Y}$ & $\mathrm{TP}=1922$ & $\mathrm{FP}=159$ \\
\hline Negative Class $\mathbf{N}$ & $\mathrm{FN}=506$ & $\mathrm{TN}=2269$ \\
\hline Column Total & $\mathrm{P}=2428$ & $\mathrm{~N}=2428$ \\
\hline \multicolumn{3}{|c|}{ Performance metrics for Texture correlation features } \\
\hline TP rate $=79.159802 \%$ & TN rate $=93.451400 \%$ & \\
\hline FP rate $=6.548600 \%$ & FN rate $=20.840198 \%$ & \\
\hline
\end{tabular}

\subsubsection{Our images}

The purpose of this section is to provide a visualization of the scrambled images in our data. sets. We present a few sets of face images and non-face scrambled images. It is important because it allows us to visually compare the closeness of our non-face images and face images. We will provide some of our images from data sets 1,2 , and 3 . We also provide the non-face images from the MIT data set [20] for comparison purposes. Firstly, we shows face images in our data sets. Secondly, we shows our non-face scrambled images. Our face images are taken from the MIT data set [20]. Figure 4.14 shows the visual definition of face images in our data set. 


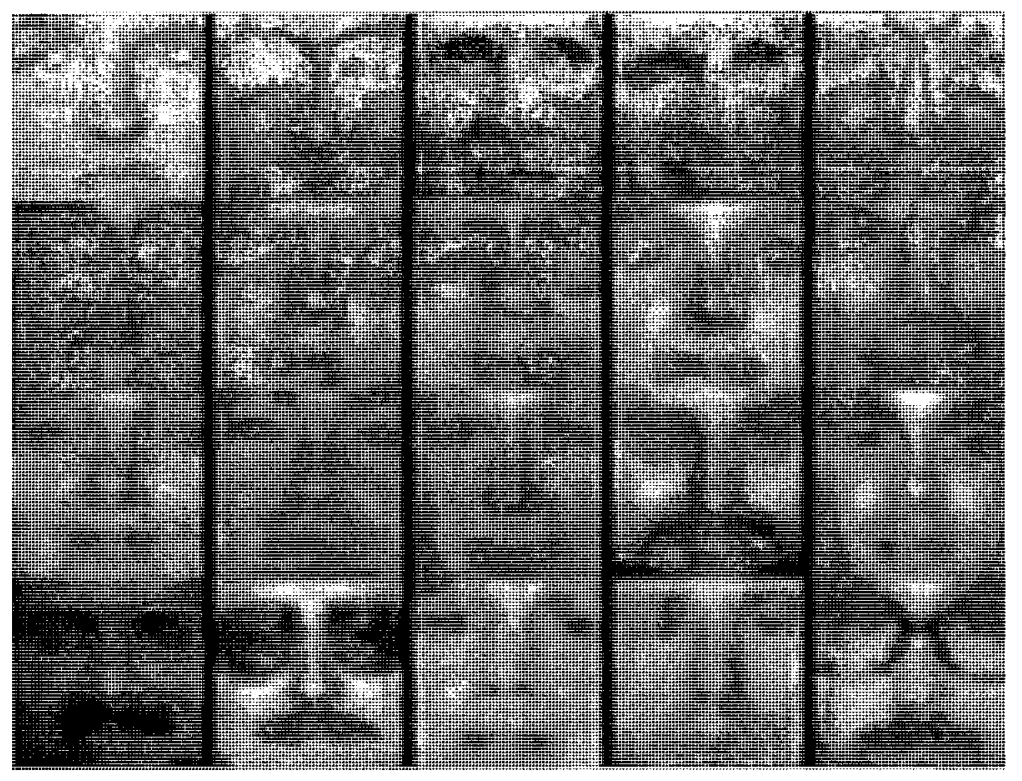

Figure 4.10: Face images are from training set from [20]

In data set 1 , our non-face images are obtained by scrambling face images by pixel from level 1 to level 50. At level 22, most face features are distorted; non-face images are shown in figure 4.11. At scramble levels 25 to 45 , face images are completely distorted. Figure 4.12 shows non-face images scrambled at levels 25 to 45 .

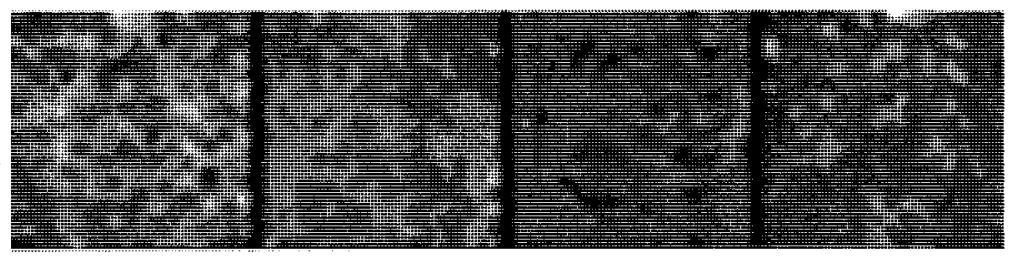

Figure 4.11: Non-face images of data set 1 are generated from face images that are scrambled at level 22 


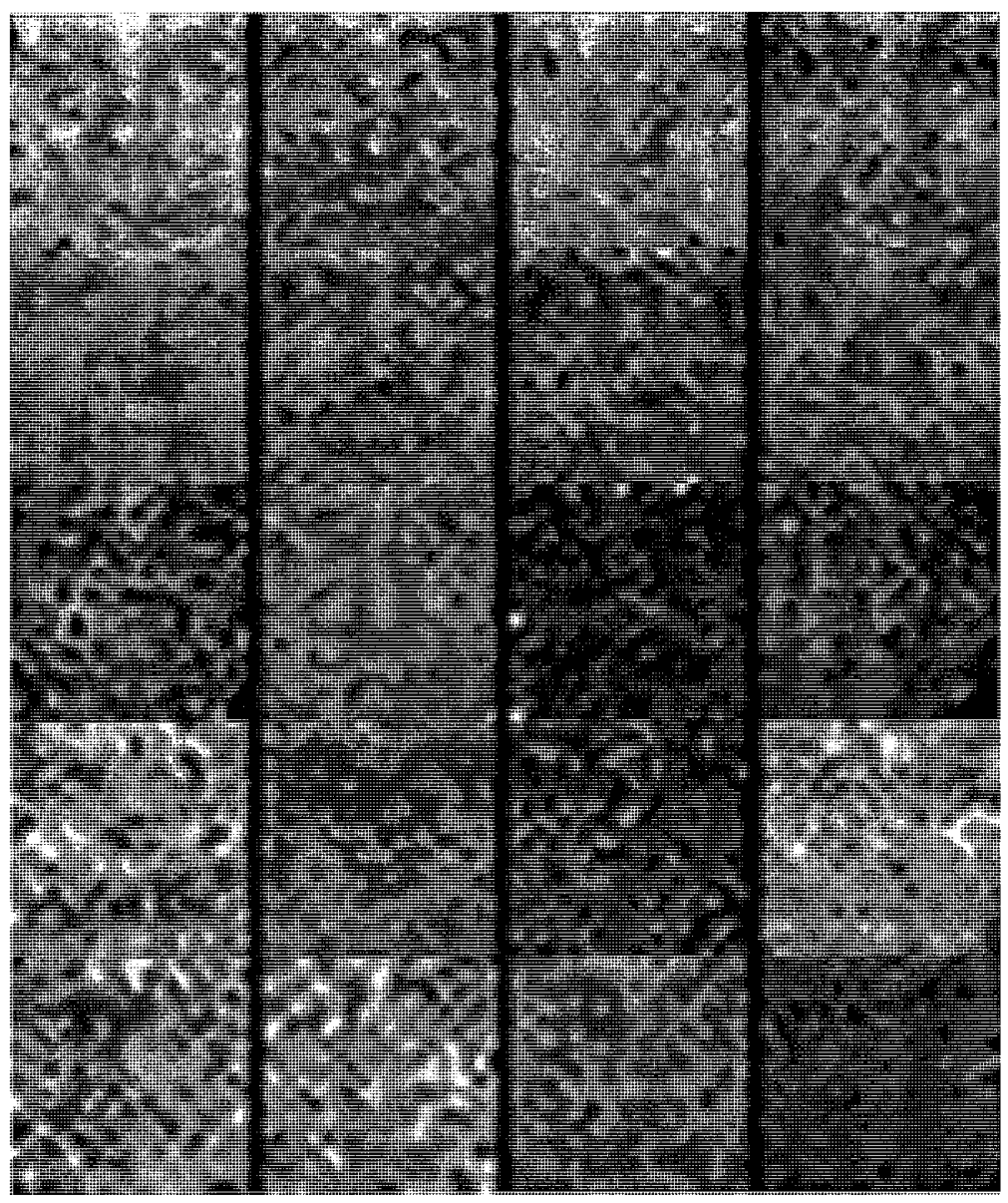

Figure 4.12: Each row of non-face images are generated from face images where pixels are randomly scrambled at levels $25,30,35,40$, and 45 respectively

In data set 2 , we scramble the rows and columns of face images to obtain non-face images. At scramble level 10, face images are distorted into non-face images. Figure 4.13 and figure 4.14 show our non-face images scrambled at levels 10 to 15 .

In data set 3 , we use data set 1 to create our data set 3 . We add slight distorted face images into our data set as described in chapter 3 . Figure 4.15 shows our slightly distorted images at scramble level 19. For non-face images at scramble level 25 and 28, our non-face images are very similar to images in figure 4.12 . 


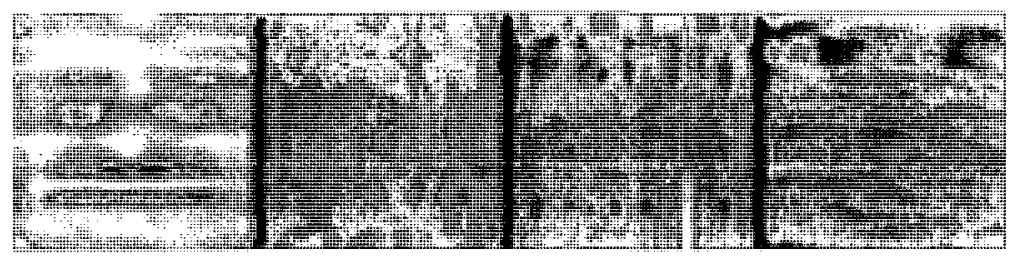

Figure 4.13: Non-face images of data set 2 are generated from face images where columns and rows are randomly scrambled at level 10

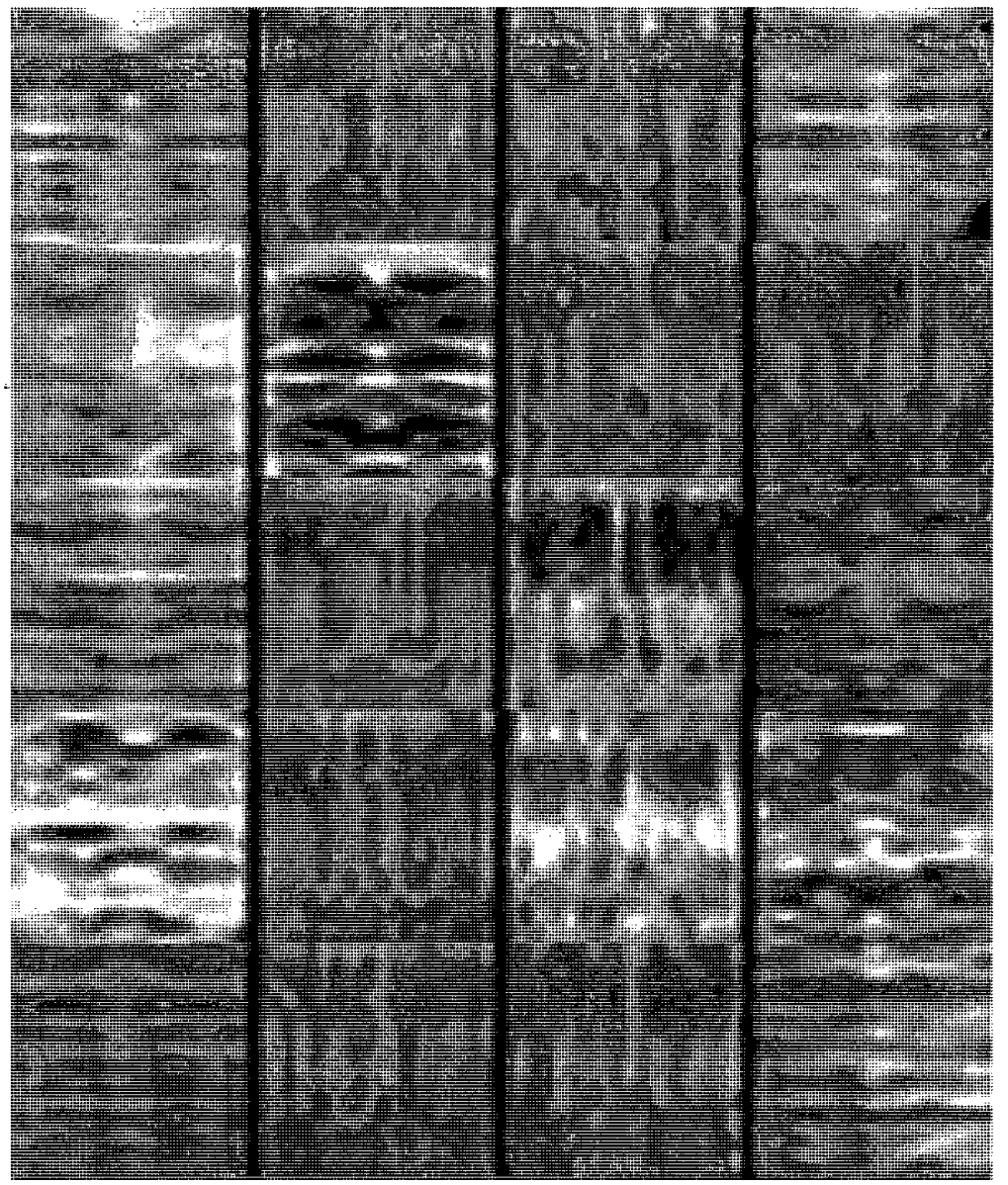

Figure 4.14: Each row of non-face images are generated from face images where columns and rows are randomly scrambled at levels $11,12,13,14$, and 15 respectively 


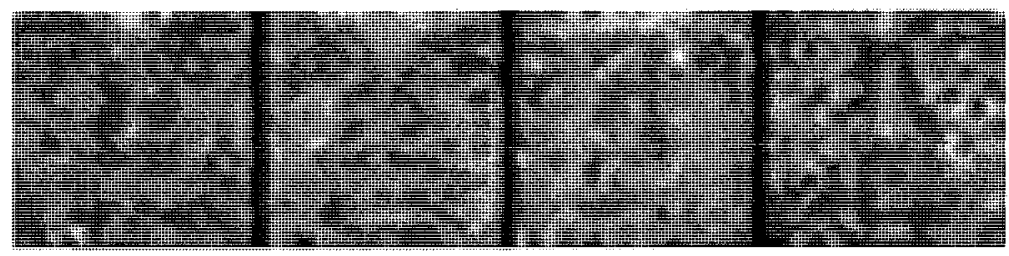

Figure 4.15: Data set 3 face images generated from face images at scramble level 19

For comparison purpose, we provide the non-face images from the MIT data set [20]. Both figure 4.16 and figure 4.17 show that there is a great difference between face and non-face images. For this reason, our perceptron performs well on the MIT data set.

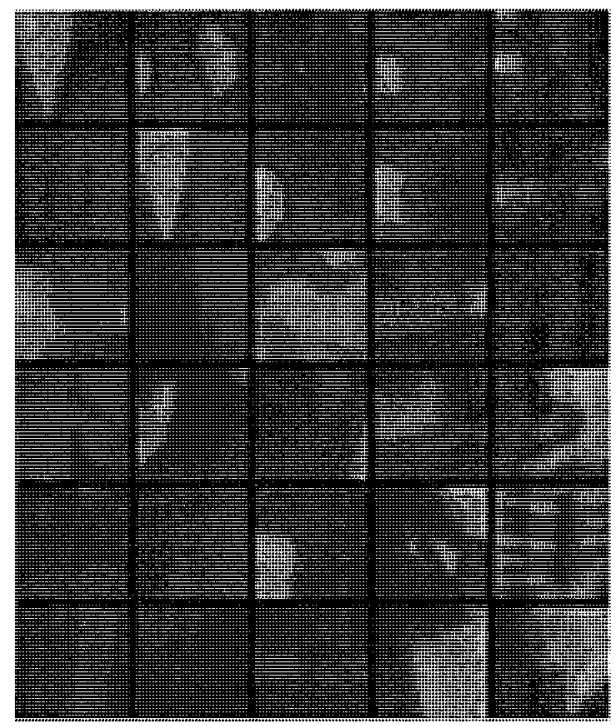

Figure 4.16: Non-face images from the MIT data set [20]

\subsubsection{Comparison between grey scale and texture features}

The above sections provided the experimental results of our system with texture correlation and grey scale features. Our experiments show that each feature has advantages and disadvantages. To put each feature into a real life application, these strengths and weaknesses 


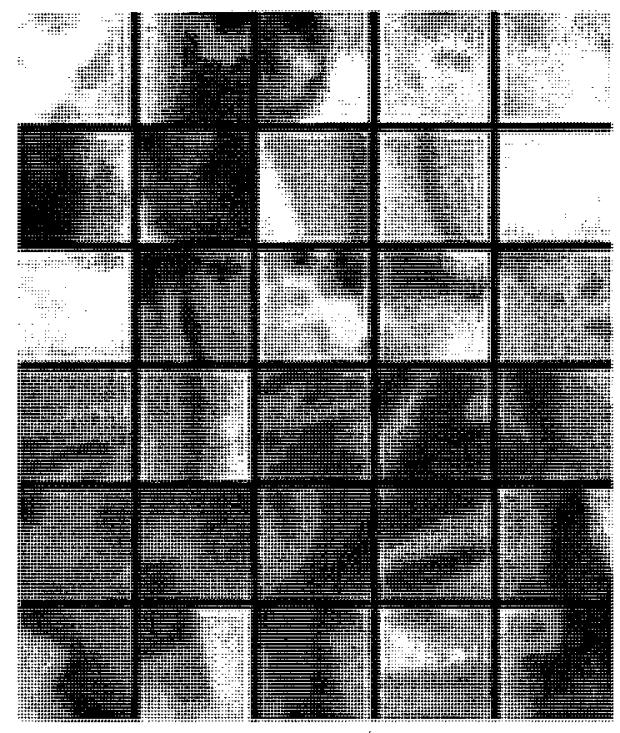

Figure 4.17: Non-face images are from MIT data set [20]

must be acknowledged. In this section, we will provide a comparison of our system performance between systems with grey scale and texture correlation features. We recapture our experimental results and present the combined figures of our system with grey scale and texture correlation features. 


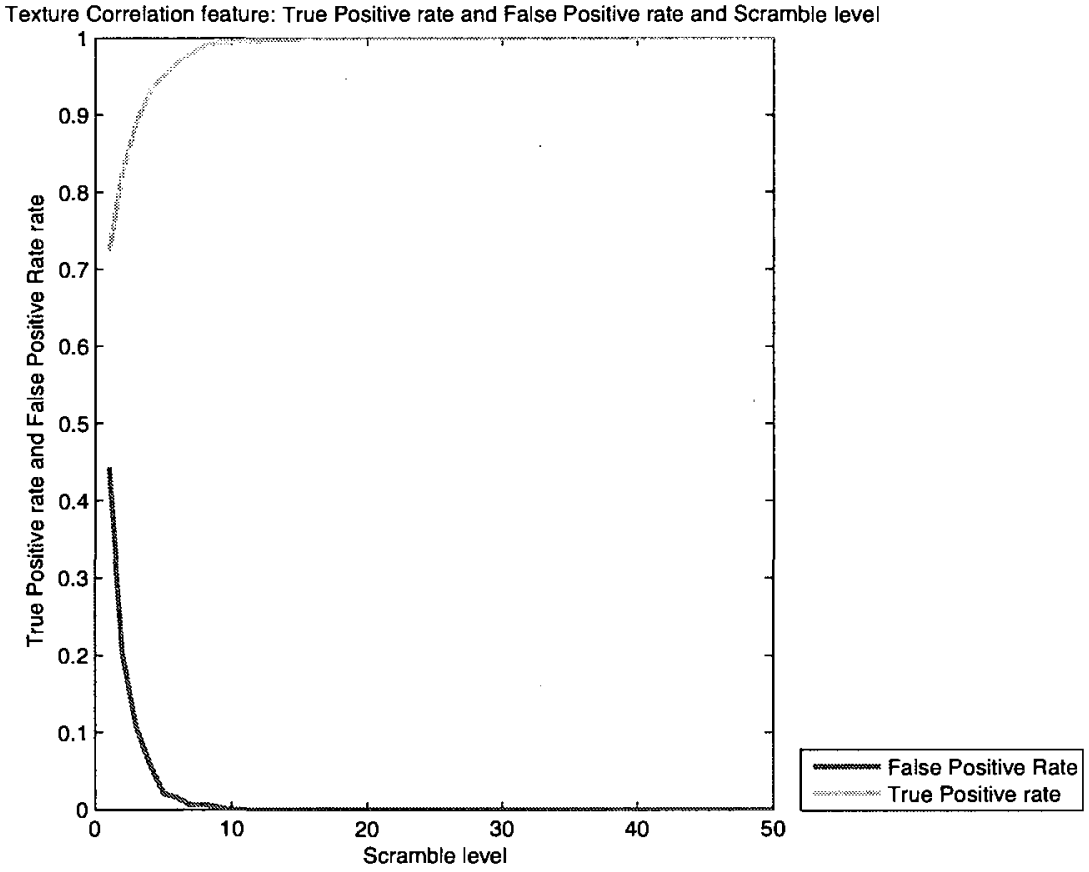

Grey Scale feature: True Positive rate and False Positive rate and Scramble leve

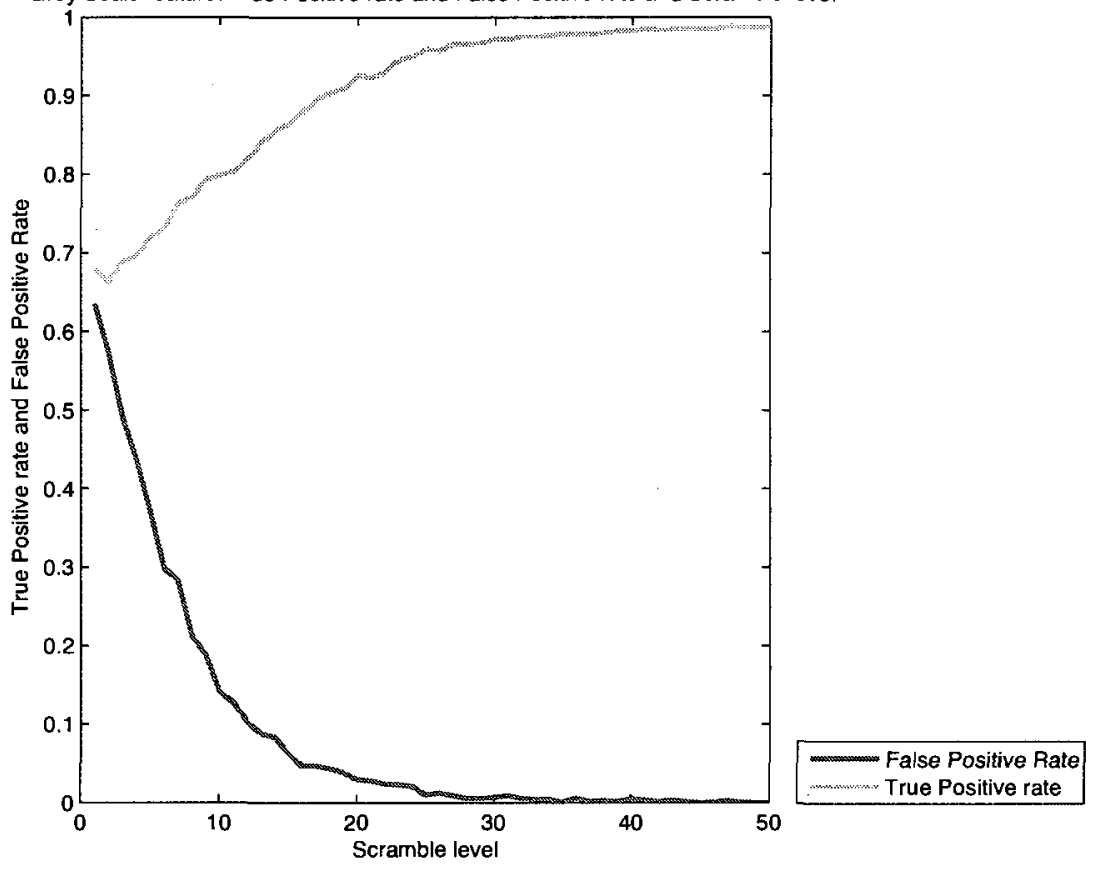

Figure 4.18: Our experimental results of our system with texture correlation and grey scale features for data set 1 


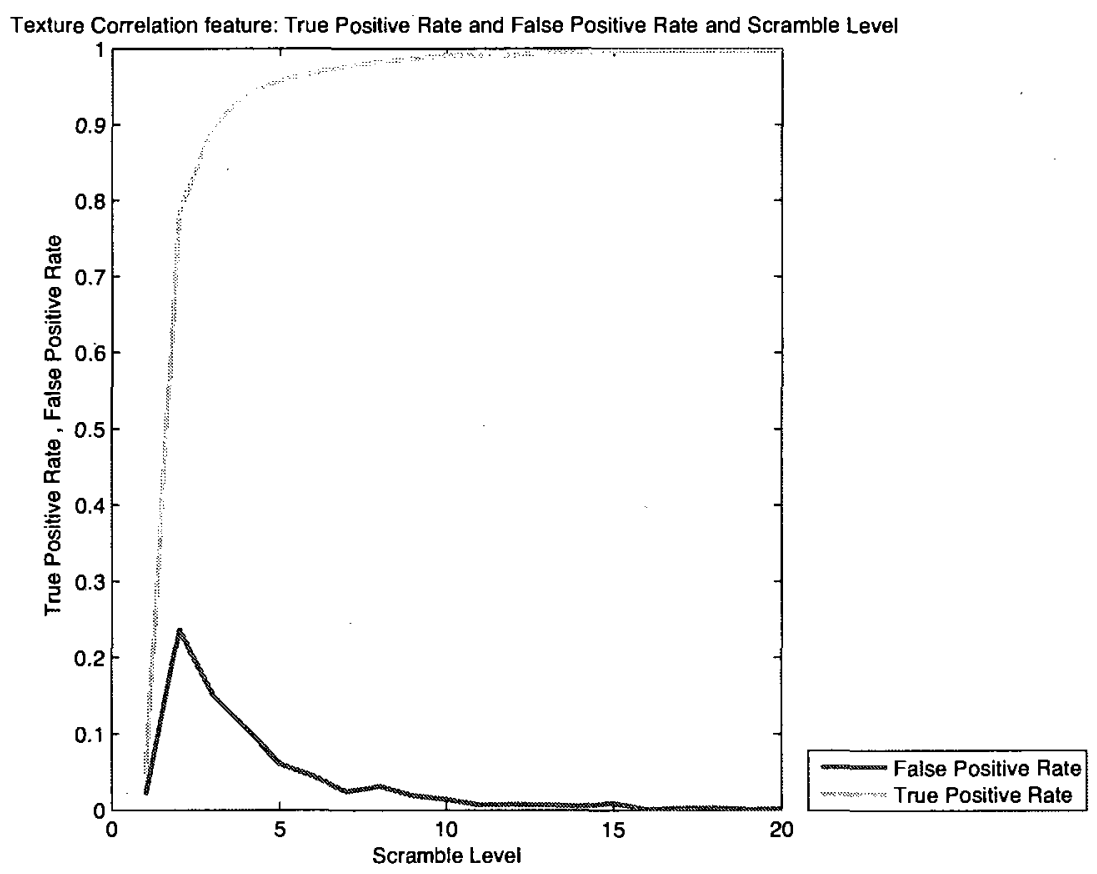

Grey Scale feature: True Positive Rate and False Positive Rate and Scramble Leve

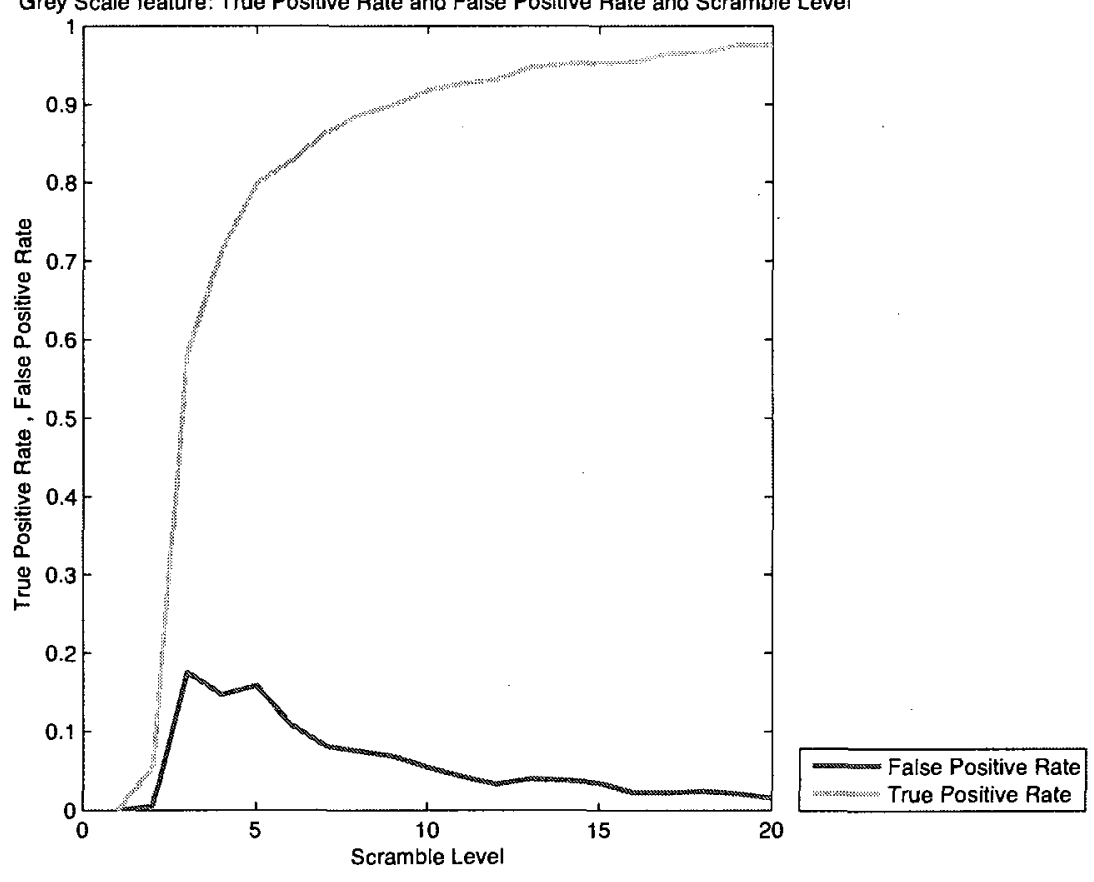

Figure 4.19: Our experimental results of our system with texture correlation and grey scale features for data set 2 

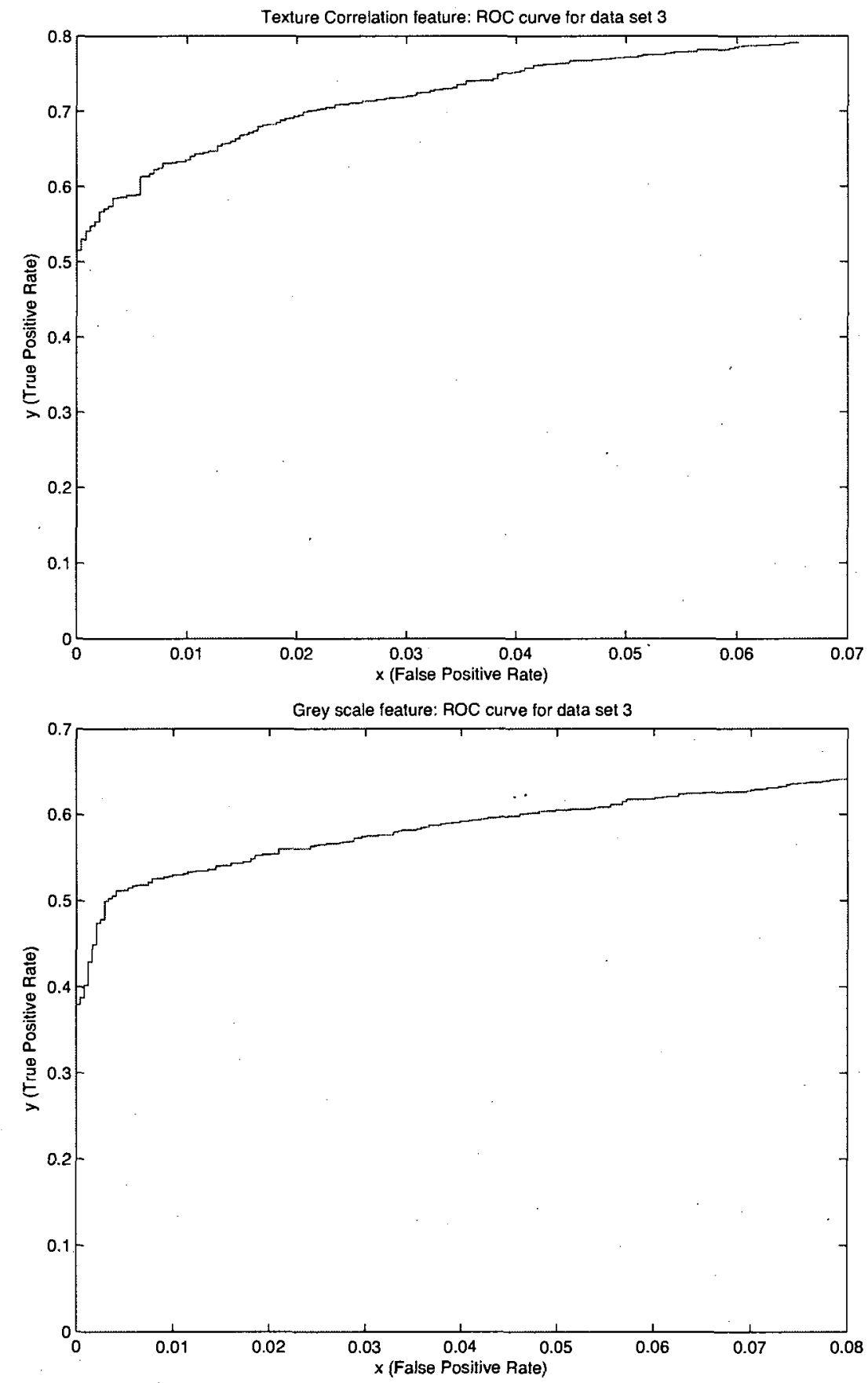

Figure 4.20: Our experimental results of our system with texture correlation and grey scale features for data set 3 
Figure 4.18 , figure 4.19 , and figure 4.20 show the overall performance of our system. The grey scale system has a good performance at scramble level 30 and above for data set 1 and at scramble level 19 and above for data set 2 . It has some problems at scramble level 22 for data set 1 and at scramble level 10 to 18 for data set 2 and with data set 3 . Unlike grey scale features, the texture correlation system performs well at scramble level 22 and above for data set 1 and scramble level 10 and above for data set 2. However, with our data set 3 , both of our system with grey scale and texture correlation features do not work so well as shown in figure 4.5 and figure 4.9. Our system. with texture correlation and grey scale features has a problem in dealing with slightly distorted face images. These cases might lead to a negative impact on system performance. Our two systems have the same structure. The only difference in complexity is the extraction cost of the feature. Texture correlation feature extraction time is slower than grey scale due to the calculation of GLCM matrices, and the statistic mean and deviation of GLCM matrices. Thus the right quantization level will be important for the application of texture correlation.

\subsection{Quantization level and its extraction time}

Quantization level and its extraction time are important factors for texture feature applicability. In this section, we present our findings on quantization level and its extraction time on our data set 2 scrambled at level 10. Our results show that the quantization level performs very well at quantization level 16 for our data sets as compared to other quantization levels while it has a relatively good extraction time. Our results also show that quantization level might affect classification performance if chosen incorrectly. Therefore a good quantization level should be chosen to have a fast extraction time and relatively good classification performance. The results of our experiments for various quantization levels and extraction times are shown in in figure 4.21 and figure 4.22. 


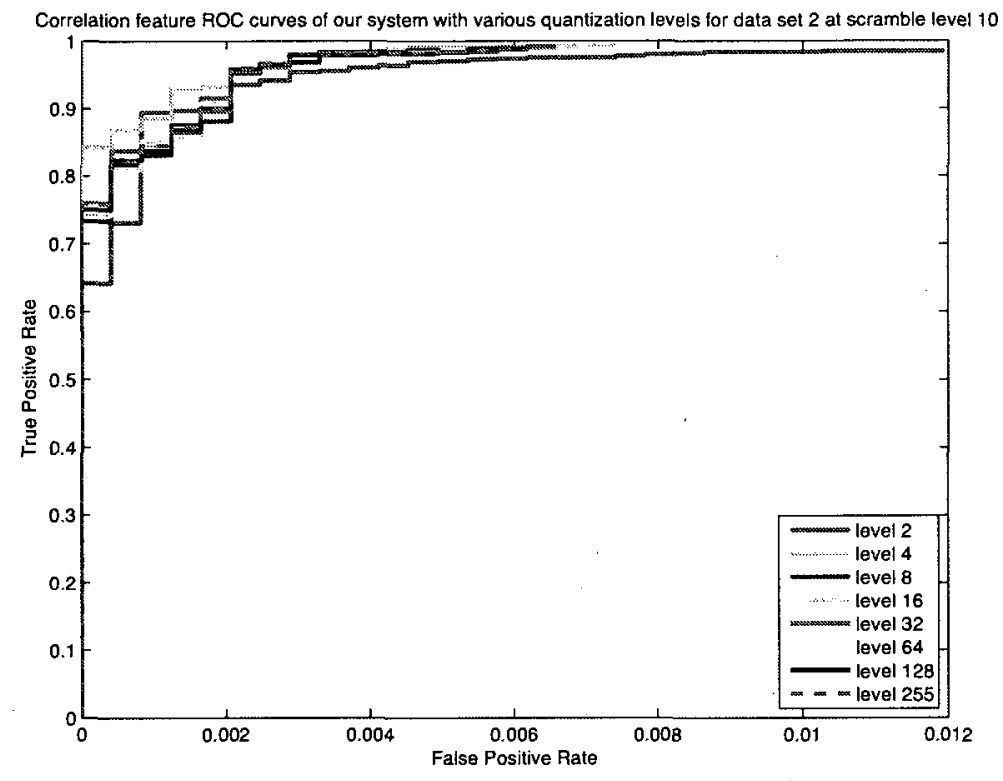

Figure 4.21: ROC curves of various quantization levels

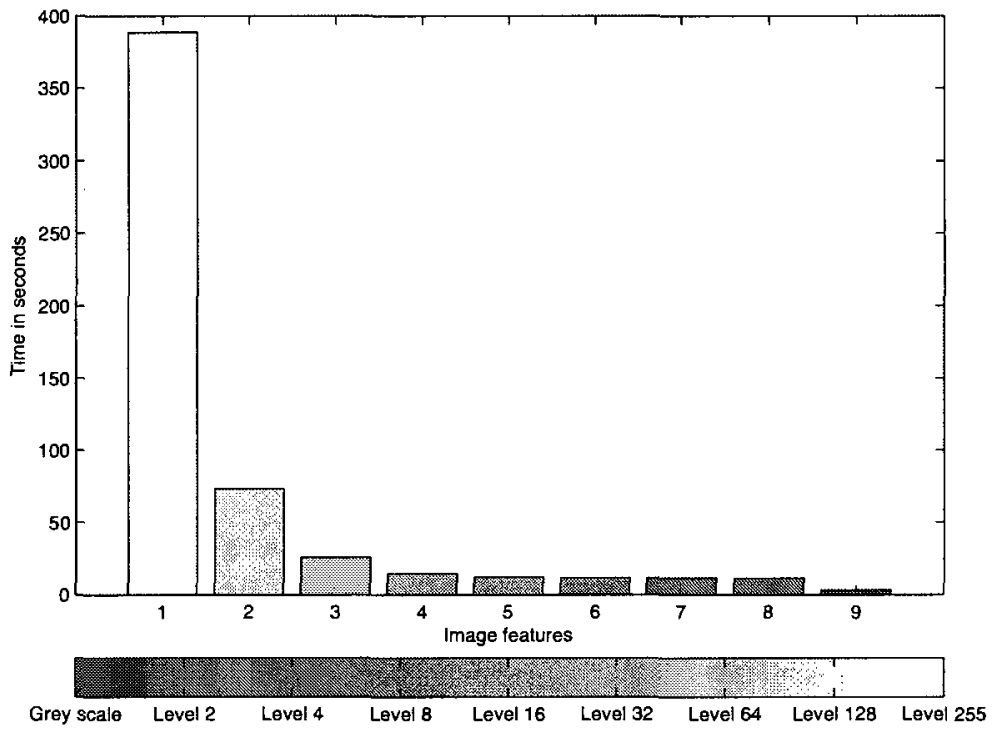

Figure 4.22: Extraction times of correlation texture feature with various quantization levels and grey scale feature for 288 images 


\subsection{Conclusion}

Our experiments allow us to have a better understanding of our system with grey scale and texture correlation features. It shows that neither grey scale nor texture correlation features are a perfect feature. Each has its strengths and weaknesses. The experimental results of our system show that grey scale features have some shortcomings in dealing with non-face patterns that are similar to those in our data sets. Texture correlation features can be used to deal with these cases, however the texture correlation system shows its limitations in our data set 3. These limitations must be taken into consideration. Moreover, quantization level and its extraction time should be carefully chosen to have good classification performance and fast extraction time. All in all, it is a matter of what problem domain we are dealing with. For example, if our problem domain is similar to our data set 1 scrambled at level 22, our texture feature might be a better choice. If our problem domain is similar to the MIT data set, then grey scale might be a better choice. In fact, a realistic face detection system might use multiple features to solve a face detection problem. 


\section{Chapter 5}

\section{Conclusions and Future Work}

\subsection{Conclusions}

Many face detection systems have been proposed to solve very complex face detection problems by generating an extremely large training set. Having an extreme large training set does not mean that you will be able to solve a face detection problem because face image distribution and non-face image distribution are unknown. In order to improve face detection systems, we need to be able to realize the current problems in face detection systems and to ask the "right" questions. In this thesis, we propose a face detection system that uses texture correlation features and SVM to solve a problem of grey scale feature that is not very well addressed. By studying grey scale features and texture features for our face detection system, it allows us to understand their strengths and limitations. Therefore, it allows us to apply them effectively. Our experimental results show that both grey scale image features and texture correlation image features have their strengths and limitations. We can summarize our finding about grey scale and texture correlation systems as follows:

\section{Our system with grey scale features}

- Advantages 
- It works well in cases where face images and non-face images have large differences.

- It has a feature extraction time that is much faster than texture correlation features.

- Disadvantages

- It does not work well with cases in which non-face images are similar to our scrambled non-face images.

- It has large dimensionality that might inhibit it scaling to work with large images.

\section{Our system with texture correlation features}

- Advantages

- It works well with our data sets 1 and 2, i.e. face images and non-face are similar.

- It also works with cases in which face images and non-face images that have large differences.

- It has small dimensionality.

- Disadvantages

- It does not work so well with data set 3 .

- It has a longer extraction time as compared to grey scale features.

- It might have problems with face images that are only slightly distorted.

- Its quantization level must be chosen correctly to obtain a good classification performance and a reasonable extraction time.

Though texture correlation features seem to work well with our data sets 1 and 2, situations that are similar to our data set 3 must be taken into consideration. Quantization levels should be chosen carefully so that classification performance will not be affected by the cost of extraction time. More testing should be done to learn more about the strengths 
and weaknesses of texture correlation features as this will be essential and vital for texture correlation applications.

In this thesis, we also provided a method of generating non-face images. This method can help in generating non-face images and take into account cases where non-face images are more challenging than those in the MIT data set. It provides the definition of non-face patterns. This definition is novel and very important because it defines the severity of the problem to be solved. It is significant because it allows us to test and reconstruct our work. Moreover, these non-face images will facilitate the system in dealing with non-face patterns that are closer to face images. Thus, it will improve the overall performance of the system.

We also proposed a standard evaluation protocol and the use of ROC curve as an evaluation method. They provide a clear and effective way to evaluate system performance as compared to previous evaluation methods and performance metrics. In our experiments, we examine a specific case for our face detection problem. Our experimental results show that it really depends on the definitions of face and non-face image or problem domain that we want to solve using grey scale or texture correlation features. For example, the grey scale system can work with cases where face and non-face images have large differences as in the case of the MIT data sets. However, it will have problems with data set 2 at scramble level 10 and data set 3 with added slightly distorted face images. For texture correlation system, it works well with our data sets 1 and 2 . It has some problems with our data set 3 . These instances shows both limitations of the grey scale system and the texture correlation system. In reality, it will require both features or more features to solve some particular problem domains. It can be cases that combine the MIT data sets and our data set 3 or some other data sets that challenge both of our feature sets. Thus the need to investigate the limitations of a system should always be encouraged and is paramount to the improvement in the face detection field. 


\section{$5.2 \quad$ Future work}

Our goal for future work is to improve appearance-based face detection systems and texture feature applicability in face detection. It is important to realize the essence of having good data sets. Therefore, the construction of good data sets should be encouraged and rewarded. The improvements of SVM or neural network methods should be done after the construction of new challenging data sets in which the problem domain is clearly defined. For texture features, many more studies should be done to learn about the texture properties and its limitation so that texture features can be applied properly in face detection methods. One of the interesting properties of texture correlation is its low dimensionality. It will provide a means to construct appearance-based face detection system for large images which currently is impossible for grey scale features. However, one of the Achilles heels of texture correlation is its extraction time which might hinder its applicability. Therefore, the improvement of extraction speed of texture feature calculation should be studied and addressed. Our method of generating non-face images also has some great implications. Future research should focus on how to generate a meaningful non-face patterns or negative patterns. A good negative sample generating method can eliminate the need to collect or create negative samples which is a very time consuming task. It can lead to better system performance.

The field of appearance-based face detection is still in its infancy. Many improvements are needed to move the field forward. This work has answered some questions related to the use of texture correlation in face detection. It also addresses some short-comings in previous evaluation methods and data sets. It is clear that future work should look at constructing more meaningful standardized data sets and finding the problems that are associated with current face detection systems. With these objectives in mind, we will move the field to another level. 


\section{Bibliography}

[1] M.F. Augusteijn and T.L. Skujca. Identification of human faces through texture-based feature recognition and neural network technology. Proc. IEEE Conf. Neural Networks, pages pp. 392-398, 1993.

[2] F.W. Campbell and J.G. Robson. Application of fourier analysis to the visibility of grat- ings. Journal of Physiology, 197:pp. 551-566, 1968.

[3] J. M Coggins. A Framework for Texture Analysis Based on Spatial Filtering. PhD thesis, Computer Science Department Michigan State University, 1982.

[4] C. Cortes and V. Vapnik. Support vector networks. Machine Learning, vol. 20:pp. $1-25,1995$.

[5] Costas and Pistas. Rule-based face detection in frontal views. Proc. Int'l Conf. Acoustics, Speech and Signal Processing, vol. 4:pp. 2537-2540, 1997.

[6] G.C. Cross and A.K. Jain. Markov random field texture models. IEEE Transactions on Pattern Analysis and Machine Intelligence, PAMI-5:pp. 25-39, 1983.

[7] Y. Dai and Y. Nakano. Face-texture model based on sgld and its application in face detection in a color scene. Pattern Recognition, vol. 29(no. 6):pp.1007-1017, 1996.

[8] J.G. Daugman. Two-dimensional spectral analysis of cortical receptive field profiles. Vision Research, 20:pp. 847-856, 1980. 
[9] H. Derin and H. Elliott. Modeling and segmentation of noisy and textured images using gibbs random fields. IEEE Transactions on Pattern Analysis and Machine Intelligence, PAMI-9:pp. 39-55, 1987.

[10] R. Freund E. Osuna and F. Girosi. Training support vector machines: An application to face detection. Proc. IEEE Conf. Computer Vision and Pattern Recognition, pages pp. 193-199, 1997.

[11] J. P. Egan. Signal Detection Theory and ROC analysis, Series in Cognition and Perception. New York: Academic Press, 1975.

[12] S. Baluja H. Rowley and T. Kanade. Human face detection in visual scenes. Advances in Neural Information Processing System 8, D.S. Touretzky, M.C. Mozer, and M.E. Hasselmo, eds., pages pp. 875--881, 1996.

[13] S. Baluja H. Rowley and T. Kanade. Neural network-based face detection. Proc. IEEE Conf. Computer Vision and Pattern Recognition, pages pp. 203-208, 1996.

[14] S. Baluja H. Rowley and T. Kanade. Neural network-based face detection. IEEE Trans. Pattern Analysis and Machine Intelligence, vol. 20(no. 1):pp. 23-28, January 1996.

[15] J. K Hawkins. Textural properties for pattern recognition.

[16] Simon Haykin. Neural Networks A Comprehensive Foundation. Prentice Hall, 2nd edition, 1999.

[17] S. Y. Kung and J. S. Taur. Decision-based neural networks with signal/image classification applications. IEEE Trans. Neural Networks, vol. 6:pp. 170181, January 1995.

[18] J. Malik and P. Perona. Preattentive texture discrimination with early vision mechanisms. Journal of the Optical Society of America, Series A:pp. 923-932, 1990.

[19] David J. Kriegman Ming-Hsuan Yang and Narendra Ahuja. Detecting faces in images: A survey. IEEE Transaction on Pattern Analysis and Machine Intelligence, January 2002. 
[20] MIT. Cbcl data sets. http://www.ai.mit.edu/projects/cbcl.

[21] M. Propp and A. Samal. The m2vts multimodal face database. Proc. First Int'l Conf. Audio- and Video-based Biometric Person Authentication, 1997.

[22] W. Richards and A. Polit. Texture matching. Kybernetic, 16:pp. 155-162, 1974.

[23] Haralick M. Robert, Shanmugam K., and Dinstein I. Textural features for image classifcation. IEEE Transactions on Systems, Man, and Cybernetics, vol. SMC-3(no. 6):pp. 610-621, November 1973 .

[24] F. Rosenblatt. The perceptron: A probabilistic model for information storage and organization in the brain. Psychological Review, vol. 65(no. 1):pp. 386-408, 1958.

[25] S. Y. Kung S. H. Lin and L. J. Lin. Face recognition / detection by probabilistic decision-based neural network. IEEE Trans. Neural Network, vol. 8(no. 1):pp. 114132, January 1997.

[26] J Sklansky. Image segmentation and feature extraction. IEEE Transactions on Systems,Man, and Cybernetics, SMC-8:pp. 237-247, 1978.

[27] K.A. Spackman. Signal detection theory: Valuable tools for evaluating inductive learning. Proc. Sixth Internat. Workshop On Machine Learning., pages pp.160-163, 1989.

[28] E. Philip Amburn Stephen M. Pizer. Adaptive histogram equalization and its variations. Computer Vision, 39:pp. 355-368, 1987.

[29] K. K. Sung. Learning And Example Selection For Object And Pattern Detection. PhD thesis, Massachusetts Inst. of Technology, 1996.

[30] K. K. Sung and T. Poggio. Example-based learning for view-based human face detection. IEEE Trans. Pattern Analysis and Machine Intelligence, vol. 20(no. 1):pp. 39-52, January 1998.

[31] J. Swets. Measuring the accuracy of diagnostic systems. Science 240, pages pp.12851293, 1988. 
[32] S. Mori Tamura, H. and Y. Yamawaki. Textural features corresponding to visual perception. IEEE Transactions on Systems,Man, and Cybernetics, SMC-8:pp. 460-$473,1978$.

[33] M. Tuceryan and A. K. Jain. Texture segmentation using voronoi polygons. IEEE Transactions on Pattern Analysis and Machine Intelligence, PAMI-12(no. 6):pp. 211$216,1990$.

[34] Cornell University. Svmlight package. http://http://svmlight.joachims.org/.

[35] VN. Vapnik. Statistical Learning Theory. Newyork: Wiley, 1998.

[36] G. Yang and T. S. Huang. Human face detection in complex background. Pattern Recognition, vol. 27(no. 1):pp. 345-350, 1994.

[37] S. W. Zucker and K. Kant. Multiple-level representations for texture discrimination. In Proceedings of the IEEE Conference on Pattern Recognition and Image Processing, SMC-8:pp. 609-614, 1981. Dallas, TX. 
Appendix A

Examples of Face and Non-face

images 


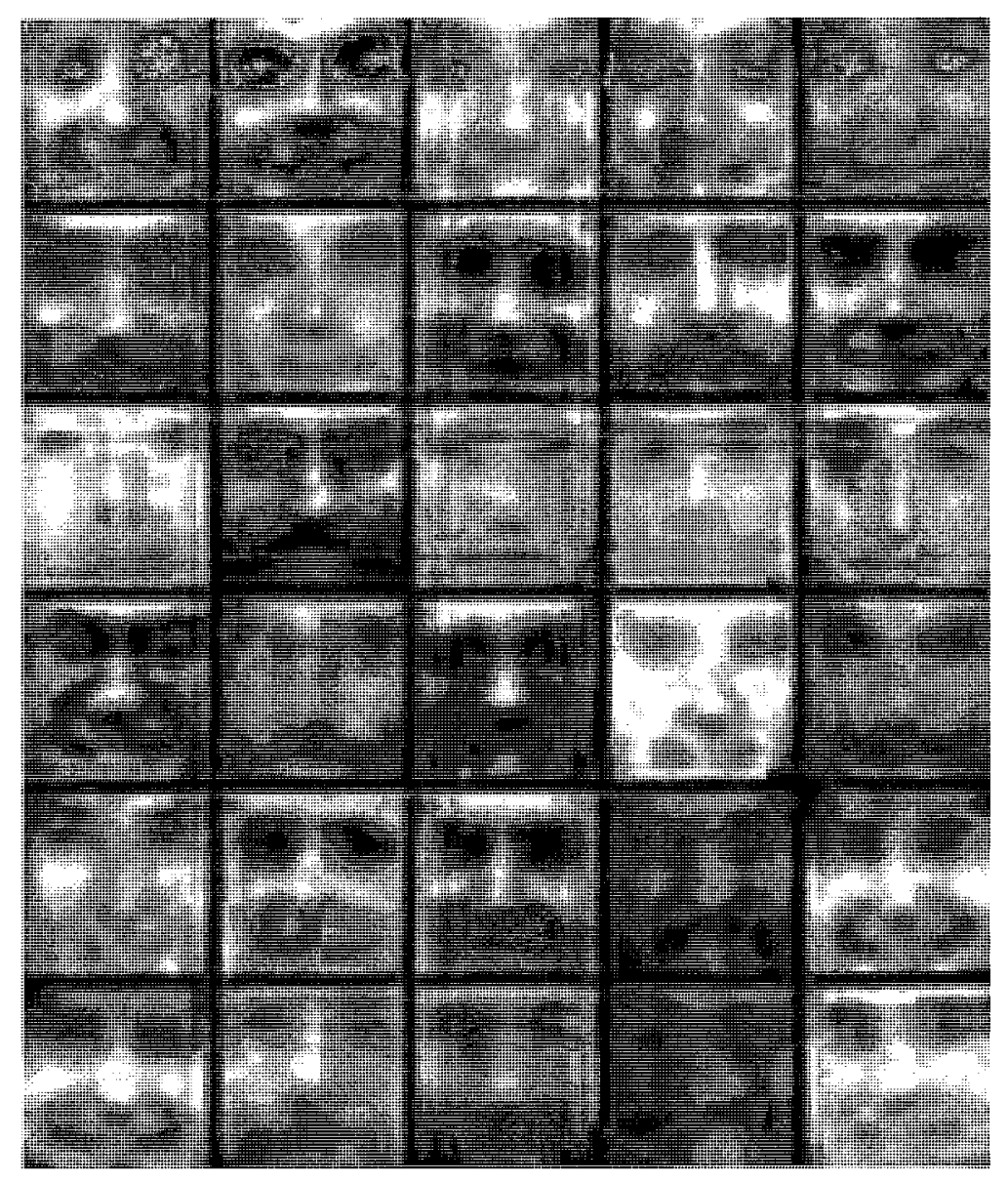

Figure A.1: face images of MIT CBCL training set [20] 


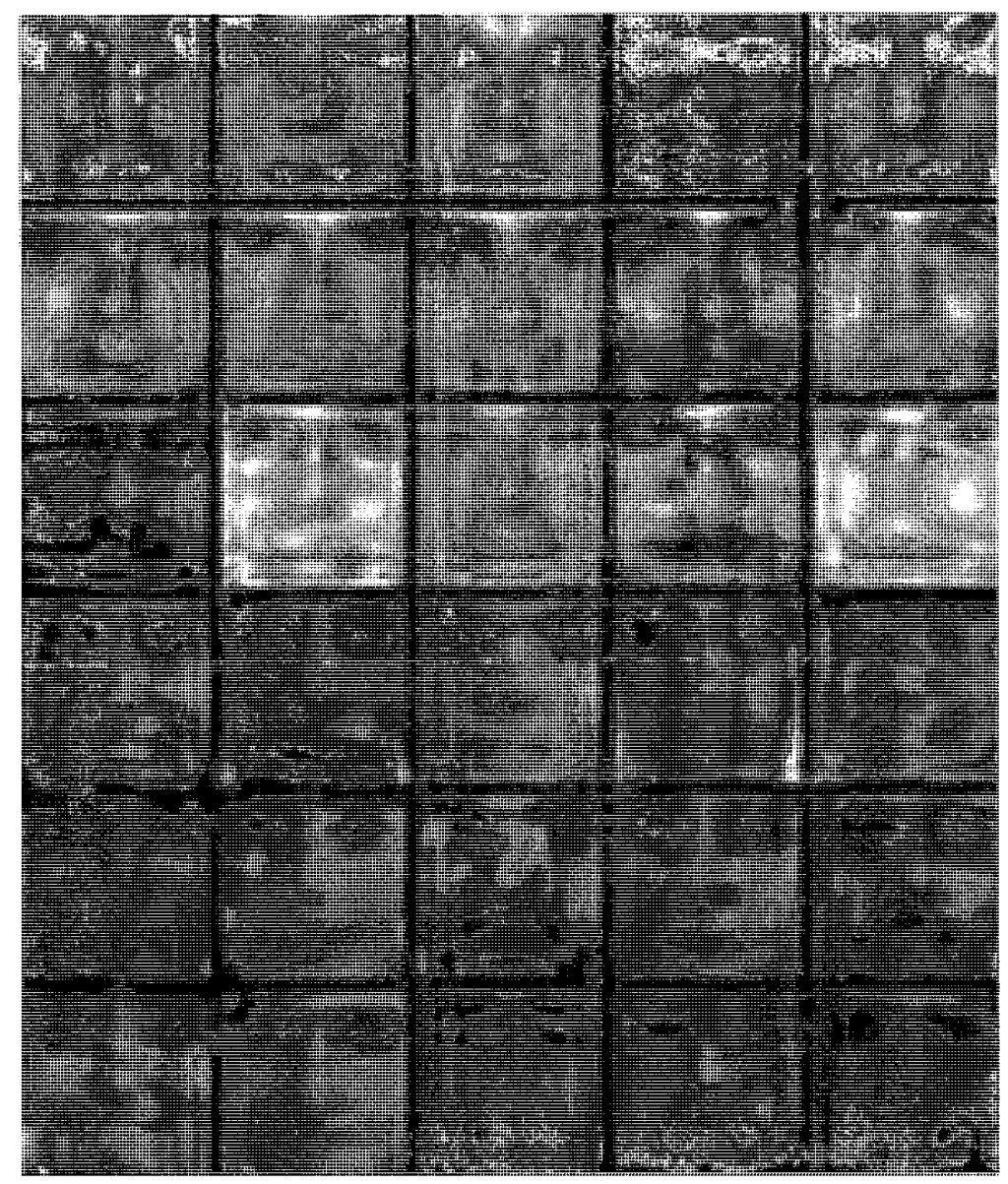

Figure A.2: face images of MIT CBCL training set [20] 


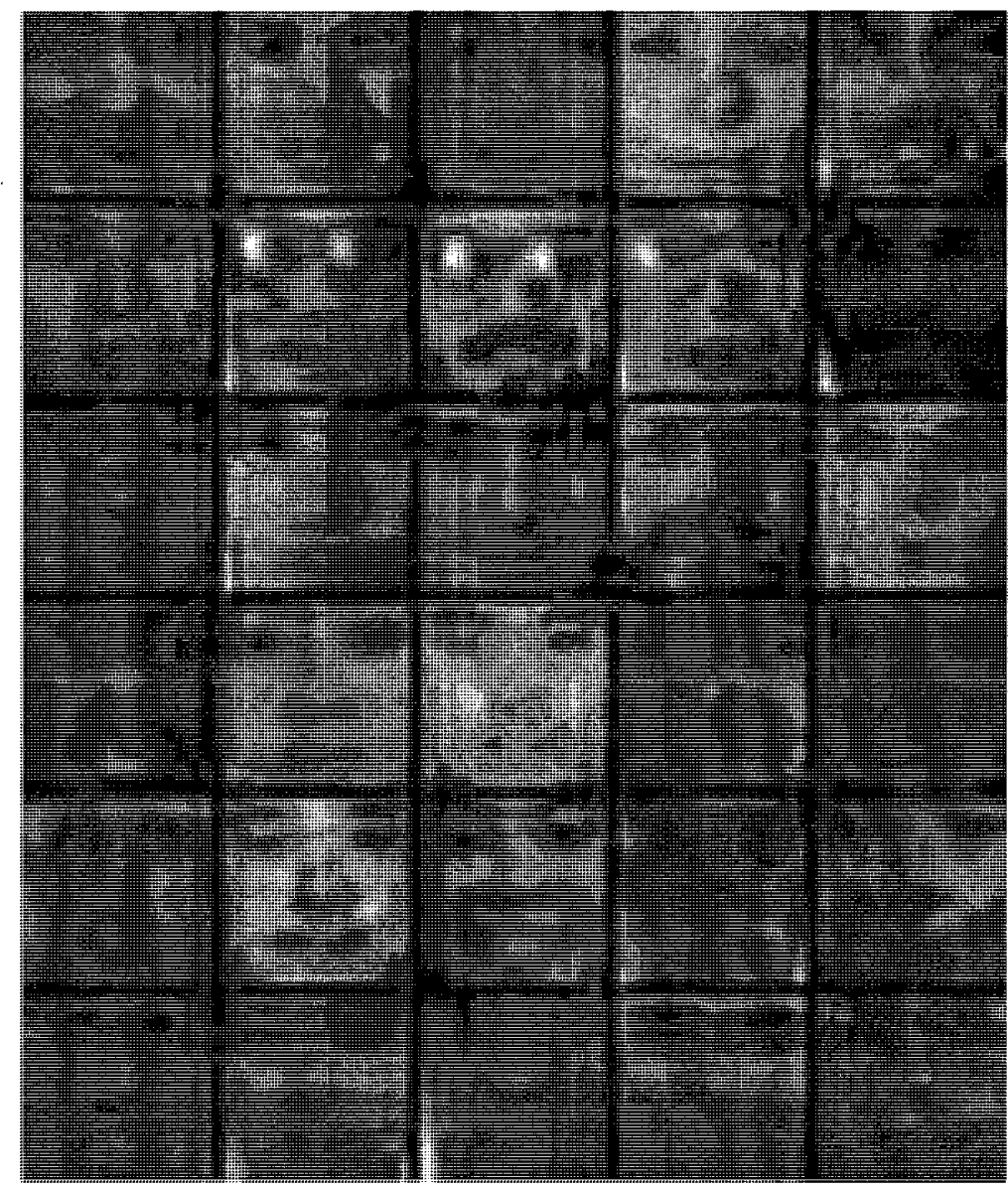

Figure A.3: face images of MIT CBCL training set [20] 


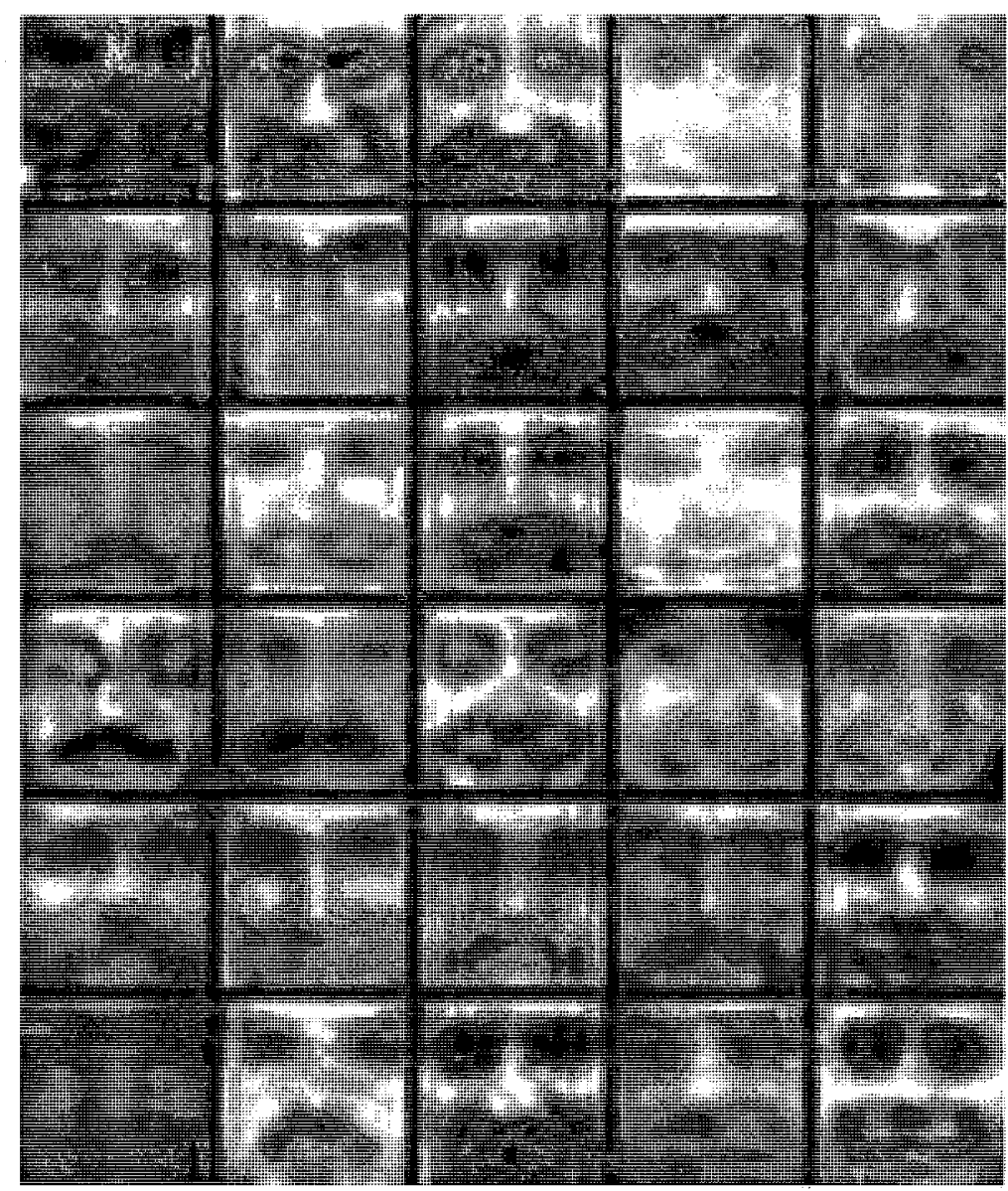

Figure A.4: face images of MIT CBCL training set [20] 


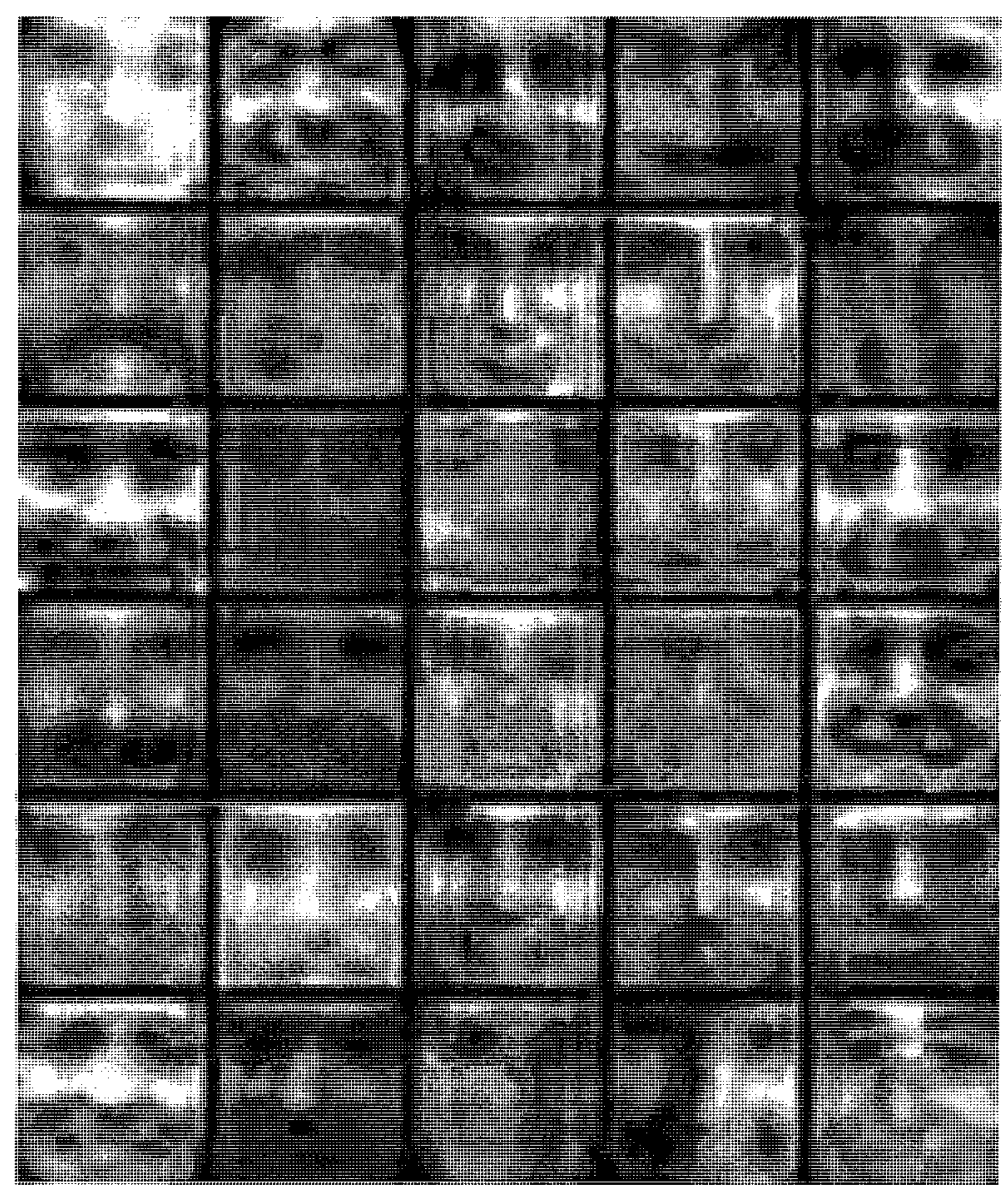

Figure A.5: face images of MIT CBCL training set [20] 


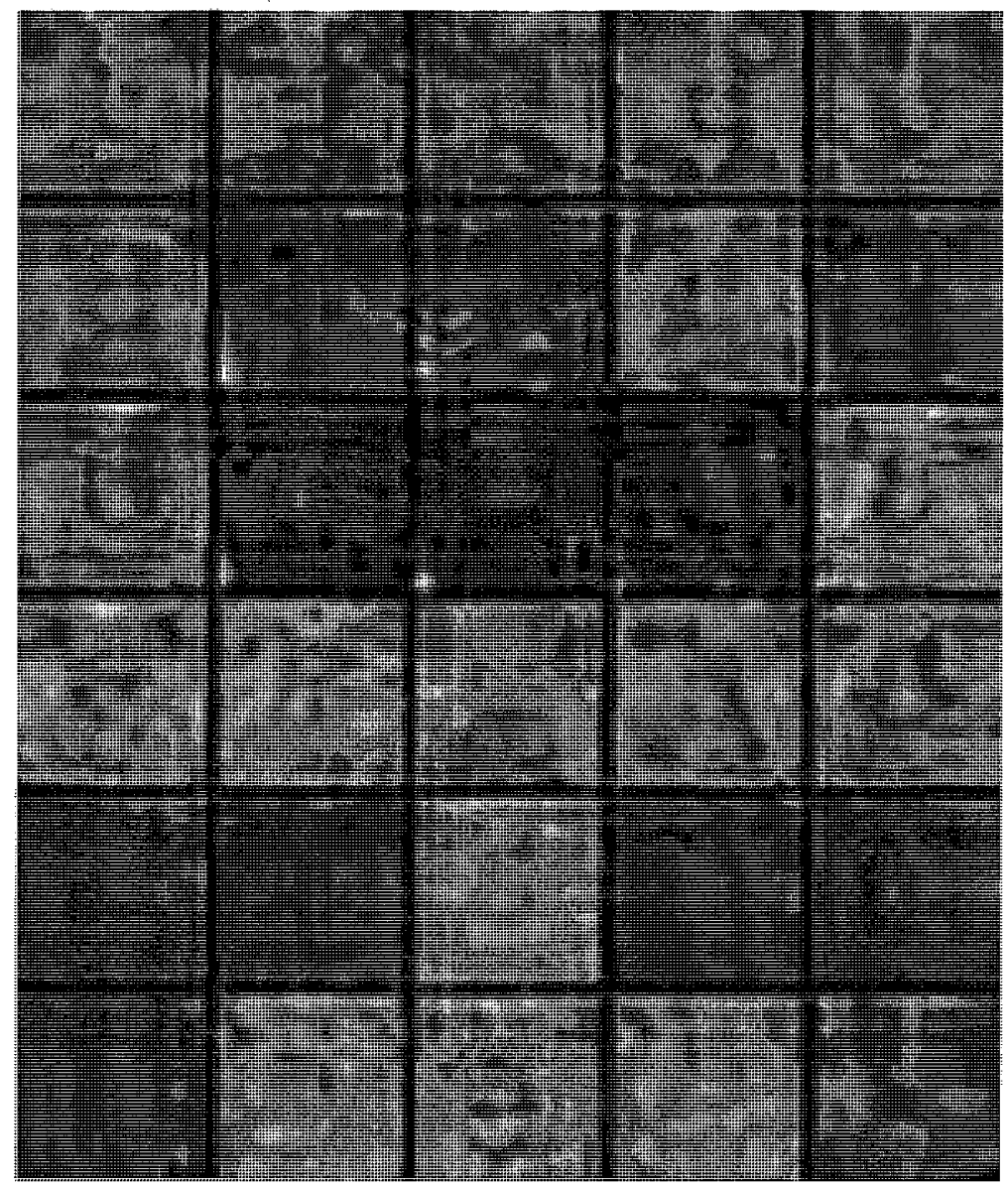

Figure A.6: non-face images of our data set1 at scramble level 22 


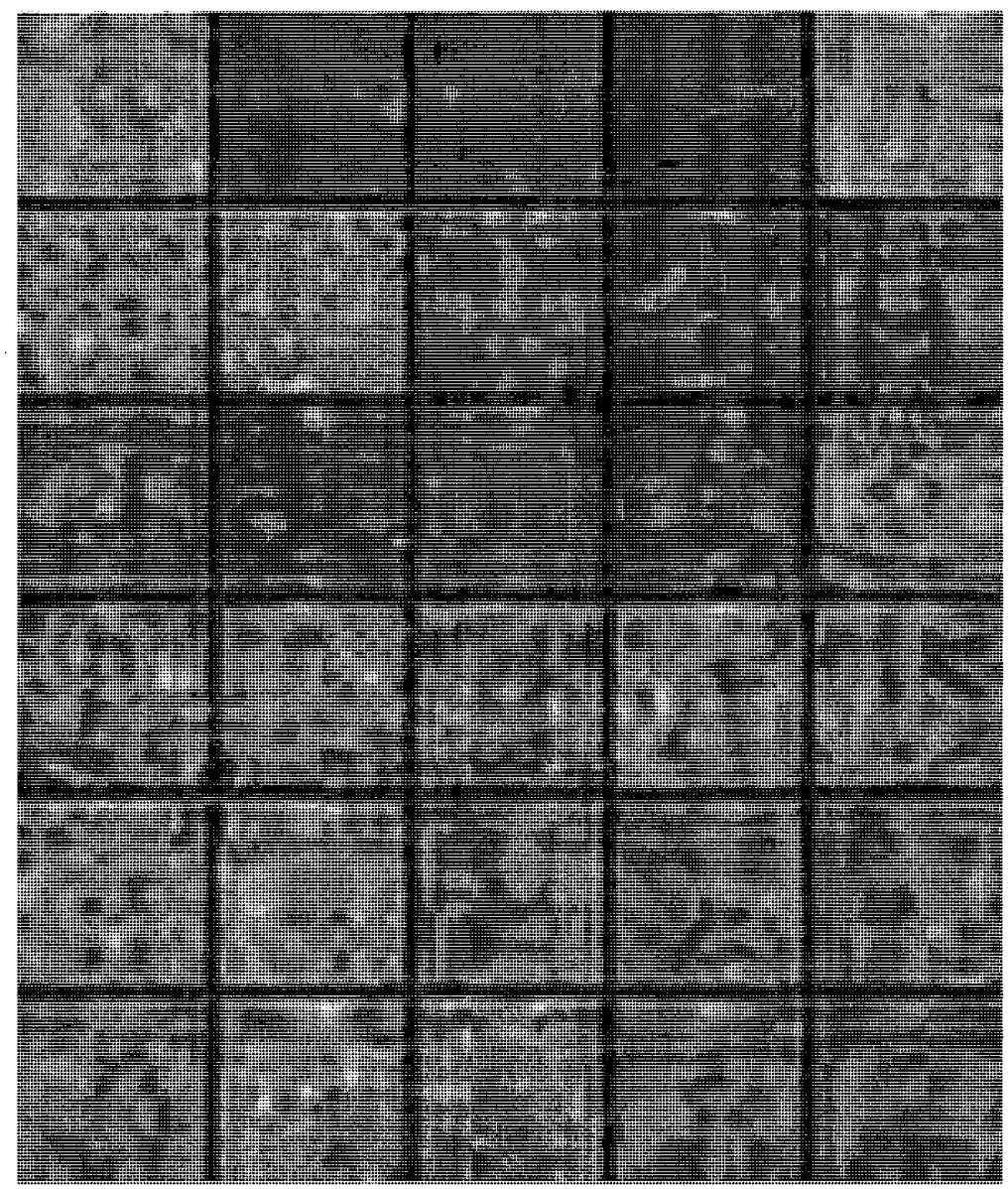

Figure A.7: non-face images of our data set1 at scramble level 28 


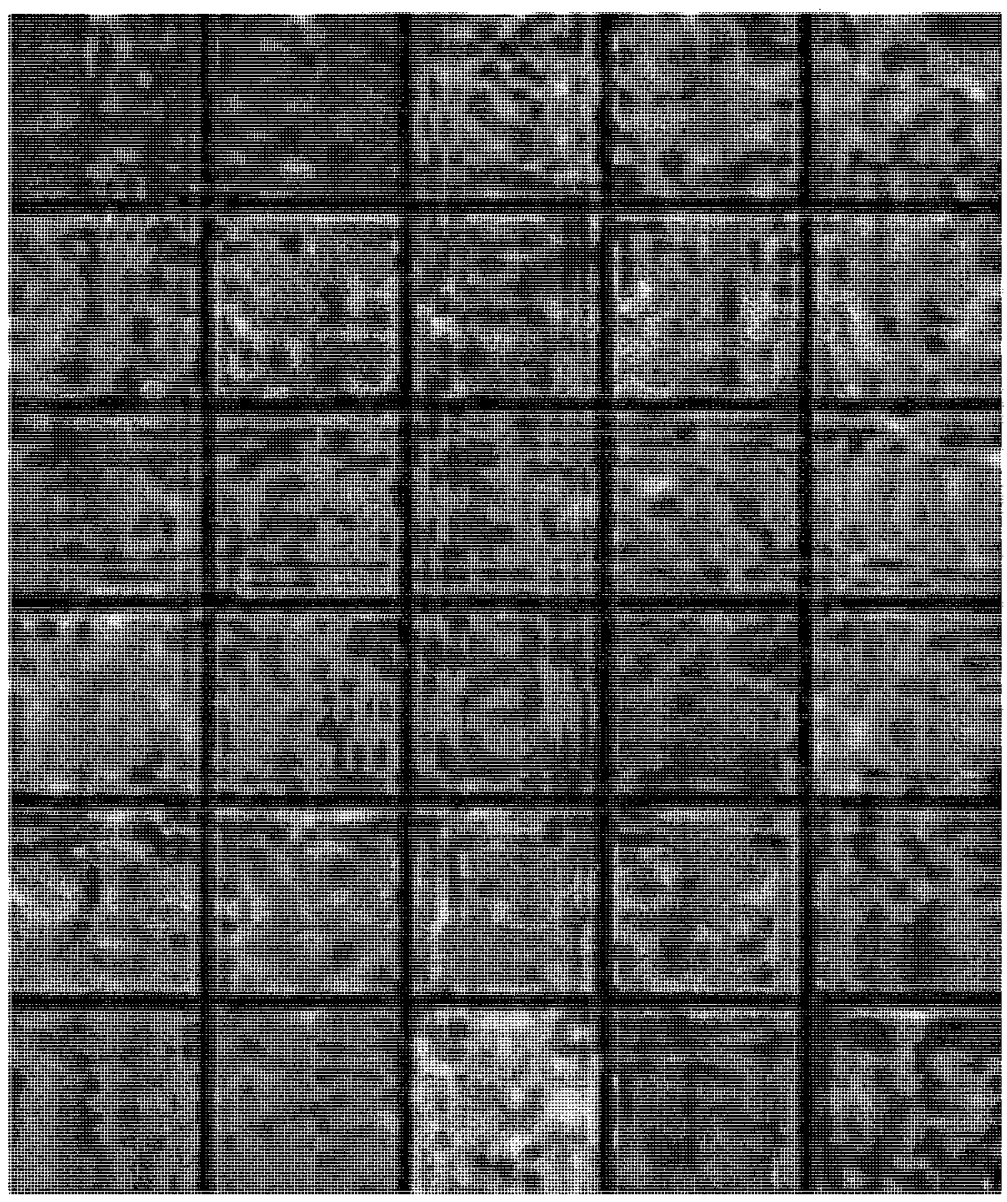

Figure A.8: non-face images of our data set1 at scramble level 34 


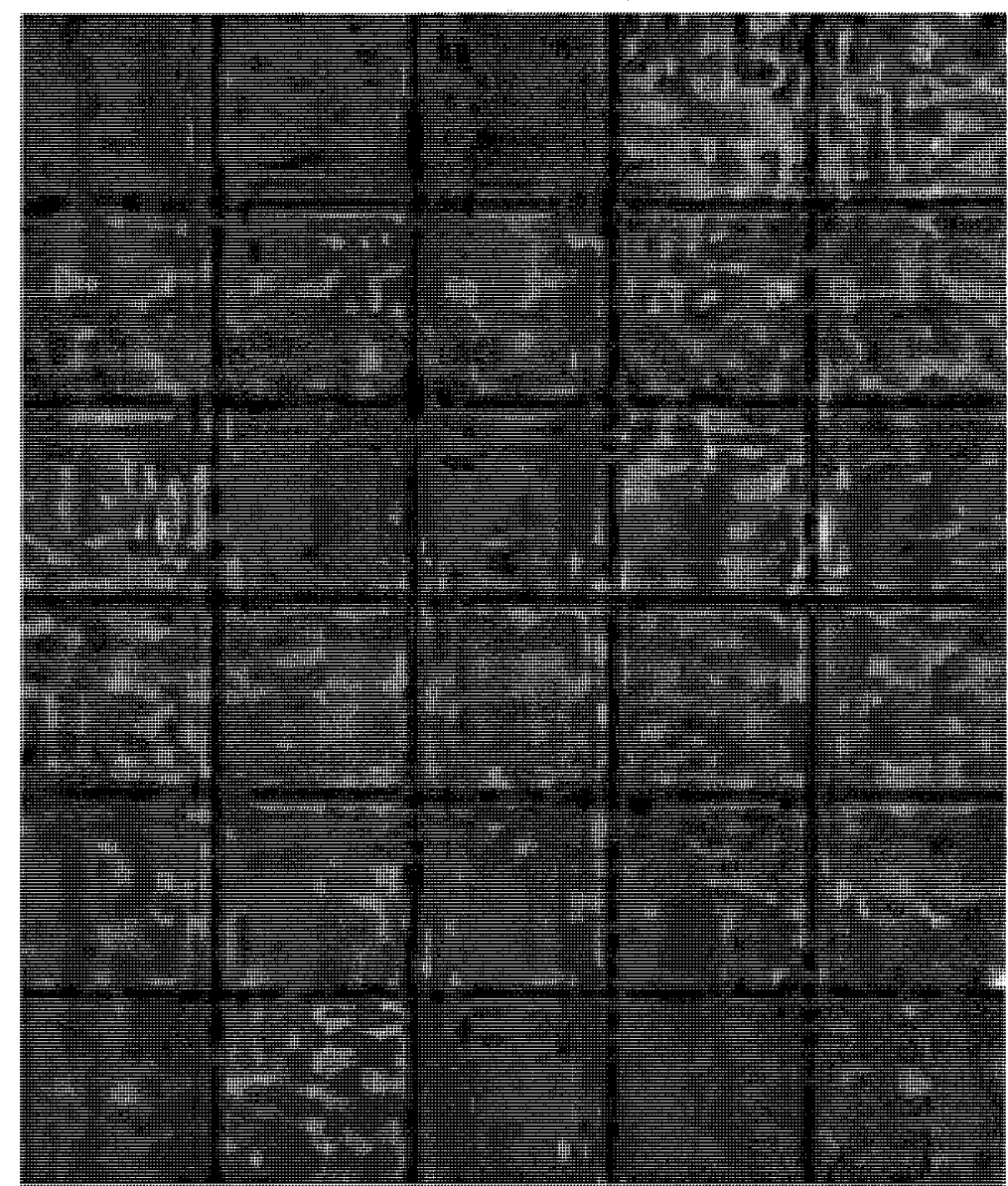

Figure A.9: non-face images of our data set1 at scramble level 40 


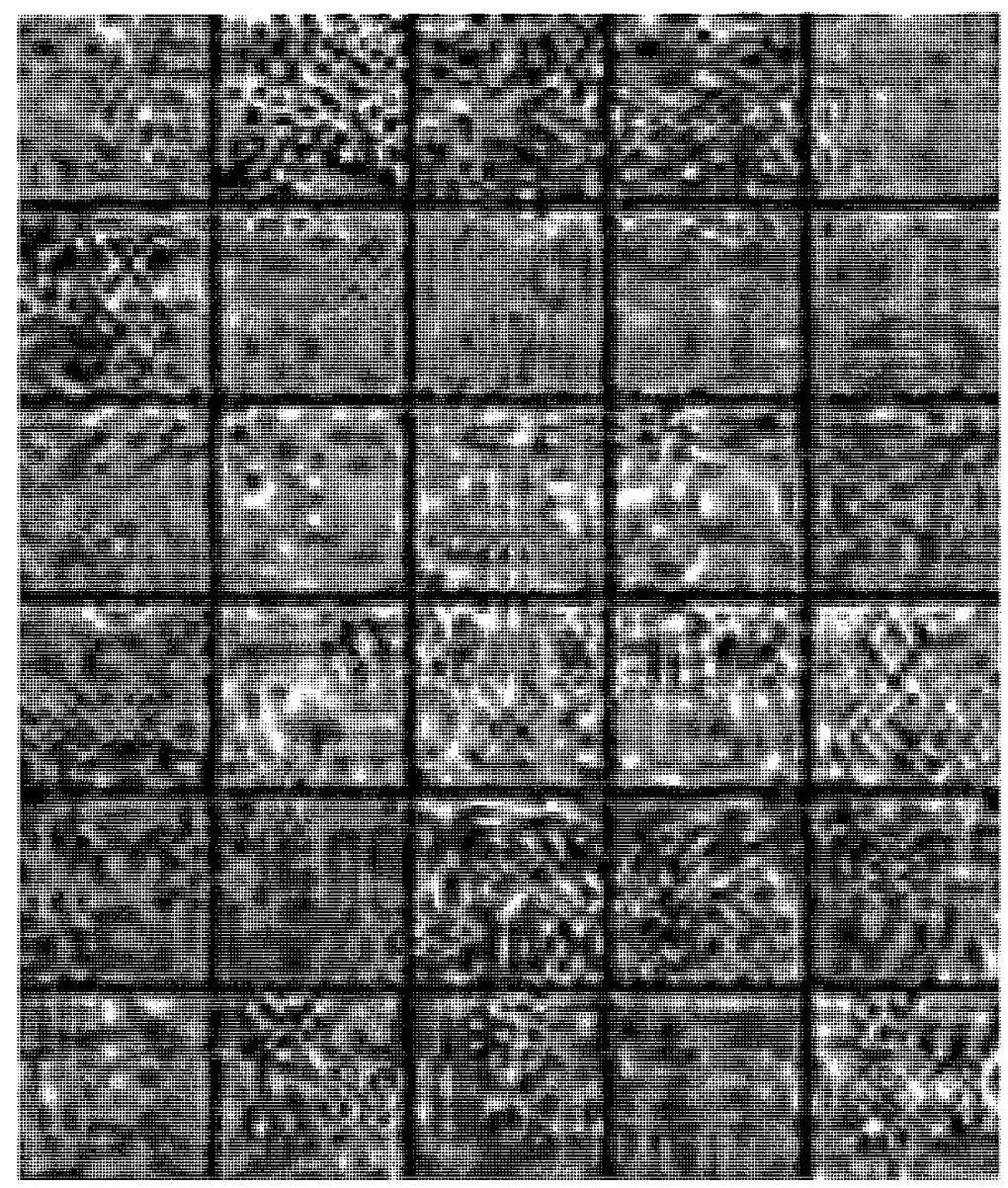

Figure A.10: non-face images of our data set1 at scramble level 46 


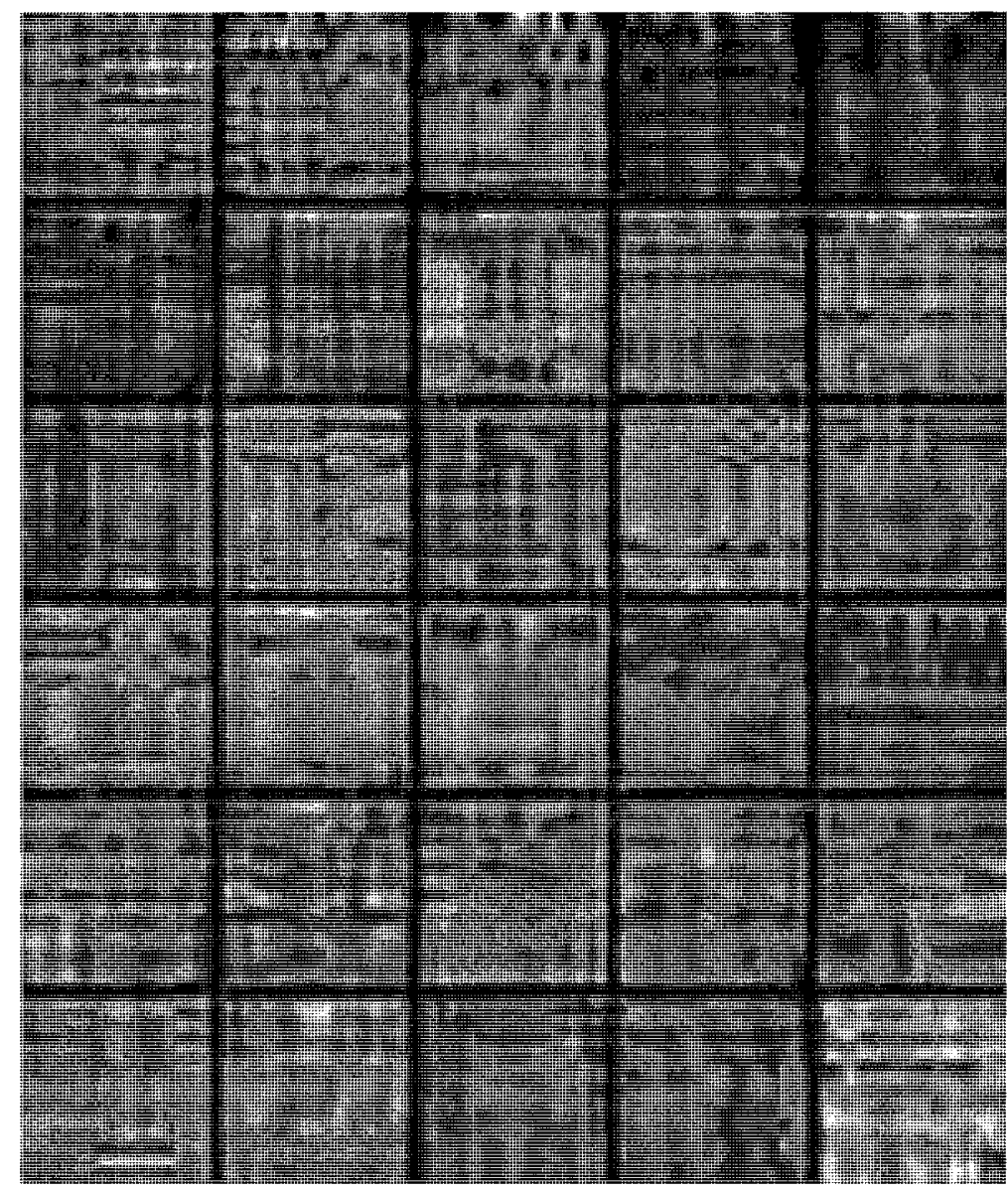

Figure A.11: non-face images of our data set2 at scramble level 8 


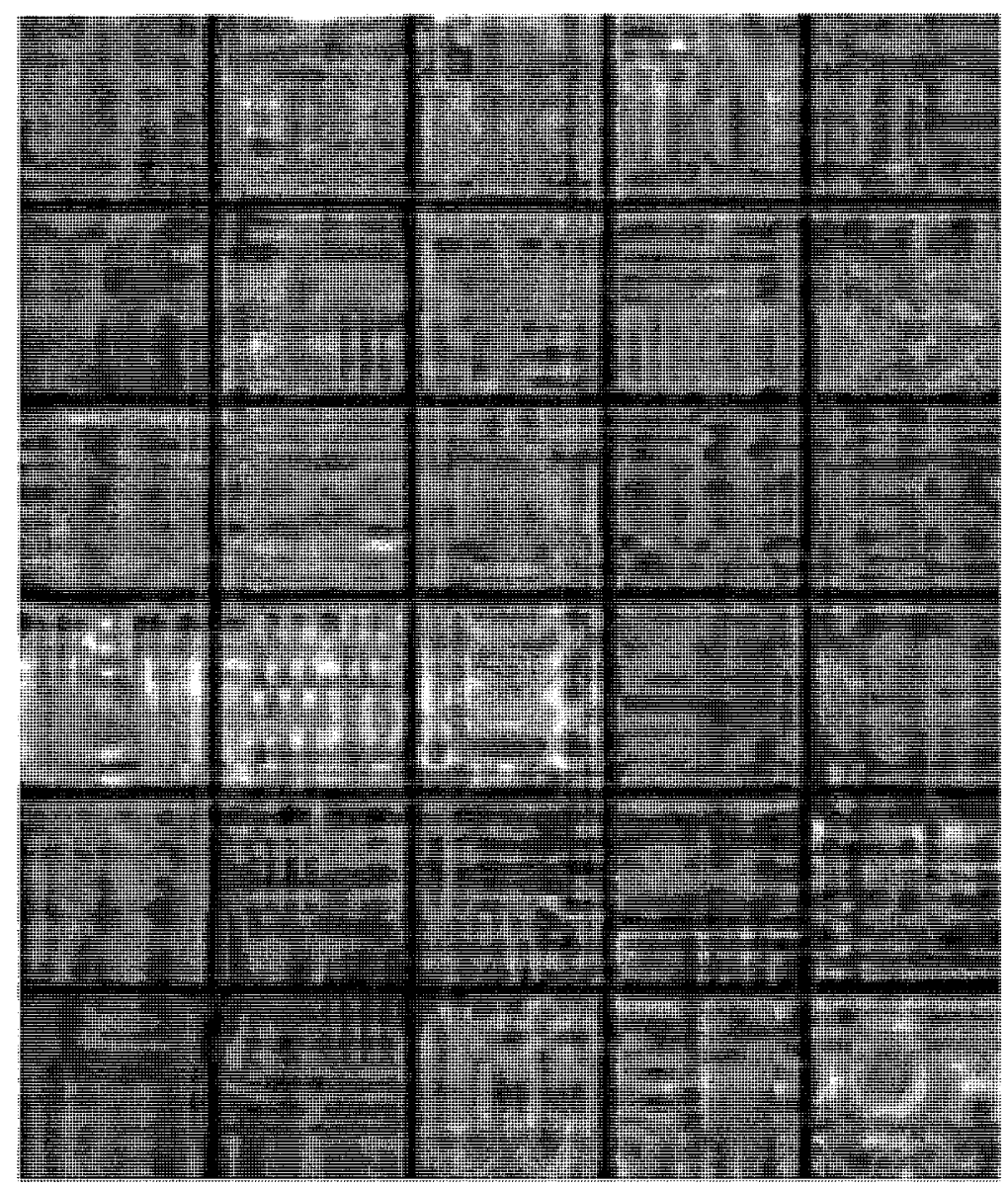

Figure A.12: non-face images of our data set2 at scramble level 10 


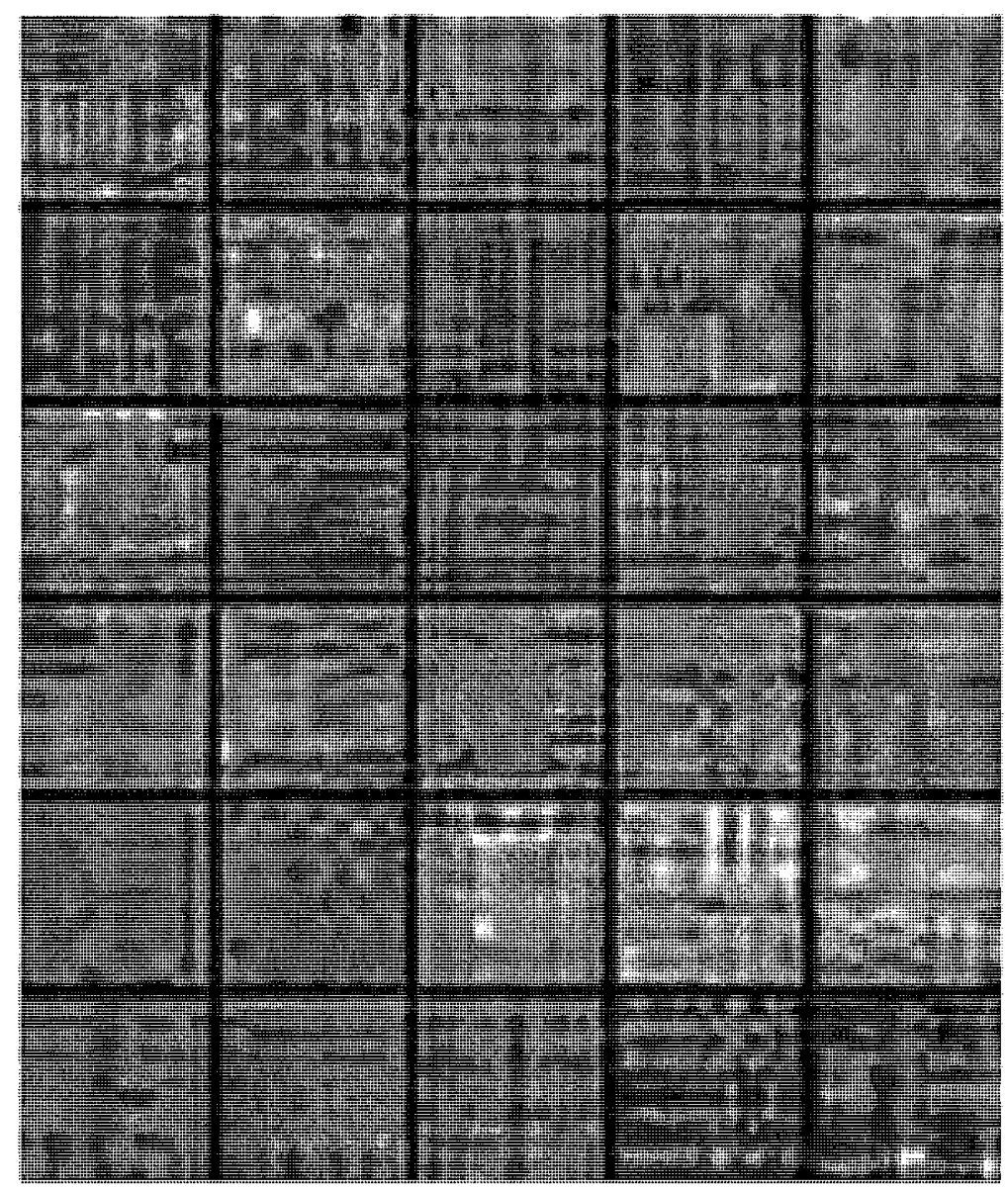

Figure A.13: non-face images of our data set2 at scramble level 13 


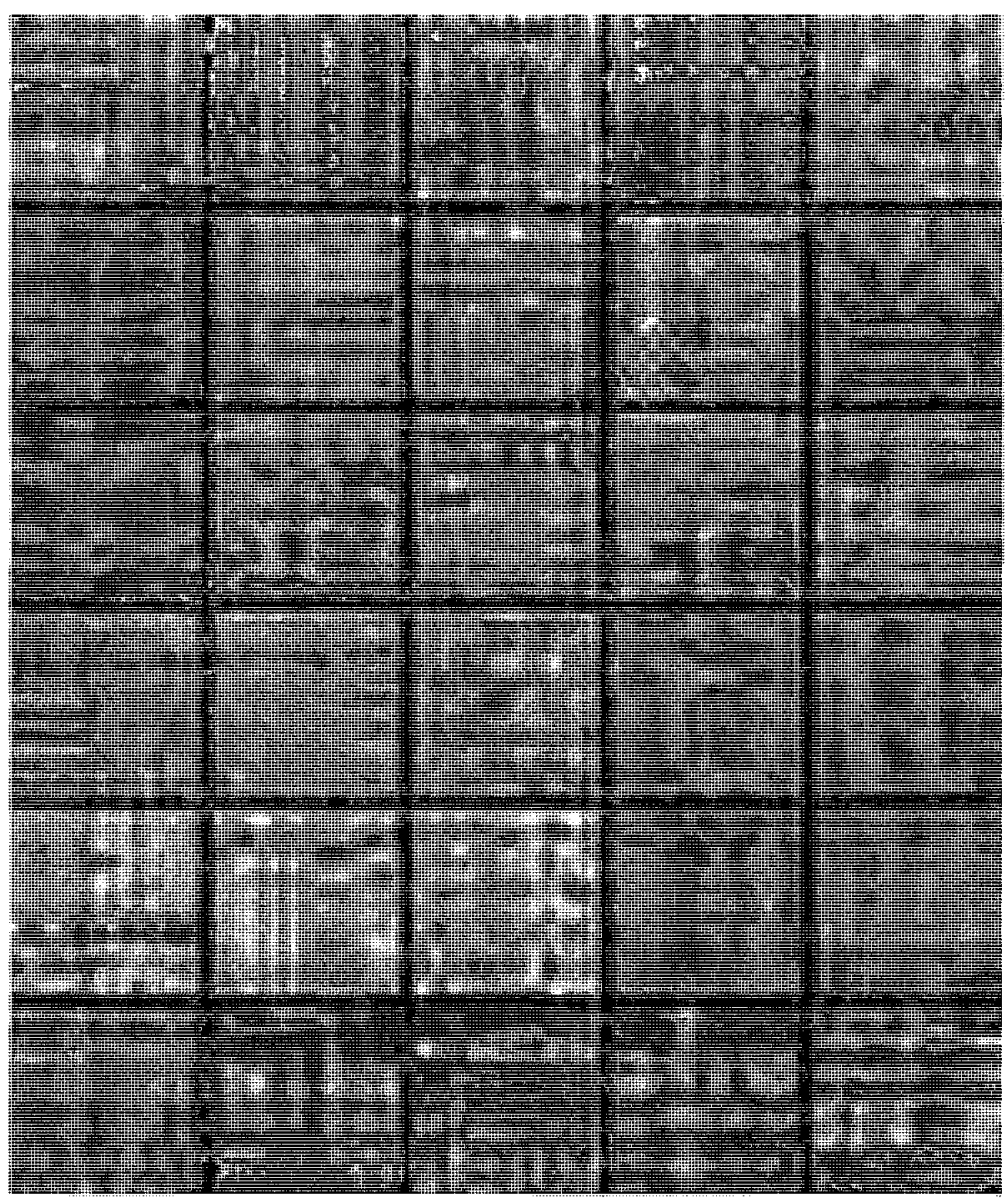

Figure A.14: non-face images of our data set2 at scramble level 17 


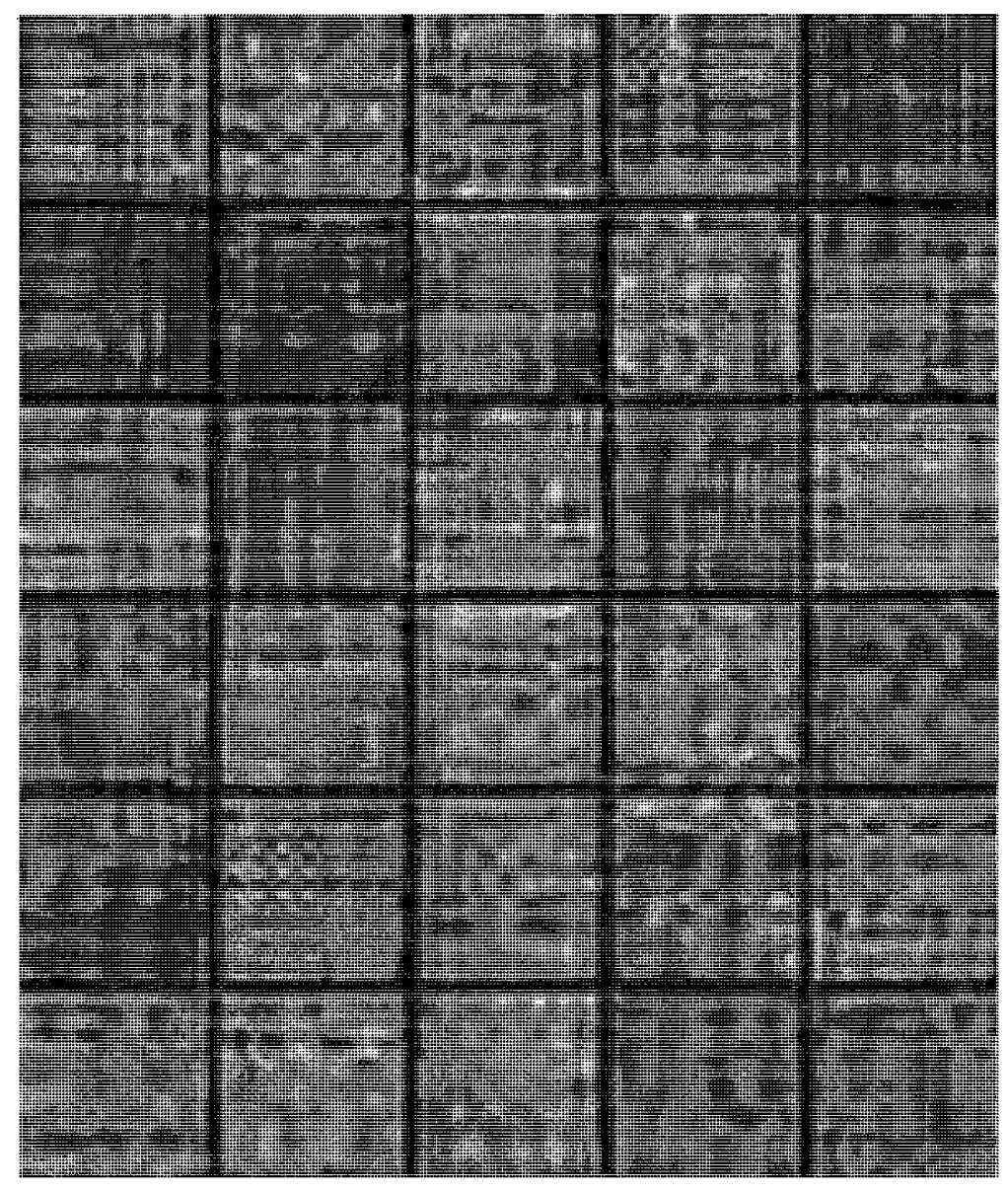

Figure A.15: non-face images of our data set2 at scramble level 20 